\title{
Design, synthesis and biological evaluation of photoaffinity probes of antiangiogenic homoisoflavonoids
}

\author{
Bit Lee ${ }^{a}$, Wei Sun ${ }^{a}$, Hyungjun Lee ${ }^{a}$, Halesha Basavarajappa ${ }^{\text {b,c }}$, Rania S. Sulaiman ${ }^{\text {b,de, }}$, Kamakshi Sishtla ${ }^{\text {, }}$ \\ Xiang Fei ${ }^{\mathrm{a}}$, Timothy W. Corson ${ }^{\mathrm{b}, \mathrm{c}, \mathrm{d}}$, and Seung-Yong Seo ${ }^{\mathrm{a}, *}$ \\ ${ }^{a}$ College of Pharmacy and Gachon Institute of Pharmaceutical Sciences, Gachon University, Incheon 21936, South Korea \\ ${ }^{b}$ Eugene and Marilyn Glick Eye Institute, Departmentof Ophthalmology, Indiana University School of Medicine, Indianapolis, Indiana 46202 , United States of \\ America \\ ${ }^{c}$ Department of Biochemistry and Molecular Biology, Indiana University School of Medicine, Indianapolis, Indiana 46202 , United States of America \\ ${ }^{d}$ Department of Pharmacology and Toxicology, Indiana University School of Medicine, Indianapolis, Indiana 46202, United States of America \\ ${ }^{e}$ Department of Biochemistry, Faculty of Pharmacy, Cairo University, Cairo, Egypt
}

\section{ARTICLE INFO}

\section{A BSTRACT}

\section{Article history:}

Received

Revised

Accepted

Available online

\section{Keywords:}

homoisoflavonoids

photoaffinity probes

antiangiogenic agents

human retinal microvascular endothelial cells

wet age-related macular degeneration

\begin{abstract}
Cremastranone (1), a naturally occurring homoisoflavonoid, inhibited angiogenesis in vitro and in vivo. We developed an analogue SH-11037 (2) which is more potent than cremastranone in human retinal microvascular endothelial cells (HRECs) and blocks neovascularization in animal models. Despite its efficacy, the mechanism of this compound is not yet fully known. In the course of building on a strong foundation of SAR and creating a novel chemical tool for target identification of homoisoflavonoid-binding proteins, various types of photoaffinity probes were designed and synthesized in which benzophenone and biotin were attached to homoisoflavanonoids using PEG linkers on either the C-3' or C-7 position. Notably, the photoaffinity probes linking on the phenol group of the C-3' position retain excellent activity of inhibiting retinal endothelial cell proliferation with up to $72 \mathrm{nM}$ of $\mathrm{GI}_{50}$.
\end{abstract}

2016 Elsevier Ltd. All rights reserved.
The identification of target proteins binding bioactive small molecules is an essential task in drug development that can extend the understanding of mechanisms and provide information for designing new drug candidates. ${ }^{1-9}$ Among many approaches to identify cellular targets of bioactive molecules, biochemical affinity purification using synthetic affinity probes that mimic the structure of the bioactive compounds is a straightforward and powerful method for identifying target molecules. Recently, photoaffinity labeling has been considered a more efficient variant of this method, as it can enrich for low-abundance or transiently-interacting targets. ${ }^{10-14}$ So far, there have been a number of successful examples that have determined the target molecules and identified the binding site through the formation of a covalent bond between the ligand and the specific protein. ${ }^{15-}$ 23 In general, photoaffinity probes contain three functional groups: a bioactive scaffold, a photoreactive group and a reporter tag. ${ }^{24} \mathrm{~A}$ biotin tag is widely employed as a reporter tag because it binds streptavidin or avidin with extremely high affinity.

Cremastranone (1), a naturally occurring homoisoflavanone is reported to have antiangiogenic activity with some selectivity for blocking proliferation of human retinal endothelial cells over other ocular cell types. ${ }^{25-28}$ It inhibits ocular angiogenesis, without substantial toxicity, in mouse models of laser-induced choroidal neovascularization (L-CNV) and oxygen-induced retinopathy (OIR). These animal models recapitulate features of the blinding eye diseases wet age-related macular degeneration and retinopathy of prematurity, respectively. Cremastranone impinges on multiple signaling pathways. It induces expression of p21WAF1 (CDKN1A), which inhibits the cyclin-dependent kinase Cdc2 (CDK1) ${ }^{29}$ It also blocks prostaglandin synthes is and decreases activation of the mitogen activated protein kinases. ${ }^{30,31}$ Finally, it blocks nuclear trans location of NF- $\mathrm{KB}$ and production of inflammatory cytokines. ${ }^{25}, 31,32$ Seeking more potent and selective compounds than cremastranone, we identified $\mathrm{SH}$ 11037 which is observed to have novel antiangiogenic activity against human retinal microvascular endothelial cells (HRECs) and blocks neovascularization in the OIR and L-CNV models. ${ }^{27}$, ${ }^{33}$ Although the studies of mechanisms and medicinal chemistry could contribute to many therapeutic facets of the antiangiogenic homois oflavonoids, their exact mechanis ms of action and cellular targets remain unknown, so the search for direct interacting proteins is valuable. ${ }^{34,35}$ Herein we report design and synthes is of homois oflavonoid-biotin conjugates for inhibiting proliferation

* Corresponding author. Tel.: +82-32-820-4949; fax: +82-32-820-4829; e-mail: syseo@ gachon.ac.kr

This is the author's manuscript of the article published in final edited form as:

Lee B, Sun W, Lee H, Basavarajappa HD, Sulaiman RS, Sishtla K, Fei X, Corson TW, Seo SY. 2016. Design, synthesis and biological evaluation of photoaffinity probes of antiangiogenic homoisoflavonoids. Bioorganic \& Medicinal Chemistry Letters, http://dx.doi.org/10.1016/j.bmcl.2016.07.043 
<smiles>COc1ccc(CC2COc3cc(O)c(O)c(O)c3C2=O)cc1O</smiles><smiles>COc1ccc(CC2COc3cc(OC)c(OC)c(OC)c3C2=O)cc1OC(=O)[C@H](Cc1ccc(C2CC2)cc1)NC(=O)OCc1ccccc1</smiles>

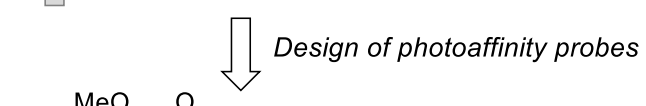

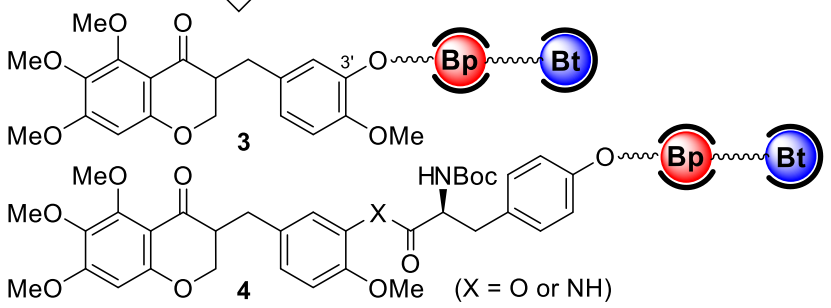<smiles></smiles>

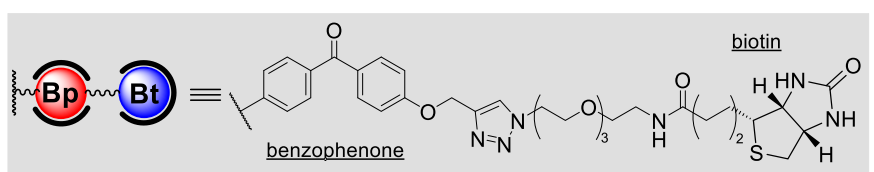

Figure 1. Structures of cremastranone (1), homoisoflavanone analogue SH11037 (2) and photoaffinity probes (3 5). Bp: benzophenone, Bt: biotin.

of endothelial cells and identifying targets, adapting a synthetic strategy we previously employed to generate homoisoflavanones.

On the basis of our SAR investigations in which the A and Bring modifications of homoisoflavonoids with trimethoxy and Boc-phenylalanyl groups, respectively, had the most beneficial effects on its activities and selectivities, trifunctional photoaffinity probes were designed. We synthesized three kinds of photoaffinity probes $(\mathbf{3}-5)$ by linking a trimethoxy derivative of cremastranone and peptidylated active analog SH-11037 (2) to benzophenone and biotin (Figure 1) plus a control compound which lacks the homoisoflavanone moiety, for the future goal of detecting proteins that interact with the homoisoflavonoidlabeled photoaffinity probes in cell lysates. In particular, we modified the homoisoflavanone scaffold at both C-7 and C-3' position with a benzophenone photoreactive group for crosslinking and a biotin reporter group through polyethylene glycol (PEG) as a linker. Thus, it was initially considered to prepare a photoaffinity reagent linking on the phenolic $\mathrm{OH}$ group of the C-3' position. It has been noted that the selected substituents such as $N$-Boc phenylalanyl moiety, which is added to the C-3' position of homoisoflavanone, are essential for the improved antiangiogenic effect. However, because it is uncertain where a linker might impinge on function, we anticipated that SH-11037 (2) could also be modified at the para-position of the phenylalanyl group as well as C-7 without a significant loss of activity by elongating a tethered biotin moiety with hydrophilic groups. Unfortunately, the introduction of a tether to the site of the NHBoc group in SH-11037 (2) was proven to be problematic due to the low reactivity of the amino group and instability of the ester group in the course of Boc-deprotection or linking with a carbamate.

The synthes is of intermediate $\mathbf{1 0}$ is outlined in Scheme 1.24,36 Similar to the procedures reported previously, the synthes is of biotinylated benzophenone was commenced with two different alkylations of 4,4'-dihydroxybenzophenone, which was treated with propargylic bromide and $\mathrm{K}_{2} \mathrm{CO}_{3}$, followed by DIADmediated Mitsunobu reaction using 3-(Boc-amino)-1-propanol to afford the resulting bis-ether 7 . The azide $\mathbf{8}$ which was obtained from treatment of commercially available $p$-nitrophenyl ester of biotin (Biotin-ONp) with $\mathrm{N}_{3}-\mathrm{PEG}_{3}-\mathrm{CH}_{2} \mathrm{CH}_{2} \mathrm{NH}_{2}$ was coupled with bis-ether 7 by $\mathrm{Cu}$-catalyzed Huisgen 1,3-dipolar cycloaddition, ${ }^{37}$ followed by the treatment of the resulting 1,2,3triazole 9 with TFA to afford benzophenone-linked biotin intermediate $\mathbf{1 0}$ in good yield. In future target identification studies, $\mathbf{1 0}$ can also be utilized as a negative control compound which lacks the homois oflavanone moiety.<smiles>C#CCOc1ccc(C(=O)c2ccc(OCCCNC(=O)OC(C)(C)C)cc2)cc1</smiles><smiles>O=C(CCCCC1SCC2NC(=O)NC21)NCCOCCNCCOOCCCO</smiles><smiles>[R]CCCOc1ccc(C(=O)c2ccc(OCc3cn(CCOCCNC(=O)CCC[C@@H]4SC[C@H]5NC(=O)N[C@@H]54)nn3)cc2)cc1</smiles>

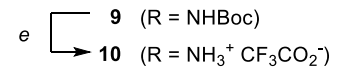

Scheme 1. Synthesis of benzophenone-linked biotin compound (10). Reagen ts and conditions: a) $\mathrm{K}_{2} \mathrm{CO}_{3}$, propargy lic bromide, acetone, $82 \%$; b) DIAD, $\mathrm{B}$ oc-amino-propanol, $\mathrm{PPh}_{3}, \mathrm{~T} \mathrm{HF}, 70 \%$; c) 11-azido-3,6,9-triox aundecan-1-ami ne, $\mathrm{Pr}_{2} \mathrm{NEt}, \mathrm{CH}_{2} \mathrm{Cl}_{2}, 49 \%$; d) $\mathrm{CuSO}_{4} \cdot 5 \mathrm{H}_{2} \mathrm{O}$, sodium ascorbate, $t-\mathrm{BuOH} / \mathrm{H}_{2} \mathrm{O}$. $40 \%$; e) TFA, $\mathrm{CH}_{2} \mathrm{Cl}_{2}, 72 \%$.

To prepare the first photoaffinity probe $\mathbf{3}$, the bioactive and racemic 3-benzyl-4-chromanone $( \pm)-\mathbf{1 1}$ was chosen as a starting material (Scheme 2). ${ }^{25-27}$ The phenolic $\mathrm{OH}$ on the C-3' position<smiles>COc1ccc(CC2COc3cc(OC)c(OC)c(OC)c3C(=O)C2OC)cc1COC(=O)COC(=O)COc1cc(CC2COc3cc(OC)c(OC)c(OC)c3C2=O)ccc1OC</smiles>

$( \pm)-11$

12

13<smiles>CCn1cc(COc2ccc(C(=O)c3ccc(OCCCNC(=O)COc4cc(CC5COc6cc(OC)c(OC)c(OC)c6C5=O)ccc4OC)cc3)cc2)nn1</smiles>

Scheme 2. Synthesis of phot oaffinity probe (3). Reagents and conditions: a) $t$-butyl bromoacetate, $\mathrm{K}_{2} \mathrm{CO}_{3}$, acet one, reflux, $95 \%$; b) $\mathrm{TFA}, \mathrm{CH}_{2} \mathrm{Cl}_{2}$, 70\%; c) 10, $\mathrm{HBTU}, \mathrm{iPr}_{2} \mathrm{NEt}, \mathrm{DMF}, 30 \%$. 
<smiles>COc1ccc(CC2COc3cc(OC)c(OC)c(OC)c3C2=O)cc1CC1COc2cc(OC)c(OC)c(OC)c2C1=O</smiles>

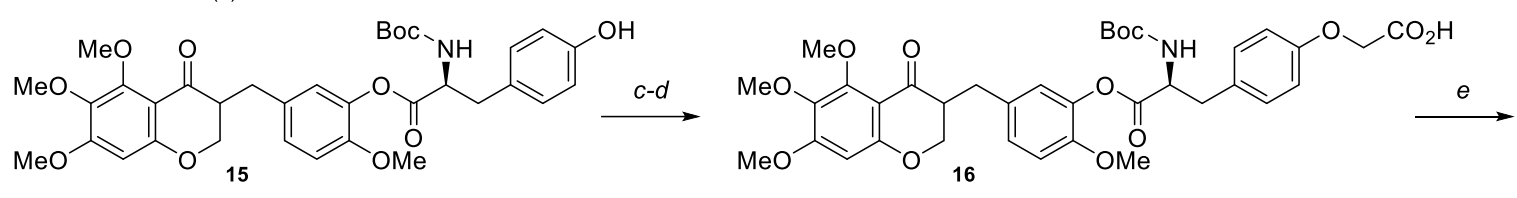

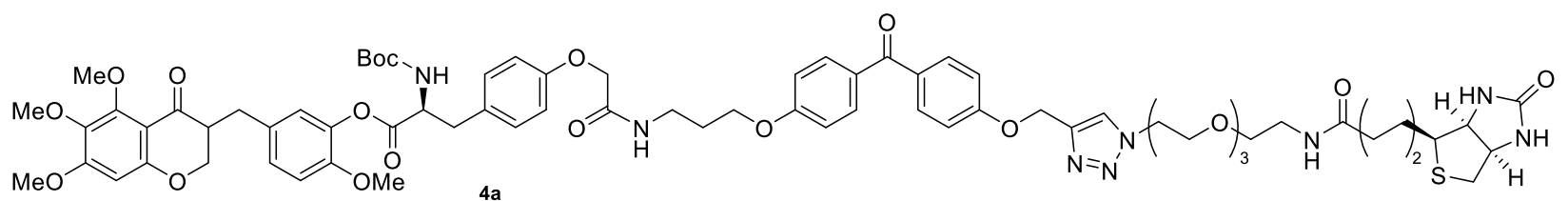

Scheme 3. Synthesis of photoaffinity probe (4a). Reagents and conditions: a) Boc-Tyr(Bn)-OH, EDCI, DMAP, rt, 94\%; b) $\mathrm{H}_{2}, \mathrm{Pd} / \mathrm{C}, \mathrm{EtOAc}, 88 \%$; c) $\mathrm{K}_{2} \mathrm{CO}_{3}$, benzyl bromoacetate, acetone, reflux; d) $\mathrm{H}_{2}, \mathrm{Pd} / \mathrm{C}$, EtOAc, $97 \%$ for 2 steps; e) 10, HBTU, iPr ${ }_{2} \mathrm{NEt}, \mathrm{DMF}, 67 \%$.

was alkylated with tert-butyl bromoacetate under basic conditions, followed by the acidic treatment of the ester $\mathbf{1 2}$ to afford the carboxylic acid 13. Finally, the carboxylic acid $\mathbf{1 3}$ was coupled with the intermediate $\mathbf{1 0}$ by HBTU-mediated amidation to provide the desired photoaffinity probe $\mathbf{3}$ in moderate yield.

For the photoaffinity probes modified at the para-position of the phenylalanyl group of 2, Boc-Tyr(Bn)-OH was introduced into compound $\mathbf{1 1}$ at the C-3' position by using EDCI (Scheme 3). The debenzylation of the ester $\mathbf{1 4}$ afforded the resulting compound 15, which has a free $\mathrm{OH}$ group. The alkylation of $\mathbf{1 5}$ with benzyl bromoacetate and subsequent hydrogenolys is under $\mathrm{H}_{2}$ and $\mathrm{Pd} / \mathrm{C}$ generated the carboxylic acid 16. In the end, the carboxylic acid $\mathbf{1 6}$ was coupled with the intermediate $\mathbf{1 0}$ by HBTU-mediated amidation to afford the desired photoaffinity probe $\mathbf{4 a}$ in good yield.

We next investigated the synthes is of an amide-containing photoaffinity probe, which might have increased stability compared to the ester probe $\mathbf{4 a}$. This amide compound $\mathbf{4 b}$ was synthesized from homoisoflavanone $\mathbf{1 7}^{27}$ having an amino group on the C3' position to which $\mathrm{Boc}-\mathrm{Ty}(\mathrm{Bn})-\mathrm{OH}$ was added by EDCI-mediated amidation (Scheme 4). The resulting amide $\mathbf{1 8}$ was treated with $\mathrm{H}_{2}$ and $\mathrm{Pd} / \mathrm{C}$ to generate phenol 19. With phenol 19 in hand, the alkylation with benzyl 4-bromobutyrate followed by debenzylation provided the carboxylic acid 20. Finally, the carboxylic acid $\mathbf{2 0}$ was coupled with the intermediate $\mathbf{1 0}$ by HBTU-mediated amidation to afford the desired photoaffinity probe $\mathbf{4 b}$ in good yield.

The linking site of the final photoaffinity probe was different from that of the previous probes. Thus benzophenone-linked biotin could be attached at the C-7 position of 2 using our synthetic approach to cremastranone (Scheme 5). According to the procedures reported previously, ${ }^{27}$ the known acetophenone $\mathbf{2 1}$ as a starting material was converted to 7-hydroxy-5,6-dimethoxy4-chromanone 22 via 2 steps in good yield. The C-7 alkylation of the compound 22 with benzyl 4-bromobutyrate provided the compound 23. Subsequently, the aldol condensation of 23 with is ovanillin under acidic conditions gave the unstable 3benzylidene-4-chromanone 24. EDCI-mediated esterification of 24 with Boc-Phe-OH and the catalytic hydrogenation reaction using $\mathrm{H}_{2}, \mathrm{Pd} / \mathrm{C}$ resulted in the removal of the benzyl group and the saturation of the double bond to give the desired homois oflavanone $\mathbf{2 5}$ having butyric acid at the C-7 position. The acid 25 was coupled with the intermediate $\mathbf{1 0}$ by HBTUmediated amidation to afford the final photoaffinity probe $\mathbf{5}$ in moderate yield.

It is obviously important that the synthesized probes retain potency in biological assays. Therefore, we tested the antiproliferative activity of the probes as well as intermediates on human retinal microvascular endothelial cells (HRECs) and human umbilical vein endothelial cells (HUVECs). We also measured the endothelial cell specificity of these compounds by measuring their effects on the proliferation of non-endothelial ocular cell lines, Y79 (retinoblastoma cell line), 92-1 (uveal melanoma cell line) and ARPE19 (retinal pigment epithelium cell line). The results are summarized in Table 1. Among newly synthesized probes, 3 exhibited excellent inhibitory activity with $\mathrm{GI}_{50}$ value of $72 \mathrm{nM}$ against HRECs in spite of the incorporation<smiles>COc1ccc(CC2COc3cc(OC)c(OC)c(OC)c3C2=O)cc1CC1COc2cc(OC)c(OC)c(c2OC)C1=O</smiles><smiles>COc1ccc(CC2COc3cc(OC)c(OC)c(OC)c3C2=O)cc1NC(=O)C(Cc1ccc(O)cc1)NC(=O)c1ccccc1</smiles><smiles>COc1ccc(CC2COc3cc(OC)c(OC)c(OC)c3C2=O)cc1NC(=O)[C@H](Cc1ccc(OC(C)(C)C)cc1)NC(=O)O</smiles>

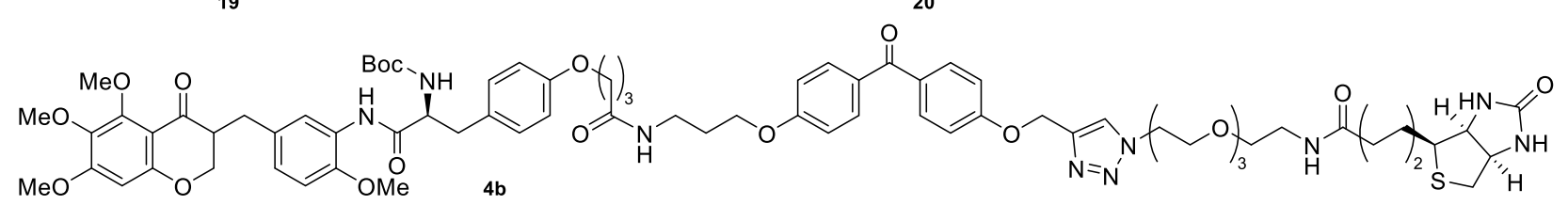

Scheme 4. Synthesis of photoaffinity probe (4b). Reagents and conditions: a) Boc-Tyr(Bn)-OH, EDCI, DMAP, rt, 72\%; b) $\mathrm{H}_{2}, \mathrm{Pd} / \mathrm{C}, \mathrm{EtOAc}, 99 \%$; c) $\mathrm{K}_{2} \mathrm{CO}_{3}$, benzyl 4-bromobut anoate, acetone, reflux; d) $\mathrm{H}_{2}$, Pd/C, EtOAc, $73 \%$ for 2 steps; e) 10, HBTU, iPr $2 \mathrm{NEt}$, DMF, $86 \%$. 

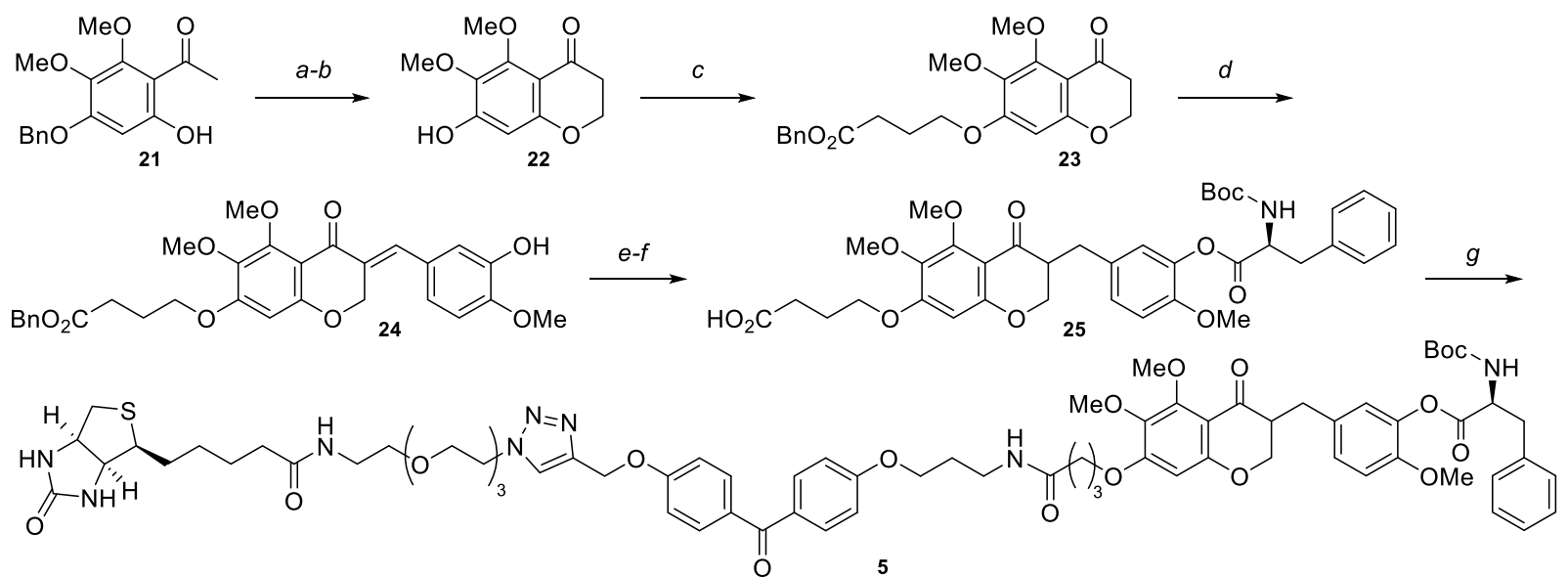

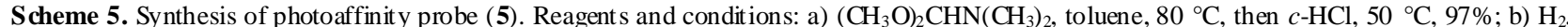
$\mathrm{Pd} / \mathrm{C}, \mathrm{MeOH}, 99 \%$; c) $\mathrm{K}_{2} \mathrm{CO}_{3}$, benzyl 4-bromobutanoate, acetone, reflux, 80\%; d) isovanillin, $p$-TsOH, benzene, reflux, 54\%; e) Boc-Phe-OH, EDCI, DMAP, rt; f) $\mathrm{H}_{2}, \mathrm{Pd} / \mathrm{C}$, EtOAc, $70 \%$ for 2 steps; g) 10, HBTU, iPr 2 NEt, DMF, $36 \%$

of the benzophenone photoreactive group and biotin reporter group. The probe $\mathbf{4 a}\left(\mathrm{GI}_{50}=210 \mathrm{nM}\right)$ also retained potency comparable to cremastranone (1) and SH-11037 (2), but lost some specificity. In contrast, amide probe $\mathbf{4 b}$ and $\mathrm{C}-7$ linking probe $\mathbf{5}$ had somewhat reduced activity on HREC cell proliferation. Therefore, the probes $\mathbf{3}$ and $\mathbf{4 a}$ would be very promising tools for target identification of antiangiogenic homoisoflavonoid-binding proteins.

Table 1. Growth inhibitory activity $\left(\mathrm{GI}_{50}, \mu \mathrm{M}\right)$ of photoaffinity probes and their intermediates on the proliferation of microvascular (HREC), macrovascular (HUVEC) and other ocular (92-1, Y79 and ARPE19) cells.

\begin{tabular}{|c|c|c|c|c|c|}
\hline compd & HREC & HUVEC & $92-1$ & Y79 & ARPE19 \\
\hline $\mathbf{1}^{\mathrm{a}}$ & $\begin{array}{l}0.22 \\
(0.12- \\
0.39)^{\mathrm{b}}\end{array}$ & $\begin{array}{l}0.38 \\
(0.24- \\
0.59)\end{array}$ & $\begin{array}{l}48 \\
(17-132)\end{array}$ & $\begin{array}{l}9.8 \\
(2.1-45)\end{array}$ & $\mathrm{ND}^{\mathrm{c}}$ \\
\hline $2^{a}$ & $\begin{array}{l}0.055 \\
(0.032- \\
0.094)\end{array}$ & $\begin{array}{l}0.75 \\
(0.37- \\
1.5)\end{array}$ & $>100$ & $\begin{array}{l}12 \\
(5.7-25)\end{array}$ & ND \\
\hline 3 & $\begin{array}{l}0.072 \\
(0.00035 \\
-0.15)\end{array}$ & $>100$ & $>100$ & $>100$ & $>100$ \\
\hline $4 a$ & $\begin{array}{l}0.21 \\
(0.041- \\
1.1)\end{array}$ & $\begin{array}{l}0.44 \\
(0.15- \\
1.3)\end{array}$ & $>100$ & $>100$ & $>100$ \\
\hline $4 b$ & $\begin{array}{l}42 \\
(4.1- \\
444)\end{array}$ & $>100$ & $>100$ & $>100$ & $>100$ \\
\hline 5 & $\begin{array}{l}44 \\
(11-171)\end{array}$ & $>100$ & $>100$ & $>100$ & $>100$ \\
\hline $11^{\mathrm{a}}$ & $\begin{array}{l}2 \\
(0.81- \\
5.1)\end{array}$ & $\begin{array}{l}12 \\
(2.7-55)\end{array}$ & $>100$ & $>100$ & ND \\
\hline $14^{\mathrm{a}}$ & $\begin{array}{l}0.51 \\
(0.26- \\
1.0)\end{array}$ & $>100$ & $>100$ & $>100$ & ND \\
\hline 16 & $\begin{array}{l}0.047 \\
(0.0062- \\
0.36)\end{array}$ & $\begin{array}{l}0.012 \\
(0.0018- \\
0.084)\end{array}$ & $>100$ & $>100$ & $>100$ \\
\hline $18^{\mathrm{a}}$ & $>100$ & $>100$ & $>100$ & $\begin{array}{l}0.39 \\
(0.12- \\
1.3)\end{array}$ & ND \\
\hline 20 & $\begin{array}{l}0.0079 \\
(0.0018- \\
0.035)\end{array}$ & $\begin{array}{l}22 \\
(3.0- \\
168) \\
\end{array}$ & $>100$ & $>100$ & $>100$ \\
\hline
\end{tabular}

${ }^{a}$ the $\mathrm{GI}_{50}$ values reported in ref $27 .{ }^{\mathrm{b}} 95 \%$ confidence interval shown in parentheses. ${ }^{\mathrm{C} N D}$, not determined.
In conclusion, we have designed and synthesized novel photoaffinity probes of antiangiogenic homoisoflavonoids with photoreactive benzophenone and biotin. In order to search for the photoaffinity probes which retain potency, the attachment of benzophenone and biotin was on $\mathrm{C}^{\prime}$ and $\mathrm{C}-7$ position of homois oflavonoids. Among them, the probes $\mathbf{3}$ and $\mathbf{4 a}$ exhibited potent anti-proliferative and endothelial-cell specific activity when compared with the activity of the natural product cremas tranone and improved analogue SH-11037.

\section{Acknowledgments}

This work was supported by a grant from the Basic Science Research Program through the National Research Foundation of Korea (NRF) funded by the Ministry of Education (NRF2013R1A1A2007151) and the Korea Health Technology R\&D Project through the Korea Health Industry Development Institute (KHIDI), funded by the Ministry of Health \& Welfare (HI14C1135) to S-YS; grants to TWC from the Intemational Retinal Research Foundation, Carl Marshall and Mildred Almen Reeves Foundation, Ralph W. and Grace M. Showalter Research Trust, Retina Research Foundation, NIH NEI (R01EY025641) and NIH NCATS (KL2TR001106); and by an unrestricted grant from Research to Prevent Blindness, Inc..

\section{References and notes}

1. Cong, F.; Cheung, A. K.; Huang, S. M. Annu. Rev. Phamacol. Toxicol. 2012, 52,57.

2. Fut amura, Y.; Muroi, M.; Osada, H. Mol. Biosyst. 2013, 9, 897.

3. Heal, W. P.; Dang, T. H.; Tate, Chem. Soc. Rev. 2011, 40, 246.

4. Lomenick, B.; Olsen, R. W.; Huang, J. ACS Chem. Biol. 2011, 6, 34.

5. Park, J.; Koh, M.; Park, S. B. Mol. Biosyst. 2013, 9, 544.

6. Rix, U.; Superti-Furga, G. Nat. Chem. Biol., 2009, 5, 616.

7. Schenone, M.; Dancik, V.; Wagner, B. K.; Clemons, P. A. Nat. Chem. Biol. 2013, 9, 232.

8. Titov, D. V.; Liu, J. O. Bioorg. Med. Chem. 2012, 20, 1902.

9. Ziegler, S.; Pries, V.; Hedberg, C.; Waldmann, Angew Chem Int Ed Engl, 2013, 52, 2744.

10. Dorman, G.; Prestwich, G. D. Trends Biotechnol. 2000, 18, 64.

11. Hatanaka, Y.; Sadakane, Y. Curr. Top Med. Chem. 2002, 2, 271.

12. Lapinsky, D. J. Bioorg. Med.Chem. 2012, $20,6237$.

13. Li, G.; Liu, Y.; Yu, X.; Li, X. Bioconjug. Chem. 2014, 25, 1172.

14. Smith, E.; Collins, I. Future Med. Chem. 2015, 7, 159.

15. Corson, T.W.; Cavga, H.; Aberle, N.; Crews, C. M. Chembiochem 2011, 12, 1767 . 
16. Kotoku, N.; Nakata, C.; Kawachi, T.; Sato, T.; Guo, X. H.; Ito, A.; Sumii, Y.; Arai, M.; Kobayashi, M. Bioorg. Med.Chem. 2014,22, 2102

17. Lee, K.; Ban, H. S.; Naik, R.; Hong, Y. S.; Son, S.; Kim, B. K.; Xia, Y.; Song, K. B.; Lee, H. S.; Won, M. Angew. Chem. Int. Ed. Engl. 2013, 52, 10286.

18. Mizuhara, T .; Oishi, S.; Ohno, H.; Shimura, K.; Matsuoka, M.; Fujii, N. Bioorg. Med. Chem. 2013, 21, 2079.

19. Naik, R.; Won, M.; Ban,H. S.; Bhattarai, D.; Xu, X.; Eo, Y.; Hong, Y. S.; Singh, S.; Choi, Y.; Ahn, H. C.; Lee, K. J. Med. Chem. 2014, 57,9522.

20. Park, J.; Oh, S.; Park, S. B. Angew. Chem. Int. Ed. Engl. 2012, 51 , 5447.

21. Shi, H.; Zhang, C. J.; Chen, G. Y.; Yao, S. Q. J. Am. Chem. Soc 2012, 134,3001.

22. Suzuki, T.; Okamura, T.; Tomohiro, T.; Iwabuchi, Y.; Kanoh, N. Bioconjug. Chem. 2015,26,389.

23. Zhang, L.; Zhang, Y.; Dong, J.; Liu, J.; Zhang, L.; Sun, H. Bioorg. Med. Chem. Lett. 2012,22, 1036.

24. Trippier, P. C. ChemMedChem 2013, 8, 190

25. Basavarajappa, H. D.; Lee, B.; Fei, X.; Lim, D.; Callaghan, B.; Mund, J. A.; Case, J.; Rajashekhar, G.; Seo, S.-Y.; Corson, T. W. PLoS One 2014, 9, e95694.

26. Lee, B.; Basavarajappa, H. D.; Sulaiman, R. S.; Fei, X.; Seo, S.Y.; Corson, T. W. Org. Biomol. Chem. 2014, 12, 7673.

27. Basavarajappa, H. D.; Lee, B.; Lee, H.; Sulaiman, R. S.; An, H.; Magana, C.; Shadmand, M.; Vayl, A.; Rajashekhar, G.; Kim, E. Y.; Suh, Y. G.; Lee, K.; Seo, S.-Y.; Corson, T.W. J. Med. Chem. $\mathbf{2 0 1 5}, 58,5015$.
28. Shim, J. S.; Kim, J. H.; Lee, J.; Kim, S. N.; Kwon, H. J. Planta Med. 2004, 70, 171

29. Kim, J. H.; Kim, K. H.; Yu, Y. S.; Kim, Y. M.; Kim, K. W.; Kwon, H. J. Biochem. Biophys. Res. Commun. 2007, 362, 848

30. du Toit, K.; Drewes, S. E.; Bodenstein J. Nat. Prod.Res. 2010, 24, 457.

31. Hur, S.; Lee, Y. S.; Yoo, H.; Yang, J. H.; Kim, T. Y. J. Dermatol. Sci. $2010,59,163$.

32. Lee, Y. S.; Hur, S.; Kim, T. Y. Allergy 2014, 69, 453.

33. Sulaiman, R. S.; Merrigan, S.; Quigley, J.; Qi, X.; Lee, B.; Boulton, M. E.; Kennedy, B.; Seo, S.-Y.; Corson, T. W. Sci.Rep 2016, 6, 25509.

34. La Clair, J. J. Nat. Prod. Rep. 2010, 27, 969.

35. Sulaiman, R. S.; Basavarajappa,H. D.; Corson, T. W. Exp. Eye. Res. 2014, 129, 161.

36. Tae, H. S.; Hines, J.; Schneekloth, A. R.; Crews, C. M. Org. Lett. 2010, 12, 4308

37. McKay, C. S.; Finn, M. G. Chem. Biol. 2014, 21, 1075.

\section{Supplementary Material}

Supplementary data associated with this article can be found, in the online version, at doi 
Design, synthesis and biological evaluation of photoaffinity probes of antiangiogenic homoisoflavonoids

Bit Lee, ${ }^{a}$ Wei Sun, ${ }^{a}$ Hyungjun Lee,${ }^{a}$ Halesha Basavarajappa,${ }^{b, c}$ Rania Sulaiman, ${ }^{b, d, e}$ Kamakshi Sishtla, ${ }^{b}$ Xiang $\mathrm{Fei}^{a}$, Timothy W. Corson, ${ }^{b, c, d}$ and Seung-Yong $\mathrm{Seo}^{a, *}$

${ }^{a}$ College of Pharmacy and Gachon Institute of Pharmaceutical Sciences, Gachon University, Incheon 21936, South Korea

${ }^{b}$ Eugene and Marilyn Glick Eye Institute, Department of Ophthalmology, Indiana University School of Medicine, Indianapolis, Indiana 46202, United States of America

${ }^{c}$ Department of Biochemistry and Molecular Biology, Indiana University School of Medicine,

Indianapolis, Indiana 46202, United States of America

${ }^{d}$ Department of Pharmacology and Toxicology, Indiana University School of Medicine, Indianapolis,

Indiana 46202, United States of America

${ }^{e}$ Department of Biochemistry, Faculty of Pharmacy, Cairo University, Cairo, Egypt

\section{Synthetic Methodology}

All starting materials and reagents were obtained from commercial suppliers and were used without further purification. Air and moisture sensitive reactions were performed under an argon atmosphere. Flash column chromatography was performed using silica gel 60 (230-400 mesh, Merck) with the indicated solvents. Thin-layer chromatography was performed using $0.25 \mathrm{~mm}$ silica gel plates (Merck). ${ }^{1} \mathrm{H}$ and ${ }^{13} \mathrm{C}$ NMR spectra were recorded on a Bruker $600 \mathrm{MHz}$ spectrometer as solutions in deuteriochloroform $\left(\mathrm{CDCl}_{3}\right)$ or methanol-d4. ${ }^{1} \mathrm{H}$ NMR data were reported in the order of chemical shift, multiplic ity (s, singlet; $d$, doublet; $t$, triplet; $m$, multiplet and/or multiple resonances), number of protons, and coupling constant $(J)$ in hertz $(\mathrm{Hz})$. High-resolution mass spectra (HRMS) were recorded on a JEOL JMS-700 (FAB and EI) and an Agilent 6530 Q-TOF LC/MS/MS system (ESI).

(4-hydroxyphenyl)(4-(prop-2-yn-1-yloxy)phenyl)methanone (6')<smiles>C#CCOc1ccc(C(=O)c2ccc(O)cc2)cc1</smiles> 
To a solution of 4,4'-dihydroxybenzophone $(500 \mathrm{mg}, 2.33 \mathrm{mmol})$ in acetone $(5.0 \mathrm{ml})$ at $0{ }^{\circ} \mathrm{C}$, $\mathrm{K}_{2} \mathrm{CO}_{3}(161 \mathrm{mg}, 1.17 \mathrm{mmol})$ and propargyl bromide $(0.11 \mathrm{ml}, 1.16 \mathrm{mmol})$ were added. The reaction mixture was stirred at $50{ }^{\circ} \mathrm{C}$ for $3 \mathrm{~h}$, cooled to room temperature, and quenched with $\mathrm{H}_{2} \mathrm{O}(10 \mathrm{~mL})$. The mixture was extracted three times with ethyl acetate. The combined extracts were washed with brine, dried over $\mathrm{MgSO}_{4}$, filtered, and concentrated. The residue was purified via Biotage (ethyl acetate $/ n$-hexane $=1: 2$ Snap Ultra $25 \mathrm{~g}$ column). The desired fractions were collected and concentrated to give propargylated benzophenone (6') (240 mg, 82\% BRSM). ${ }^{1} \mathrm{H}-\mathrm{NMR}$ (600 MHz, $\left.\mathrm{CD}_{3} \mathrm{OD}\right) \delta 7.73(\mathrm{~d}, 2 \mathrm{H}, J=8.4 \mathrm{~Hz}), 7.67(\mathrm{~d}, 2 \mathrm{H}, J=8.4 \mathrm{~Hz}), 7.09$ (d, 2H, $\left.J=9.0 \mathrm{~Hz}\right), 6.87$ (d, 2H, $J=$ $9.0 \mathrm{~Hz}), 4.82(\mathrm{~s}, 2 \mathrm{H}), 3.00(\mathrm{t}, 1 \mathrm{H}, J=2.4 \mathrm{~Hz}) ;{ }^{13} \mathrm{C}-\mathrm{NMR}\left(150 \mathrm{MHz}, \mathrm{CD}_{3} \mathrm{OD}\right) \delta$ 195.4, 162.0, 161.0, 132.3, 131.6, 131.1, 128.9, 114.7, 114.7, 77.8, 75.9, 55.3; HRMS (ESI): mass calcd for $\mathrm{C}_{16} \mathrm{H}_{15} \mathrm{O}_{3}[\mathrm{M}$ $+\mathrm{H}]^{+}, 253.0786$; found, 253.0872 .

tert-Butyl (3-(4-(4-(prop-2-yn-l-yloxy)benzoyl)phenoxy)propyl)carbamate (7)

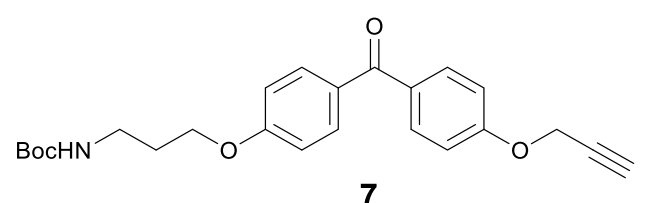

To a THF solution $(2 \mathrm{~mL})$ of $N$-Boc-3-aminopropanol $(117 \mathrm{mg}, 0.76 \mathrm{mmol})$ were added the propargylated benzophenone $\left(6^{\prime}\right)(140 \mathrm{mg}, 0.56 \mathrm{mmol}), \mathrm{PPh}_{3}(150 \mathrm{mg}, 0.76 \mathrm{mmol})$, and DIAD (130 $\mu \mathrm{L}, 0.56 \mathrm{mmol}$ ) at room temperature. After stirring for $3 \mathrm{~h}$ at room temperature, the reaction mixture was diluted with ethyl acetate and the combined organic phase was washed with water and brine, dried over anhydrous $\mathrm{MgSO}_{4}$ and concentrated under reduced pressure. The residue was purified by flash column chromatography on silica gel (ethyl acetate $/ n$-hexane $=1: 3$ ) to afford the dialkylated dihydroxybenzophenone (7) (160 mg, 70\%). ${ }^{1} \mathrm{H}-\mathrm{NMR}\left(600 \mathrm{MHz}, \mathrm{CDCl}_{3}\right) \delta 7.77$ (dd, 4H, $J=9.0$ and $5.0 \mathrm{~Hz}), 7.03(\mathrm{~d}, 2 \mathrm{H}, J=9.0 \mathrm{~Hz}), 6.93(\mathrm{~d}, 2 \mathrm{H}, J=9.0 \mathrm{~Hz}), 4.82(\mathrm{bs}, 1 \mathrm{H}), 4.75(\mathrm{~d}, 2 \mathrm{H}, J=1.2 \mathrm{~Hz})$, $4.09(\mathrm{t}, 2 \mathrm{H}, J=6.0 \mathrm{~Hz}), 3.33(\mathrm{~m}, 2 \mathrm{H}), 2.56(\mathrm{t}, 1 \mathrm{H}, J=2.4 \mathrm{~Hz}), 2.01(\mathrm{~m}, 1 \mathrm{H}), 1.42(\mathrm{~s}, 9 \mathrm{H}), 1.25(\mathrm{~m}$, $1 \mathrm{H}) ;{ }^{13} \mathrm{C}-\mathrm{NMR}\left(150 \mathrm{MHz}, \mathrm{CDCl}_{3}\right) \delta 194.3,162.1,160.6,156.0,132.2,132.1,131.5,130.6,114.3$, 113.9, 77.8, 76.1, 65.9, 55.8, 37.8, 29.5, 28.4, 14.2; HRMS (ESI): mass calcd for $\mathrm{C}_{24} \mathrm{H}_{27} \mathrm{NO}_{5}[\mathrm{M}+\mathrm{H}]$ +, 410.1889; found, 410.1956.

$N$-(2-(2-(2-(2-Azidoethoxy)ethoxy)e thoxy)ethyl)-5-((3aS,4S,6a $R)-2-0 x o h e x a h y d r o-1 H$-thie no[3,4d]imidazol-4-yl)pentanamide (8) 


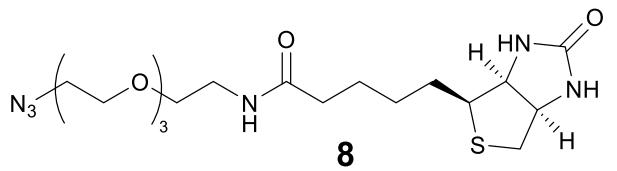

To a THF solution (1.0 mL) of biotin-ONp (50 mg, $0.14 \mathrm{mmol})$ were added 11-azido-3,6,9trioxaundecan-l-amine $(27 \mu \mathrm{L}, 0.14 \mathrm{mmol})$ and $\mathrm{Et}_{3} \mathrm{~N}(57 \mu \mathrm{L}, 0.41 \mathrm{mmol})$ at room temperature. After stirring for $12 \mathrm{~h}$ at room temperature, the reaction mixture was quenched with $\mathrm{H}_{2} \mathrm{O}(1 \mathrm{~mL})$. The reaction mixture was extracted three times with ethyl acetate and the combined organic phase was washed with brine, dried over anhydrous $\mathrm{MgSO}_{4}$, and concentrated under reduced pressure. The residue was purified by flash column chromatography on silica gel ( $\left.\mathrm{MeOH} / \mathrm{CH}_{2} \mathrm{Cl}_{2}=1: 10\right)$ to afford the azide (8) (30 mg, 49\%) ${ }^{1} \mathrm{H}-\mathrm{NMR}\left(600 \mathrm{MHz}, \mathrm{CDCl}_{3}\right) \delta 6.83(\mathrm{~m}, 1 \mathrm{H}), 6.73(\mathrm{~m}, 1 \mathrm{H}), 5.78(\mathrm{~m}$, $1 \mathrm{H}), 4.50(\mathrm{~m}, 1 \mathrm{H}), 4.30(\mathrm{~m}, 1 \mathrm{H}), 3.65(\mathrm{~m}, 8 \mathrm{H}), 3.62(\mathrm{~m}, 2 \mathrm{H}), 3.56(\mathrm{~m}, 2 \mathrm{H}), 3.42-3.37(\mathrm{~m}, 4 \mathrm{H}), 3.13$ $(\mathrm{m}, 1 \mathrm{H}), 2.90(\mathrm{~m}, 1 \mathrm{H}), 2.74(\mathrm{~d}, 1 \mathrm{H}, J=13 \mathrm{~Hz}), 2.22(\mathrm{t}, 2 \mathrm{H}, J=7.8 \mathrm{~Hz}), 1.75-1.62(\mathrm{~m}, 4 \mathrm{H}), 1.44-1.41$ (m, 2H); ${ }^{13} \mathrm{C}-\mathrm{NMR}\left(150 \mathrm{MHz}, \mathrm{CDCl}_{3}\right) \delta 173.4,164.3,70.6,70.4,70.0,70.0,69.9,60.2,55.7,50.6$, 40.5, 39.1, 36.0, 28.3, 28.1, 25.56; HRMS (ESI): mass calcd for $\mathrm{C}_{18} \mathrm{H}_{32} \mathrm{~N}_{6} \mathrm{O}_{5} \mathrm{~S}[\mathrm{M}+\mathrm{H}]^{+}, 445.2155$; found, 445.3135 .

\section{Boc-containing be nzophenone-biotin (9).}

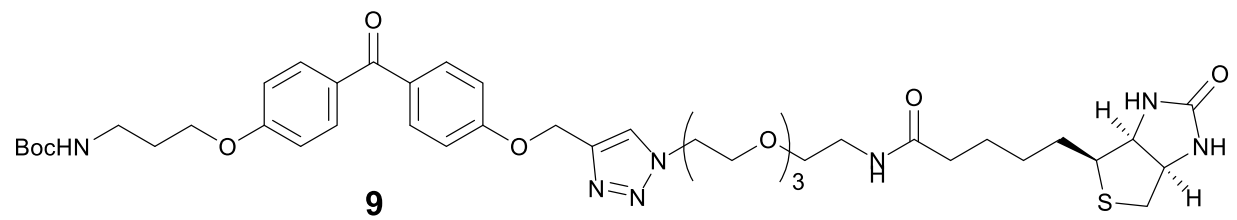

To a $t$ - $\mathrm{BuOH} / \mathrm{H}_{2} \mathrm{O}$ solution $(2 \mathrm{~mL}, 1: 1)$ of the benzophenone (7) $(35 \mathrm{mg}, 0.086 \mathrm{mmol})$ and the PEG-Biotin (8) (38 mg, $0.086 \mathrm{mmol}), \mathrm{CuSO}_{4} \cdot 5 \mathrm{H}_{2} \mathrm{O}(2 \mathrm{mg}, 0.086 \mathrm{mmol})$ and sodium ascorbate (1.0 M in $\mathrm{H}_{2} \mathrm{O}, 2$ drops) were added at room temperature. After stirring at room temperature for $24 \mathrm{~h}$, the reaction mixture was diluted with $\mathrm{H}_{2} \mathrm{O}(1 \mathrm{~mL})$ and extracted with ethyl acetate and the combined organic phase was washed with brine, dried over anhydrous $\mathrm{MgSO}_{4}$, and concentrated under reduced pressure. The residue was purified by flash column chromatography on silica gel $\left(\mathrm{MeOH} / \mathrm{CH}_{2} \mathrm{Cl}_{2}=\right.$ $1: 10)$ to afford the 1,2,3-triazole (30 mg, 40\%). ${ }^{1} \mathrm{H}-\mathrm{NMR}\left(600 \mathrm{MHz}, \mathrm{CDCl}_{3}\right) \delta 7.86(\mathrm{~s}, 1 \mathrm{H}), 7.77$ (dd, $4 \mathrm{H}, J=8.4$ and $2.4 \mathrm{~Hz}$ ), 7.06 (d, 2H, $J=9.0 \mathrm{~Hz}), 6.94$ (d, 2H, $J=8.4 \mathrm{~Hz}), 6.67$ (bs, 1H), 6.45 (bs, 1H), 5.49 (bs, 1H), 5.28 (s, 2H), $4.82(\mathrm{bs}, 1 \mathrm{H}), 4.57$ (t, 2H, J=4.8 Hz), $4.47(\mathrm{~m}, 1 \mathrm{H}), 4.28(\mathrm{~m}, 1 \mathrm{H})$, $4.10(\mathrm{t}, 2 \mathrm{H}, J=6.0 \mathrm{~Hz}), 3.89(\mathrm{t}, 2 \mathrm{H}, J=4.8 \mathrm{~Hz}), 3.65(\mathrm{~m}, 2 \mathrm{H}), 3.60(\mathrm{~m}, 2 \mathrm{H}), 3.56(\mathrm{~m}, 6 \mathrm{H}), 3.52(\mathrm{t}, 2 \mathrm{H}$, $J=4.8 \mathrm{~Hz}), 3.41-3.33(\mathrm{~m}, 4 \mathrm{H}), 3.12(\mathrm{~m}, 1 \mathrm{H}), 2.88(\mathrm{~m}, 1 \mathrm{H}), 2.73(\mathrm{~m}, 1 \mathrm{H}), 2.19(\mathrm{t}, 2 \mathrm{H}, J=12 \mathrm{~Hz})$, 
$2.03(\mathrm{~m}, 2 \mathrm{H}), 1.75-1.58(\mathrm{~m}, 6 \mathrm{H}), 1.43(\mathrm{~s}, 9 \mathrm{H}), 1.41(\mathrm{~m}, 2 \mathrm{H}) ;{ }^{13} \mathrm{C}-\mathrm{NMR}\left(150 \mathrm{MHz}, \mathrm{CDCl}_{3}\right) \delta 194.4$, $173.2,163.9,162.1,161.4,156.0,143.2,132.2,132.2$, 131.2, 130.5, 124.3, 114.3, 113.9, 79.3, 70.6, 70.5, 70.4, 70.3, 70.1, 69.9, 69.3, 65.9, 62.0, 61.7, 60.1, 55.5, 50.6, 50.3, 40.5, 39.1, 37.8, 35.9, 29.5, 28.4, 28.1, 25.5; HRMS (ESI): mass calcd for $\mathrm{C}_{42} \mathrm{H}_{59} \mathrm{~N}_{7} \mathrm{O}_{10} \mathrm{~S}[\mathrm{M}+\mathrm{H}]^{+}, 854.4044$; found, 854.4143.

\section{Ammonium salt of benzophenone-biotin (10).}

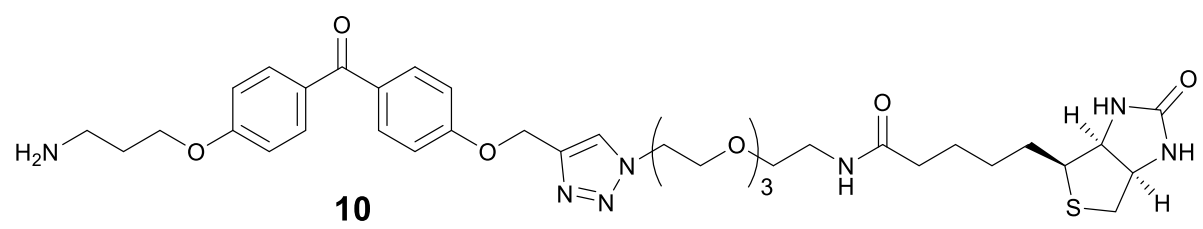

To a $\mathrm{CH}_{2} \mathrm{Cl}_{2}$ solution $(2 \mathrm{~mL})$ of the Boc-protected benzophenone-biotin 9 (20 mg, $\left.0.028 \mathrm{mmol}\right)$ was added TFA $(0.4 \mathrm{~mL})$ at $0{ }^{\circ} \mathrm{C}$. After stirring for $2.5 \mathrm{~h}$ at room temperature, the reaction mixture was concentrated under reduced pressure to afford the crude amine 10 (16 $\mathrm{mg}, 72 \%)$. The crude ammonium salt was used for the next reaction without further purification. ${ }^{1} \mathrm{H}-\mathrm{NMR}(600 \mathrm{MHz}$, $\left.\mathrm{CD}_{3} \mathrm{OD}\right) \delta 8.17(\mathrm{~s}, 1 \mathrm{H}), 7.77(\mathrm{~d}, 4 \mathrm{H}, J=8.4 \mathrm{~Hz}), 7.16(\mathrm{~d}, 2 \mathrm{H}, J=8.4 \mathrm{~Hz}), 7.08(\mathrm{~d}, 2 \mathrm{H}, J=8.4 \mathrm{~Hz})$, $5.28(\mathrm{~s}, 2 \mathrm{H}), 4.62(\mathrm{~m}, 4 \mathrm{H}), 4.23(\mathrm{t}, 2 \mathrm{H}, J=6.0 \mathrm{~Hz}), 3.91(\mathrm{t}, 2 \mathrm{H}, J=5.0 \mathrm{~Hz}), 3.65(\mathrm{~m}, 2 \mathrm{H}), 3.59-3.55$ (m, 8H), $3.48(\mathrm{~m}, 2 \mathrm{H}), 3.18(\mathrm{t}, 2 \mathrm{H}, J=12 \mathrm{~Hz}), 3.12-3.01(\mathrm{~m}, 2 \mathrm{H}), 2.20(\mathrm{~m}, 4 \mathrm{H}), 1.89(\mathrm{~m}, 2 \mathrm{H}), 1.66$ (m, 4H); ${ }^{13} \mathrm{C}-\mathrm{NMR}\left(150 \mathrm{MHz}, \mathrm{CD}_{3} \mathrm{OD}\right) \delta 165.1,174.8,164.7,162.1,161.8,159.2,158.9,132.0$, 132.0, 130.7, 130.4, 125.1 , 114.2, 113.9, 70.0, 70.0, 69.9, 69.8, 69.1 , 68.8, 65.1, 62.0, 61.1, 60.3, 55.5, 53.3, 50.2, 39.6, 38.9, 37.1 , 35.3, 28.3, 28.0, 26.8, 25.4; HRMS (ESI): mass calcd for $\mathrm{C}_{37} \mathrm{H}_{51} \mathrm{~N}_{7} \mathrm{O}_{8} \mathrm{~S}[\mathrm{M}$ $+\mathrm{H}]^{+}, 754.3520$; found, 754.3623 .

tert-butyl 2-(2-me thoxy-5-((5,6,7-trimethoxy-4-oxochroman-3-yl)methyl)phenoxy)acetate (12)<smiles>COC(=O)COc1cc(CC2COc3cc(OC)c(OC)c(OC)c3C2=O)ccc1OC</smiles>

12

To an acetone solution (5 mL) of 3-(3-hydroxy-4-methoxybenzyl)-5,6,7-trimethoxychroman-4-one (11) (102 mg, $0.272 \mathrm{mmol}$ ) were added tert-butyl bromoacetate ( $48 \mu \mathrm{L}, 0.326 \mathrm{mmol})$ and $\mathrm{K}_{2} \mathrm{CO}_{3}(104$ $\mathrm{mg}, 0.816 \mathrm{mmol})$. After refluxing for $3 \mathrm{~h}$, the reaction mixture was diluted with ethyl acetate and the combined organic phase was washed with water and brine, dried over $\mathrm{MgSO}_{4}$ and concentrated under reduced pressure. The residue was purified by flash column chromatography on silica gel (ethyl 
acetate $/ n$-hexane $=1: 2)$ to afford the tert-butyl aryloxyacetate (12) $(105 \mathrm{mg}, 79 \%)$. ${ }^{1} \mathrm{H}-\mathrm{NMR}(600$ $\left.\mathrm{MHz}, \mathrm{CDCl}_{3}\right) \delta 6.78(\mathrm{t}, 2 \mathrm{H}, J=8.4 \mathrm{~Hz}), 6.61(\mathrm{~d}, 1 \mathrm{H}, J=1.8 \mathrm{~Hz}), 6.16(\mathrm{~s}, 1 \mathrm{H}), 4.51(\mathrm{~s}, 2 \mathrm{H}), 4.20(\mathrm{dd}$, $1 \mathrm{H}, J=11.4$ and $4.2 \mathrm{~Hz}), 4.02(\mathrm{dd}, 1 \mathrm{H}, J=11.4$ and $7.8 \mathrm{~Hz}), 3.88(\mathrm{~s}, 3 \mathrm{H}), 3.81(\mathrm{~s}, 3 \mathrm{H}), 3.79(\mathrm{~s}, 3 \mathrm{H})$, $3.74(\mathrm{~s}, 3 \mathrm{H}), 3.11(\mathrm{dd}, 1 \mathrm{H}, J=13.8$ and $4.2 \mathrm{~Hz}), 2.66(\mathrm{~m}, 1 \mathrm{H}), 2.55(\mathrm{dd}, 1 \mathrm{H}, J=13.8$ and $10.8 \mathrm{~Hz})$; ${ }^{13} \mathrm{C}-\mathrm{NMR}\left(150 \mathrm{MHz}, \mathrm{CDCl}_{3}\right) \delta 193.2,169.6,161.7,161.3,156.4,150.2,149.3,139.4,132.7,124.4$, 116.6, 114.1, 110.6, 97.9, 84.1, 70.9, 68.4, 63.5, 63.2, 58.0, 58.0, 50.4, 34.3, 30.0, 30.0.

\section{2-(2-methoxy-5-((5,6,7-trimethoxy-4-oxochroman-3-yl)methyl)phenoxy)acetic acid (13)}<smiles>COc1ccc(CC2COc3cc(OC)c(OC)c(OC)c3C2=O)cc1OCC(=O)O</smiles>

To a $\mathrm{CH}_{2} \mathrm{Cl}_{2}$ solution (2.0 mL) of the tert-butyl acetate (12) (66 mg, $0.135 \mathrm{mmol}$ ) was added TFA $(0.7 \mathrm{~mL})$. After stirring for $2.5 \mathrm{~h}$ at room temperature, the reaction mixture was concentrated under reduced pressure. The residue was purified by flash column chromatography on silica gel $(\mathrm{MeOH} /$ $\left.\mathrm{CH}_{2} \mathrm{Cl}_{2}=1: 10\right)$ to afford the aryloxyacetic acid (13) $(62 \mathrm{mg}, 84 \%) .{ }^{1} \mathrm{H}-\mathrm{NMR}\left(600 \mathrm{MHz}, \mathrm{CDCl}_{3}\right) \delta$ $6.83(\mathrm{~s}, 2 \mathrm{H}), 6.77(\mathrm{~s}, 1 \mathrm{H}), 6.24(\mathrm{~s}, 1 \mathrm{H}), 4.70(\mathrm{~s}, 2 \mathrm{H}), 4.25(\mathrm{dd}, 1 \mathrm{H}, J=11.4$ and $3.6 \mathrm{~Hz}), 4.09(\mathrm{dd}, 1 \mathrm{H}$, $J=11.4$ and $6 \mathrm{~Hz}$ ), $3.89(\mathrm{~s}, 3 \mathrm{H}), 3.86(\mathrm{~s}, 3 \mathrm{H}), 3.83(\mathrm{~s}, 3 \mathrm{H}), 3.78(\mathrm{~s}, 3 \mathrm{H}), 3.05(\mathrm{dd}, 1 \mathrm{H}, J=13.2$ and $4.2 \mathrm{~Hz}), 2.75(\mathrm{~m}, 1 \mathrm{H}), 2.69$ (dd, 1H, $J=13.2$ and $10.8 \mathrm{~Hz}) ;{ }^{13} \mathrm{C}-\mathrm{NMR}\left(150 \mathrm{MHz}, \mathrm{CDCl}_{3}\right) \delta 194.1$, $173.5,160.5,160.4,154.2$, 148.3, 146.8, 137.1, 130.7, 123.8, 116.4, 112.3, 107.9, 96.2, 68.6, 66.8, 61.8, 61.4, 56.2, 55.9, 48.0, 32.7; HRMS (ESI): mass calcd for $\mathrm{C}_{22} \mathrm{H}_{24} \mathrm{O}_{9}[\mathrm{M}+\mathrm{Na}]^{+}$, 455.1420; found, 455.1419.

N-(2-(2-(2-(2-(4-((4-(4-(3-)(2-(2-methoxy-5-((5,6,7-trime thoxy-4-oxochroman-3yl)me thyl)phe noxy)acetamido)propoxy)benzoyl)phenoxy)methyl)-1H-1,2,3-triazol-1yl)ethoxy)ethoxy)ethoxy)ethyl)-5-((3aS,4S,6aR)-2-oxohexahydro-1H-thieno[3,4-d]imidazol-4yl)pentanamide (3)<smiles>COc1ccc(CC2COc3cc(OC)c(OC)c(OC)c3C2=O)cc1OCC(=O)NCCCOc1ccc(C(=O)c2ccc(OCc3cn(CCOC(C)C)nn3)cc2)cc1</smiles>

To a DMF solution (1 mL) of the carboxylic acid (13) (24 mg, $0.044 \mathrm{mmol})$ were added HBTU (20 $\mathrm{mg}, 0.051 \mathrm{mmol})$ and DIPEA $(22 \mu \mathrm{L}, 0.129 \mathrm{mmol})$. After stirring for $30 \mathrm{~min}$, a DMF solution $(0.5$ 
$\mathrm{mL}$ ) of the ammonium salt (9) (38 $\mathrm{mg}, 0.043 \mathrm{mmol})$ was added to the reaction mixture. After stirring for $24 \mathrm{~h}$, the reaction mixture was diluted with ethyl acetate, dried over $\mathrm{MgSO}_{4}$ and concentrated under reduced pressure. The residue was purified by flash column chromatography on silica gel $\left(\mathrm{MeOH} / \mathrm{CH}_{2} \mathrm{Cl}_{2}=1: 10\right)$ to afford the cremastranone-benzophenone-biotin (3) $(16 \mathrm{mg}, 32 \%) .{ }^{1} \mathrm{H}$ NMR (400 MHz, DMSO-d $\left.{ }_{6}\right) \delta 8.21(\mathrm{~s}, 1 \mathrm{H}), 8.01(\mathrm{t}, 1 \mathrm{H}, J=5.6 \mathrm{~Hz}), 7.78(\mathrm{t}, 1 \mathrm{H}, J=5.6 \mathrm{~Hz}), 7.66(\mathrm{dd}$, $4 \mathrm{H}, J=8.8$ and $6.8 \mathrm{~Hz}), 7.16(\mathrm{~d}, 2 \mathrm{H}, J=8.8 \mathrm{~Hz}), 6.99(\mathrm{~d}, 2 \mathrm{H}, J=8.8 \mathrm{~Hz}), 6.89$ (d, 1H, $J=8.4 \mathrm{~Hz})$, $6.80(\mathrm{~s}, 1 \mathrm{H}), 6.77(\mathrm{~d}, 1 \mathrm{H}, J=8.4 \mathrm{~Hz}), 6.38(\mathrm{~s}, 2 \mathrm{H}), 6.32(\mathrm{~s}, 1 \mathrm{H}), 5.22(\mathrm{~s}, 2 \mathrm{H}), 4.53(\mathrm{t}, 2 \mathrm{H}, J=5.2 \mathrm{~Hz})$, 4.43 (s, 2H), 4.26-4.23 (m, 1H), 4.21 (dd, $1 \mathrm{H}, J=11.2$ and $4.4 \mathrm{~Hz}), 4.08-3.99$ (m, 4H), 3.80 (d, 2H, $J$ $=5.2 \mathrm{~Hz}), 3.77(\mathrm{~s}, 3 \mathrm{H}), 3.71(\mathrm{~s}, 3 \mathrm{H}), 3.69(\mathrm{~s}, 3 \mathrm{H}), 3.60(\mathrm{~s}, 3 \mathrm{H}), 3.50-3.47(\mathrm{~m}, 2 \mathrm{H}), 3.44-3.42(\mathrm{~m}, 6 \mathrm{H})$, 3.34-3.31 (m, 4H), $3.14(\mathrm{q}, 2 \mathrm{H}, J=5.6 \mathrm{~Hz}), 3.05-3.01(\mathrm{~m}, 1 \mathrm{H}), 2.99(\mathrm{dd}, 1 \mathrm{H}, J=14.0$ and $4.8 \mathrm{~Hz})$, 2.78-2.74 (m, 2H), $2.54(\mathrm{~d}, 1 \mathrm{H}, J=12.4 \mathrm{~Hz}), 2.00(\mathrm{t}, 2 \mathrm{H}, J=7.2 \mathrm{~Hz}), 1.90(\mathrm{t}, 2 \mathrm{H}, J=6.4 \mathrm{~Hz})$, $1.56(\mathrm{~m}, 1 \mathrm{H}), 1.48(\mathrm{~m}, 3 \mathrm{H}), 1.24(\mathrm{~m}, 2 \mathrm{H}) ;{ }^{13} \mathrm{C}-\mathrm{NMR}\left(100 \mathrm{MHz}, \mathrm{DMSO}-\mathrm{d}_{6}\right) \delta 193.5,190.5,172.5$, 168.3, 163.1, 162.2, 161.7, 159.5, 159.2, 153.9, 148.2, 147.5, 142.4, 137.2, 132.2, 132.1, 131.3, 130.6, $130.2,125.5,122.8,115.9,114.8,114.5,112.6,108.6,96.8,70.1,70.0,69.9,69.5,69.3,69.0,68.9$, 66.1, 61.7, 61.6, 61.4, 61.1, 59.5, 56.5, 55.9, 55.8, 49.8, 47.6, 40.2, 38.8, 35.8, 35.5, 31.8, 29.1, 28.6, 28.4, 25.6; HRMS (ESI): mass calcd for $\mathrm{C}_{59} \mathrm{H}_{73} \mathrm{~N}_{7} \mathrm{O}_{16} \mathrm{~S}[\mathrm{M}+\mathrm{H}]^{+}$, 1168.4835; found, 1168.5145.

\section{2-Methoxy-5-((5',6',7'-trimethoxy-4' -oxochroman-3'-yl)methyl)phenyl (2S)-3-(4-}

(benzyloxy)phenyl)-2-((tert-butoxycarbonyl)amino)propanoate (14).

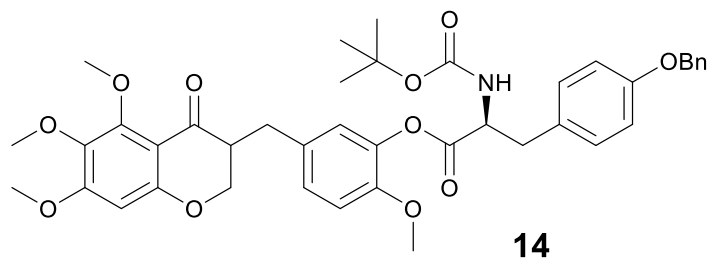

To a $\mathrm{CH}_{2} \mathrm{Cl}_{2}$ solution (2 mL) of $\mathbf{1 1}$ (63 mg, $0.16 \mathrm{mmol}$ ) were added Boc-Tyr(Bzl)-OH (75 mg, 0.19 mmol), EDCI (40 mg, $0.24 \mathrm{mmol})$ and DMAP (4 mg, $0.03 \mathrm{mmol})$. After stirring for $17 \mathrm{~h}$, the reaction mixture was diluted with $\mathrm{CH}_{2} \mathrm{Cl}_{2}$ and washed with water and brine, dried over anhydrous $\mathrm{MgSO}_{4}$ and concentrated under reduced pressure. The residue was purified by flash column chromatography on silica gel (ethyl acetate $: n$-hexane $=1: 2$ ) to afford the acylated 3-benzyl-chroman-4-one (14) (115 mg, 94\%). ${ }^{1} \mathrm{H}-\mathrm{NMR}\left(600 \mathrm{MHz}, \mathrm{CDCl}_{3}\right) \delta 7.39$ (d, 2H, $\left.J=7.2 \mathrm{~Hz}\right), 7.33$ (t, 3H, $\left.J=7.2 \mathrm{~Hz}\right), 7.28$ (t, $1 \mathrm{H}, J=7.2 \mathrm{~Hz}), 7.19(\mathrm{~d}, 2 \mathrm{H}, J=8.4 \mathrm{~Hz}), 7.05(\mathrm{dd}, 1 \mathrm{H}, J=8.4$ and $1.8 \mathrm{~Hz}), 6.92(\mathrm{~m}, 3 \mathrm{H}), 6.22(\mathrm{~d}, 1 \mathrm{H}$, $J=2.4 \mathrm{~Hz}), 5.01(\mathrm{~s}, 2 \mathrm{H}), 4.80(\mathrm{~m}, 1 \mathrm{H}), 4.26(\mathrm{dd}, 1 \mathrm{H}, J=11.4$ and $4.2 \mathrm{~Hz}), 4.06(\mathrm{dd}, 1 \mathrm{H}, J=7.8$ and $3.6 \mathrm{~Hz}$ ), 3.89 (s, 3H), 3.81 (s, 3H), 3.78 (s, 3H), 3.76 (s, 3H), 3.26 (dd, $1 \mathrm{H}, J=14.4$ and $6.0 \mathrm{~Hz}$ ), 3.16 
$(\mathrm{dd}, 1 \mathrm{H}, J=13.8$ and $4.2 \mathrm{~Hz}), 2.72(\mathrm{~m}, 1 \mathrm{H}), 2.62(\mathrm{~m}, 1 \mathrm{H}), 1.40(\mathrm{~s}, 9 \mathrm{H}) ;{ }^{13} \mathrm{C}-\mathrm{NMR}\left(150 \mathrm{MHz}, \mathrm{CDCl}_{3}\right)$ $\delta 191.0,170.1,159.6,159.3,157.9,155.0,154.4,149.6,139.2,137.5,136.9,131.0,130.6,128.5$, 128.2, 127.9, 127.6, 127.4, 123.4, 114.8, 112.6, 108.6, 96.0, 79.9, 70.0, 68.9, 61.6, 61.3, 56.0, 55.8, 54.4, 48.2, 37.3, 31.8, 28.3; HRMS (ESI): mass calcd for $\mathrm{C}_{41} \mathrm{H}_{45} \mathrm{NO}_{11}[\mathrm{M}+\mathrm{H}]^{+}, 727.2993$; found, 727.302 .

\section{2-Methoxy-5-((5,6,7-trimethoxy-4-oxochroman-3-yl)methyl)phe nyl (tert-butoxycarbonyl)-L-} tyrosinate (15)<smiles>COc1ccc(CC2COc3cc(OC)c(OC)c(OC)c3C2=O)cc1OC(=O)C(Cc1ccc(O)cc1)NC(=O)OC(C)(C)C</smiles>

A solution of $14(161 \mathrm{mg}, 0.22 \mathrm{mmol})$ and $10 \% \mathrm{Pd} / \mathrm{C}(23 \mathrm{mg})$ in ethyl acetate $(3 \mathrm{~mL})$ was placed under an atmosphere of hydrogen. After stirring for $48 \mathrm{~h}$, the reaction mixture was diluted with ethyl acetate, filtered through a short pad of silica gel and concentrated under reduced pressure. The residue was purif ied by flash column chromatography on silica gel (ethyl acetate / $n$-hexane $=1: 2$ ) to afford (15) $(112 \mathrm{mg}, 88 \%) .{ }^{1} \mathrm{H}-\mathrm{NMR}\left(600 \mathrm{MHz}, \mathrm{CDCl}_{3}\right) \delta 7.10$ (bs, 2H), 7.01(dd, $1 \mathrm{H}, J=8.4$ and $\left.1.8 \mathrm{~Hz}\right)$, $6.85(\mathrm{~m}, 3 \mathrm{H}), 6.44(\mathrm{bs}, 1 \mathrm{H}), 6.25(\mathrm{~s}, 1 \mathrm{H}), 5.10(\mathrm{~m}, 1 \mathrm{H}), 4.80(\mathrm{~m}, 1 \mathrm{H}), 4.25(\mathrm{~d}, 1 \mathrm{H}, J=9.0 \mathrm{~Hz}), 4.08$ $(\mathrm{m}, 1 \mathrm{H}), 3.92(\mathrm{~s}, 3 \mathrm{H}), 3.86(\mathrm{~s}, 3 \mathrm{H}), 3.79(\mathrm{~s}, 3 \mathrm{H}), 3.75(\mathrm{~s}, 3 \mathrm{H}), 3.17(\mathrm{~m}, 1 \mathrm{H}), 3.09(\mathrm{~m}, 1 \mathrm{H}), 3.03(\mathrm{t}, 1 \mathrm{H}$, $J=10.2 \mathrm{~Hz}), 2.66$ (m, 2H), $1.42(\mathrm{~s}, 9 \mathrm{H}) ;{ }^{13} \mathrm{C}-\mathrm{NMR}\left(150 \mathrm{MHz}, \mathrm{CDCl}_{3}\right) \delta$ 192.0, 170.3, 159.8, 159.7, 155.5, 155.0, 154.4 149.8, 139.2, 137.5, 130.9, 130.8, 127.6, 123.4, 115.8, 112.4, 108.4, 96.1, 80.1, $68.6,61.6,61.3,56.1,55.9,54.7,53.4,48.4,38.0,31.9,28.3$; HRMS (ESI): mass calcd for $\mathrm{C}_{34} \mathrm{H}_{39} \mathrm{NO}_{11}[\mathrm{M}+\mathrm{H}]^{+}, 638.2601$; found, 638.2601.

2-(4-((2S)-2-((tert-Butoxycarbonyl)amino)-3-(2-methoxy-5-((5,6,7-trimethoxy-4-oxochroman-3yl)methyl)phe noxy)-3-oxopropyl)phe noxy)acetic acid (16)<smiles>COc1ccc(CC2COc3cc(OC)c(OC)c(OC)c3C2=O)cc1OC(=O)C(Cc1ccc(OCC(=O)O)cc1)NC(=O)O</smiles> 
To an acetone ( $3 \mathrm{~mL})$ solution of 15 ( $26 \mathrm{mg}, 0.04 \mathrm{mmol})$, benzyl bromoacetate (10 $\mu \mathrm{L}, 0.08 \mathrm{mmol})$ and $\mathrm{K}_{2} \mathrm{CO}_{3}(16 \mathrm{mg}, 0.12 \mathrm{mmol})$ were added. After stirring for $8 \mathrm{~h}$ at room temperature, the reaction mixture was diluted with ethyl acetate and the organic phase was washed with water and brine, dried over anhydrous $\mathrm{MgSO}_{4}$ and concentrated under reduced pressure. The residue was purified by flash column chromatography (ethyl acetate $/ n$-hexanes $=1: 1$ ) to afford the benzyl ester (30 mg, 96\%). And then a solution of benzyl ester (30 mg, $0.038 \mathrm{mmol})$ and $10 \% \mathrm{Pd} / \mathrm{C}(5 \mathrm{mg})$ in ethyl acetate $(3 \mathrm{~mL})$ was placed under an atmosphere of hydrogen. After stirring for $4 \mathrm{~h}$, the reaction mixture was diluted with ethyl acetate, filtered through a short pad of silica gel and concentrated under reduced pressure. The residue was purified by flash column chromatography on silica gel $\left(\mathrm{MeOH} / \mathrm{CH}_{2} \mathrm{Cl}_{2}=1: 10\right)$ to afford 16 (26 mg, 99\%). ${ }^{1} \mathrm{H}-\mathrm{NMR}$ (400 MHz, DMSO-d $\left.{ }_{6}\right) \delta 12.93$ (bs, 1H), 7.42 (d, 1H, J=8 Hz), $7.21(\mathrm{~d}, 2 \mathrm{H}, J=8.4 \mathrm{~Hz}), 7.08(\mathrm{q}, 2 \mathrm{H}, J=8.4 \mathrm{~Hz}), 6.85(\mathrm{~s}, 1 \mathrm{H}), 6.82(\mathrm{~d}, 2 \mathrm{H}, J=8.4 \mathrm{~Hz}), 6.41(\mathrm{~s}, 1 \mathrm{H})$, 4.59 (s, 2H), 4.33-4.28 (m, 1H), $4.27(\mathrm{dd}, 1 \mathrm{H}, J=11.2$ and $4.8 \mathrm{~Hz}), 4.07(\mathrm{t}, 1 \mathrm{H}, J=10 \mathrm{~Hz}), 3.79$ (s, $3 \mathrm{H}), 3.70(\mathrm{~s}, 6 \mathrm{H}), 3.62(\mathrm{~s}, 3 \mathrm{H}), 3.12(\mathrm{dd}, 1 \mathrm{H}, J=13.6$ and $4.4 \mathrm{~Hz}), 3.01(\mathrm{dd}, 1 \mathrm{H}, J=14$ and $5.2 \mathrm{~Hz}$ ), $2.92(\mathrm{dd}, 1 \mathrm{H}, J=14.4$ and $10 \mathrm{~Hz}), 2.85-2.78(\mathrm{~m}, 1 \mathrm{H}), 2.60(\mathrm{dd}, 1 \mathrm{H}, J=13.6$ and $9.2 \mathrm{~Hz}), 1.31$ (s, 9H); ${ }^{13} \mathrm{C}-\mathrm{NMR}\left(100 \mathrm{MHz}, \mathrm{DMSO}-\mathrm{d}_{6}\right) \delta 190.5,170.8,170.7,159.6,159.3,156.9,155.9,154.0,149.7$, 139.4, 137.3, 136.8, 131.5, 130.6, 130.3, 123.5, 114.6, 113.4, 108.6, 96.9, 78.8, 69.4, 64.9, 61.7, 61.7, 61.2, 56.6, 56.3, 47.5, 35.9, 31.3, 28.6; HRMS (ESI): mass calcd for $\mathrm{C}_{36} \mathrm{H}_{41} \mathrm{NO}_{13}[\mathrm{M}+\mathrm{H}]^{+}, 696.2656$; found, 696.2656 .

\section{2-Me thoxy-5-((5,6,7-trimethoxy-4-oxochroman-3-yl)methyl)phe nyl (2S)-2-((tert-}

butoxycarbonyl)amino)-3-(4-(2-oxo-2-((3-(4-(4-((1-(13-0xo-17-((3aS,4S,6aR)-2-oxohexahydro$1 H$-Thieno[3,4-d]imidazol-4-yl)-3,6,9-trioxa-12-azahe ptadecyl)-1H-1,2,3-triazol-4yl)methoxy)benzoyl)phenoxy)propyl)amino)ethoxy)phenyl)propanoate (4a)

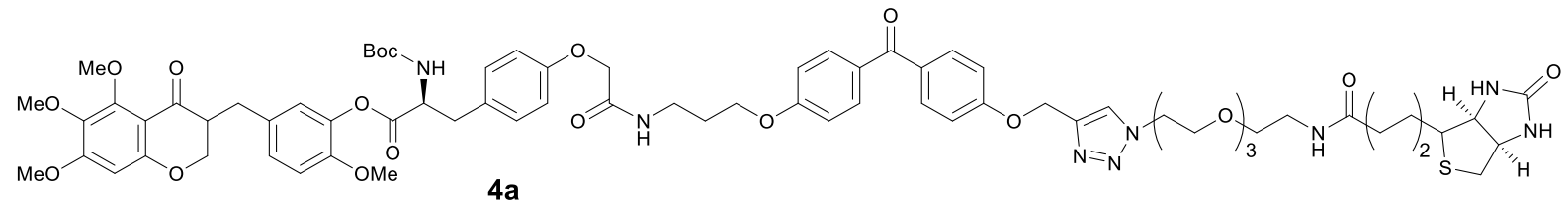

To a solution of 16 (16 mg, $0.023 \mathrm{mmol})$ in DMF (1 mL) at room temperature were added HBTU (10 mg, $0.027 \mathrm{mmol})$ and DIPEA $(12 \mu \mathrm{L}, 0.069 \mathrm{mmol})$. The reaction mixture was stirred at room temperature for $0.5 \mathrm{~h}$ and a solution of benzophenone-biotin amine (10) (20 mg, $0.023 \mathrm{mmol})$ in DMF $(0.5 \mathrm{~mL})$ was added to the mixture. The resulting mixture was stirred at room temperature for $18 \mathrm{~h}$ and quenched at $0{ }^{\circ} \mathrm{C}$ with $\mathrm{H}_{2} \mathrm{O}$. The mixture was extracted three times with ethyl acetate and the 
combined extracts were washed with brine, dried over anhydrous $\mathrm{MgSO}_{4}$ and concentrated under reduced pressure. The residue was purified by flash column chromatography on silica gel $(\mathrm{MeOH} /$ $\left.\mathrm{CH}_{2} \mathrm{Cl}_{2}=1: 30\right)$ to afford the amide (4a) $(22 \mathrm{mg}, 67 \%) .{ }^{1} \mathrm{H}-\mathrm{NMR}\left(400 \mathrm{MHz}, \mathrm{DMSO}-\mathrm{d}_{6}\right) \delta 8.20$ (s, $1 \mathrm{H}), 8.17(\mathrm{t}, 1 \mathrm{H}, J=6 \mathrm{~Hz}), 7.78(\mathrm{t}, 1 \mathrm{H}, J=6 \mathrm{~Hz}), 7.67(\mathrm{~d}, 4 \mathrm{H}, J=8 \mathrm{~Hz}), 7.42(\mathrm{~d}, 1 \mathrm{H}, J=8 \mathrm{~Hz}), 7.23$ $(\mathrm{d}, 2 \mathrm{H}, J=8.4 \mathrm{~Hz}), 7.16(\mathrm{~d}, 2 \mathrm{H}, J=8.8 \mathrm{~Hz}), 7.07-7.00(\mathrm{~m}, 4 \mathrm{H}), 6.88-6.85(\mathrm{~m}, 3 \mathrm{H}), 6.41(\mathrm{~s}, 1 \mathrm{H}), 6.37$ $(\mathrm{s}, 1 \mathrm{H}), 6.31(\mathrm{~s}, 1 \mathrm{H}), 5.21(\mathrm{~s}, 2 \mathrm{H}), 4.52(\mathrm{t}, 2 \mathrm{H}, J=5.6 \mathrm{~Hz}), 4.41(\mathrm{~s}, 2 \mathrm{H}), 4.29(\mathrm{~m}, 1 \mathrm{H}), 4.26-4.22(\mathrm{~m}$, 2H), 4.08-4.01 (m, 4H), $3.79(\mathrm{~m}, 5 \mathrm{H}), 3.69(\mathrm{~s}, 6 \mathrm{H}), 3.61(\mathrm{~s}, 3 \mathrm{H}), 3.49-3.47(\mathrm{~m}, 2 \mathrm{H}), 3.44-3.42(\mathrm{~m}, 5 \mathrm{H})$, $3.34(\mathrm{t}, 2 \mathrm{H}, J=6 \mathrm{~Hz}), 3.27(\mathrm{~d}, 2 \mathrm{H}, J=2 \mathrm{~Hz}), 3.13(\mathrm{~d}, 2 \mathrm{H}, J=6 \mathrm{~Hz}), 3.08(\mathrm{~m}, 1 \mathrm{H}), 3.05(\mathrm{~m}, 1 \mathrm{H})$, 3.00-2.99 (m, 1H), 2.97-2.95 (m, 1H), 2.92-2.86 (m, 1H), $2.80(\mathrm{~m}, 1 \mathrm{H}), 2.78(\mathrm{dd}, 1 \mathrm{H}, J=16.8$ and 4.8 $\mathrm{Hz}), 2.59-2.56(\mathrm{~m}, 1 \mathrm{H}), 2.54(\mathrm{~d}, 1 \mathrm{H}, J=12.4 \mathrm{~Hz}), 2.02(\mathrm{t}, 2 \mathrm{H}, J=6.8 \mathrm{~Hz}), 1.90(\mathrm{t}, 2 \mathrm{H}, J=6.4 \mathrm{~Hz})$, 1.56-1.52 (m, 1H), $1.44(\mathrm{~m}, 3 \mathrm{H}), 1.30(\mathrm{~s}, 9 \mathrm{H}), 1.24(\mathrm{~m}, 2 \mathrm{H}) ;{ }^{13} \mathrm{C}-\mathrm{NMR}\left(100 \mathrm{MHz}, \mathrm{DMSO}-\mathrm{d}_{6}\right) \delta 193.5$, 190.4, 172.5, 170.8, 168.1 163.1, 162.3, 161.7, 159.5, 159.2, 156.8, 155.8, 154.0, 149.6, 142.4, 139.3, 137.2, 132.2, 132.1, 131.4, 130.7, 130.6, 130.5, 130.2, 125.5, 123.4, 114.9, 114.8, 114.6, 113.3, 108.6, $96.8,78.8,70.1,70.0,69.9,69.5,69.3,69.0,67.5,66.1,61.7,61.7,61.7,61.4,61.2,59.6,56.6,56.2$, 55.9, 55.8, 49.8, 47.5, 40.2, 38.8, 35.9, 35.8, 35.5, 31.3, 29.1, 28.6, 28.5, 28.4, 28.2, 25.6; HRMS (ESI): mass calcd for $\mathrm{C}_{73} \mathrm{H}_{90} \mathrm{~N}_{8} \mathrm{O}_{20} \mathrm{~S}[\mathrm{M}+\mathrm{H}]^{+}, 1431.6070$; found, 1431.6067.

tert-Butyl ((2S)-3-(4-(benzyloxy)phenyl)-1-((2-methoxy-5-((5,6,7-trimethoxy-4-oxochroman-3yl)me thyl)phenyl)amino)-1-oxopropan-2-yl)carbamate (18)

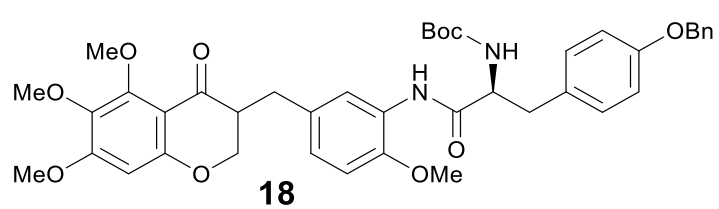

To a $\mathrm{CH}_{2} \mathrm{Cl}_{2}$ solution (6 mL) of $\mathbf{1 7}$ (35 mg, $0.09 \mathrm{mmol}$ ) were added Boc-Tyr(Bn)-OH (35 mg, 0.09 mmol), EDCI (18 mg, $0.1 \mathrm{mmol})$ and DMAP ( $2 \mathrm{mg}, 0.01 \mathrm{mmol})$. After stirring for $17 \mathrm{~h}$, the reaction mixture was diluted with $\mathrm{CH}_{2} \mathrm{Cl}_{2}$ and washed with water and brine, dried over anhydrous $\mathrm{MgSO}_{4}$ and concentrated under reduced pressure. The residue was purified by flash column chromatography on silica gel (ethyl acetate / $n$-hexane $=1: 2)$ to afford $18(50 \mathrm{mg}, 72 \%) .{ }^{1} \mathrm{H}-\mathrm{NMR}\left(600 \mathrm{MHz}, \mathrm{CDCl}_{3}\right) \delta$ 8.22 (s, 1H), 8.10 (bs, 1H), 7.41 (d, 2H, $J=7.8 \mathrm{~Hz}), 7.37$ (t, 2H, $J=7.2 \mathrm{~Hz}), 7.31$ (t, 1H, J=7.2 Hz), $7.16(\mathrm{~d}, 2 \mathrm{H}, J=8.4 \mathrm{~Hz}), 6.90(\mathrm{~d}, 3 \mathrm{H}, J=8.4 \mathrm{~Hz}), 6.75(\mathrm{~d}, 1 \mathrm{H}, J=7.8 \mathrm{~Hz}), 6.24(\mathrm{~s}, 1 \mathrm{H}), 5.01(\mathrm{~s}, 2 \mathrm{H})$, $4.46(\mathrm{bs}, 1 \mathrm{H}), 4.29(\mathrm{dd}, 1 \mathrm{H}, J=10.8$ and $3.6 \mathrm{~Hz}), 4.12(\mathrm{~m}, 1 \mathrm{H}), 3.92(\mathrm{~s}, 3 \mathrm{H}), 3.85(\mathrm{~s}, 3 \mathrm{H}), 3.80$ (s, $3 \mathrm{H}), 3.73(\mathrm{~s}, 3 \mathrm{H}), 3.23(\mathrm{dd}, 1 \mathrm{H}, J=14.4$ and $3.6 \mathrm{~Hz}), 3.10(\mathrm{~m}, 2 \mathrm{H}), 2.81(\mathrm{~m}, 1 \mathrm{H}), 2.63(\mathrm{~m}, 1 \mathrm{H}), 1.42$ (s, 9H); ${ }^{13} \mathrm{C}-\mathrm{NMR}\left(150 \mathrm{MHz}, \mathrm{CDCl}_{3}\right) \delta 191.3,169.4,159.7,159.2,157.8,154.4,146.7,137.4,136.9$, 
131.0, 130.3, 128.5, 127.9, 127.4, 127.0, 124.4, 120.3, 115.0, 110.0, 108.7, 96.0, 70.0, 69.2, 69.2, 61.6, 61.3, 56.0, 55.6, 48.3, 48.3, 37.7, 32.2, 30.9, 29.7, 28.2; HRMS (ESI): mass calcd for $\mathrm{C}_{41} \mathrm{H}_{46} \mathrm{~N}_{2} \mathrm{O}_{10}$ [M $+\mathrm{H}]^{+}, 727.3230$; found, 727.3228.

tert-Butyl (3-(4-hydroxyphenyl)-1-((2-methoxy-5-((5,6,7-trimethoxy-4-oxochroman-3yl)me thyl)phenyl)amino)-1-oxopropan-2-yl)carbamate (19)<smiles>COc1ccc(CC2COc3cc(OC)c(OC)c(OC)c3C2=O)cc1NC(=O)C(Cc1ccccc1)NC(=O)c1ccccc1</smiles>

A solution of $18(50 \mathrm{mg}, 0.068 \mathrm{mmol})$ and $10 \% \mathrm{Pd} / \mathrm{C}(8 \mathrm{mg})$ in ethyl acetate $(3 \mathrm{~mL})$ was placed under an atmosphere of hydrogen. After stirring for $17 \mathrm{~h}$, the reaction mixture was diluted with ethyl acetate, filtered through a short pad of silica gel and concentrated under reduced pressure. The residue was purified by flash column chromatography on silica gel (ethyl acetate / $n$-hexane $=1: 2$ ) to afford 19 (43 mg, 99\%). ${ }^{1} \mathrm{H}-\mathrm{NMR}$ (600 MHz, $\mathrm{CDCl}_{3}$ ) $\delta 8.13$ (s, 1H), 8.03 (bs $\left.1 \mathrm{H}\right), 7.06$ (d, 2H, J = 8.4 Hz), 6.80 (d, 1H, $J=7.8 \mathrm{~Hz}), 6.72$ (m, 3H), 5.14 (bs, 1H), 4.42 (bs, 1H), 4.14 (dd, $1 \mathrm{H}, J=10.4$ and 1.8 $\mathrm{Hz}), 3.95(\mathrm{~d}, 1 \mathrm{H}, J=12.4 \mathrm{~Hz}), 3.88(\mathrm{~d}, 3 \mathrm{H}, J=6.6 \mathrm{~Hz}), 3.80(\mathrm{t}, 6 \mathrm{H}, J=1.2 \mathrm{~Hz}), 3.73(\mathrm{~d}, 3 \mathrm{H}, J=1.8$ $\mathrm{Hz}), 3.15(\mathrm{~m}, 2 \mathrm{H}), 2.50(\mathrm{dd}, 1 \mathrm{H}, J=13.8 \mathrm{~Hz}), 2.40(\mathrm{~m}, 1 \mathrm{H}), 2.25(\mathrm{t}, 1 \mathrm{H}, J=7.8 \mathrm{~Hz}), 1.41(\mathrm{~s}, 9 \mathrm{H})$; ${ }^{13} \mathrm{C}-\mathrm{NMR}\left(150 \mathrm{MHz}, \mathrm{CDCl}_{3}\right) \delta 191.6,169.6,159.8,159.3,155.5,155.3,154.3,146.9,137.3,130.8$, 130.3, 127.7, 126.9, 124.6, 120.5, 115.7, 110.0, 108.6, 96.0, 80.3, 69.2 , 61.6, 61.3, 56.9, 56.1, 55.7, 48.3, 37.8, 32.3, 28.2; HRMS (ESI): mass calcd for $\mathrm{C}_{34} \mathrm{H}_{40} \mathrm{~N}_{2} \mathrm{O}_{10}[\mathrm{M}+\mathrm{H}]^{+}, 637.2761$; found, 637.2758 .

4-(4-((2S)-2-((tert-Butoxycarbonyl)amino)-3-((2-methoxy-5-((5,6,7-trimethoxy-4-oxochroman-3yl)me thyl)phenyl)amino)-3-oxopropyl)phenoxy)butanoic acid (20)

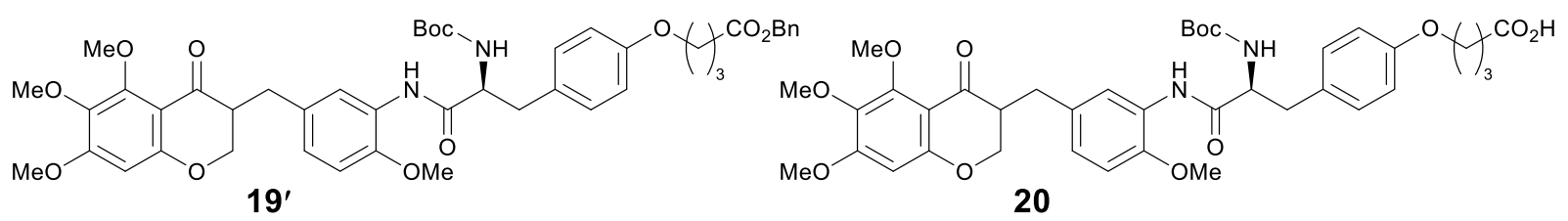

To an acetone $(3 \mathrm{~mL})$ solution of $19(77 \mathrm{mg}, 0.067 \mathrm{mmol})$, benzyl 4-bromobutanoate (28 $\mathrm{mg}, 0.1$ $\mathrm{mmol})$ and $\mathrm{K}_{2} \mathrm{CO}_{3}(30 \mathrm{mg}, 0.2 \mathrm{mmol})$ were added. After refluxing for $24 \mathrm{~h}$, the reaction mixture was 
diluted with ethyl acetate and the organic phase was washed with water and brine, dried over anhydrous $\mathrm{MgSO}_{4}$ and concentrated under reduced pressure. The residue was purified by flash column chromatography (ethyl acetate / $n$-hexanes $=1: 1$ ) to afford the benzyl butanoate 19' (35 mg, 64\%). ${ }^{1} \mathrm{H}-\mathrm{NMR}\left(600 \mathrm{MHz}, \mathrm{CD}_{3} \mathrm{OD}\right) \delta 8.21(\mathrm{~s}, 1 \mathrm{H}), 8.04(\mathrm{bs}, 1 \mathrm{H}), 7.33-7.23(\mathrm{~m}, 5 \mathrm{H}), 7.11(\mathrm{~d}, 2 \mathrm{H}, J=$ $8.4 \mathrm{~Hz}), 6.88(\mathrm{~d}, 1 \mathrm{H}, J=7.8 \mathrm{~Hz}), 6.77(\mathrm{~d}, 2 \mathrm{H}, J=8.4 \mathrm{~Hz}), 6.73(\mathrm{~d}, 1 \mathrm{H}, J=7.8 \mathrm{~Hz}), 6.23(\mathrm{~d}, 1 \mathrm{H}, J=$ $1.8 \mathrm{~Hz}$ ), $5.10(\mathrm{~s}, 2 \mathrm{H}), 4.42(\mathrm{bs}, 1 \mathrm{H}), 4.28(\mathrm{dd}, 1 \mathrm{H}, J=10.8$ and $3.6 \mathrm{~Hz}), 3.94(\mathrm{t}, 2 \mathrm{H}, J=6 \mathrm{~Hz}), 3.90$ (s, $3 \mathrm{H}), 3.85(\mathrm{~s}, 3 \mathrm{H}), 3.79(\mathrm{~s}, 3 \mathrm{H}), 3.71(\mathrm{~s}, 3 \mathrm{H}), 3.22(\mathrm{dd}, 1 \mathrm{H}, J=13.8$ and $3.6 \mathrm{~Hz}), 3.09(\mathrm{bs}, 1 \mathrm{H}), 3.03$ (bs, 1H), 2.78-2.74 (m, 1H), 2.61(t, 1H, $J=13.8 \mathrm{~Hz}), 2.55(\mathrm{t}, 2 \mathrm{H}, J=7.2 \mathrm{~Hz}), 2.10(\mathrm{~m}, 2 \mathrm{H}), 1.41$ (s, 9H); ${ }^{13} \mathrm{C}-\mathrm{NMR}\left(150 \mathrm{MHz}, \mathrm{CD}_{3} \mathrm{OD}\right) \delta$ 191.3, 173.0, 171.1, 169.3, 159.7, 159.2, 154.4, 146.7, 137.4, $135.9,131.0,130.3,128.5,128.2,128.2,124.4,120.3,114.7,110.0,108.7,96.0,69.2,66.6,66.3,61.6$, $61.3,60.4,56.1,55.6,53.4,48.3,48.3,37.7,32.2,31.5,30.8,28.3,24.6,22.6$.

A solution of the benzyl butanoate 19' (30 mg, $0.036 \mathrm{mmol})$ and 10\% Pd/C (10 mg) in ethyl acetate $(3 \mathrm{~mL})$ was placed under an atmosphere of hydrogen. After stirring for $2 \mathrm{~h}$, the reaction mixture was diluted with ethyl acetate, filtered through a short pad of silica gel and concentrated under reduced pressure. The residue was purified by flash column chromatography on silica gel (ethyl acetate / nhexane $=1: 1)$ to afford $20(23 \mathrm{mg}, 86 \%) .{ }^{1} \mathrm{H}-\mathrm{NMR}\left(400 \mathrm{MHz}, \mathrm{DMSO}-\mathrm{d}_{6}\right) \delta 9.04(\mathrm{~s}, 1 \mathrm{H}), 7.89(\mathrm{~s}$, 1H), $7.23(\mathrm{~d}, 1 \mathrm{H}, J=7.6 \mathrm{~Hz}), 7.18(\mathrm{~d}, 2 \mathrm{H}, J=7.6 \mathrm{~Hz}), 6.96(\mathrm{~d}, 1 \mathrm{H}, J=8.4 \mathrm{~Hz}), 6.90(\mathrm{~d}, 1 \mathrm{H}, J=8.4$ Hz), $6.80(\mathrm{~d}, 2 \mathrm{H}, J=7.6 \mathrm{~Hz}), 6.42(\mathrm{~s}, 1 \mathrm{H}), 4.26(\mathrm{~m}, 2 \mathrm{H}), 4.10-4.06(\mathrm{~m}, 1 \mathrm{H}), 3.90(\mathrm{t}, 2 \mathrm{H}, J=6 \mathrm{~Hz})$, $3.80(\mathrm{~s}, 3 \mathrm{H}), 3.78(\mathrm{~s}, 3 \mathrm{H}), 3.70(\mathrm{~s}, 3 \mathrm{H}), 3.62(\mathrm{~s}, 3 \mathrm{H}), 2.98-2.95(\mathrm{~m}, 2 \mathrm{H}), 2.71(\mathrm{~m}, 2 \mathrm{H}), 2.57(\mathrm{~m}, 1 \mathrm{H})$, $2.23(\mathrm{t}, 2 \mathrm{H}, J=6.8 \mathrm{~Hz}), 1.86(\mathrm{t}, 2 \mathrm{H}, J=6.8 \mathrm{~Hz}), 1.30(\mathrm{~s}, 9 \mathrm{H})$. A carboxylic acid proton in the butyric acid linked to tyrosine moiety was not observed; ${ }^{13} \mathrm{C}-\mathrm{NMR}\left(100 \mathrm{MHz}, \mathrm{DMSO}-\mathrm{d}_{6}\right) \delta 190.5,171.0$, 159.6, 159.3, 157.6, 155.9, 154.0, 137.2, 130.7, 130.6, 130.2 , 130.2, 127.3, 121.7, 121.6, 121.6, 114.5, 111.5, 108.6, 96.9, 78.8, 69.3, 67.3, 61.7, 61.2, 57.2, 56.6, 56.3, 48.0, 36.4, 32.1, 31.7, 28.6, 25.3; HRMS (ESI): mass calcd for $\mathrm{C}_{38} \mathrm{H}_{46} \mathrm{~N}_{2} \mathrm{O}_{12}[\mathrm{M}+\mathrm{H}]^{+}, 723.3129$; found, 723.3129.

tert-Butyl ((2S)-1-((2-methoxy-5-((5,6,7-trime thoxy-4-oxochroman-3-yl)methyl)phenyl)amino)-1oxo-3-(4-(4-oxo-4-((3-)(4-(4-)(1-(13-oxo-17-((3aS,4S,6aR)-2-oxohexahydro-1H-thieno[3,4d]imidazol-4-yl)-3,6,9-trioxa-12-azaheptadecyl)-1H-1,2,3-triazol-4yl)me thoxy)be nzoyl)phenoxy)propyl)amino)butoxy)phenyl)propan-2-yl)carbamate. (4b)

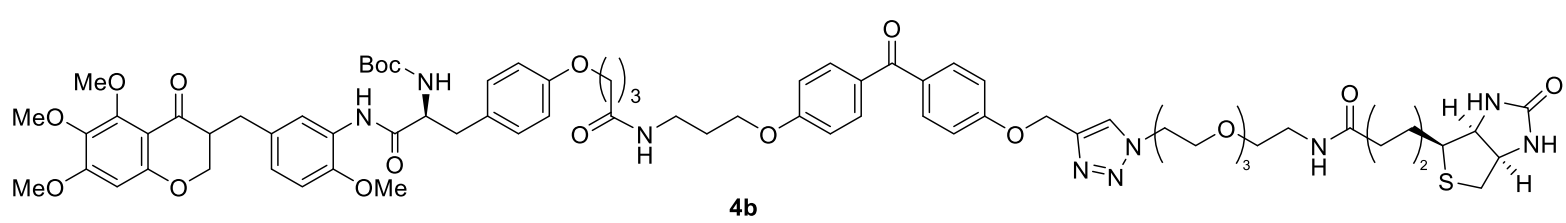


To a solution of $\mathbf{2 0}(20 \mathrm{mg}, 0.028 \mathrm{mmol})$ in DMF $(1 \mathrm{~mL})$ at room temperature were added HBTU (13 $\mathrm{mg}, 0.033 \mathrm{mmol})$ and DIPEA $(15 \mu \mathrm{L}, 0.084 \mathrm{mmol})$. The reaction mixture was stirred at room temperature for $0.5 \mathrm{~h}$ and a solution of benzophenone-biotin amine (9) $(24 \mathrm{mg}, 0.028 \mathrm{mmol})$ in DMF $(0.5 \mathrm{~mL})$ was added to the mixture. The resulting mixture was stirred at room temperature for $18 \mathrm{~h}$ and quenched at $0{ }^{\circ} \mathrm{C}$ with $\mathrm{H}_{2} \mathrm{O}$. The mixture was extracted three times with ethyl acetate and the combined extracts were washed with brine, dried over anhydrous $\mathrm{MgSO}_{4}$ and concentrated under reduced pressure. The residue was purified by flash column chromatography on silica gel $(\mathrm{MeOH} /$ $\mathrm{CH}_{2} \mathrm{Cl}_{2}=1: 30$ ) to afford the amide (4b) $\left(36 \mathrm{mg}, 87 \%\right.$ ). ${ }^{1} \mathrm{H}-\mathrm{NMR}\left(400 \mathrm{MHz}, \mathrm{DMSO}-\mathrm{d}_{6}\right) \delta 9.04$ (s, 1H), $8.20(\mathrm{~s}, 1 \mathrm{H}), 7.94(\mathrm{t}, 1 \mathrm{H}, J=5.2 \mathrm{~Hz}), 7.88(\mathrm{~s}, 1 \mathrm{H}), 7.78(\mathrm{t}, 1 \mathrm{H}, J=5.2 \mathrm{~Hz}), 7.67(\mathrm{~d}, 4 \mathrm{H}, J=7.6$ $\mathrm{Hz}), 7.23(\mathrm{~d}, 1 \mathrm{H}, J=8 \mathrm{~Hz}), 7.18(\mathrm{t}, 4 \mathrm{H}, J=8.8 \mathrm{~Hz}), 7.03(\mathrm{~d}, 2 \mathrm{H}, J=8.8 \mathrm{~Hz}), 6.95(\mathrm{~d}, 1 \mathrm{H}, J=8 \mathrm{~Hz})$, $6.90(\mathrm{~d}, 1 \mathrm{H}, J=8.4 \mathrm{~Hz}), 6.79(\mathrm{~d}, 2 \mathrm{H}, J=8 \mathrm{~Hz}), 6.42(\mathrm{~s}, 1 \mathrm{H}), 6.38(\mathrm{~s}, 1 \mathrm{H}), 6.32(\mathrm{~s}, 1 \mathrm{H}), 5.22(\mathrm{~s}, 2 \mathrm{H})$, $4.52(\mathrm{t}, 2 \mathrm{H}, J=4.8 \mathrm{~Hz}), 4.26(\mathrm{~m}, 3 \mathrm{H}), 4.10-4.02(\mathrm{~m}, 4 \mathrm{H}), 3.88(\mathrm{t}, 2 \mathrm{H} J=6 \mathrm{~Hz}), 3.80(\mathrm{~s}, 4 \mathrm{H}), 3.77$ (s, $3 \mathrm{H}), 3.70(\mathrm{~s}, 3 \mathrm{H}), 3.62(\mathrm{~s}, 3 \mathrm{H}), 3.48(\mathrm{~d}, 2 \mathrm{H}, J=4.4 \mathrm{~Hz}), 3.42(\mathrm{~s}, 5 \mathrm{H}), 3.30(\mathrm{~s}, 8 \mathrm{H}), 3.19(\mathrm{~d}, 2 \mathrm{H}, J=$ $5.6 \mathrm{~Hz}), 3.13(\mathrm{~d}, 2 \mathrm{H}, J=5.6 \mathrm{~Hz}), 3.03(\mathrm{~m}, 1 \mathrm{H}), 2.98(\mathrm{~d}, 2 \mathrm{H}, J=13.2 \mathrm{~Hz}), 2.78-2.70(\mathrm{~m}, 3 \mathrm{H}), 2.57$ (m, 2H), $2.21(\mathrm{t}, 2 \mathrm{H}, J=7.2 \mathrm{~Hz}), 2.02(\mathrm{t}, 2 \mathrm{H}, J=7.2 \mathrm{~Hz}), 1.87(\mathrm{t}, 4 \mathrm{H}, J=6.4 \mathrm{~Hz}), 1.52(\mathrm{~m}, 1 \mathrm{H}), 1.46-$ $1.44(\mathrm{~m}, 3 \mathrm{H}), 1.29$ (s, 9H), 1.22-1.20 (m, 2H); ${ }^{13} \mathrm{C}-\mathrm{NMR}\left(100 \mathrm{MHz}, \mathrm{DMSO}-\mathrm{d}_{6}\right) \delta 193.5,190.5,172.5$, 172.0, 171.0, 163.1, 162.3, 161.7, 159.6, 159.3, 157.5, 155.9, 154.1, 142.4, 137.3, 132.3, 132.2, 130.8, $130.8,130.7,130.6,130.6,130.3,127.3,125.6,124.9,114.9,114.6,114.5,111.4,108.6,96.9,78.8$, $70.1,70.0,69.9,69.6,69.3,69.1,67.2,66.0,61.8,61.8,61.7,61.7,61.4,61.2,59.6,57.2,56.6,56.3$, $56.3,55.8,49.9,48.0,38.8,35.8,35.5,32.2,32.1,31.8,29.2,28.6,28.5,28.4,25.7,25.4$; HRMS (ESI): mass calcd for $\mathrm{C}_{75} \mathrm{H}_{95} \mathrm{~N}_{9} \mathrm{O}_{19} \mathrm{~S}[\mathrm{M}+\mathrm{H}]^{+}, 1458.6543$; found, 1458.6518.

\section{7-Hydroxy-5,6-dimethoxychroman-4-one (22).}

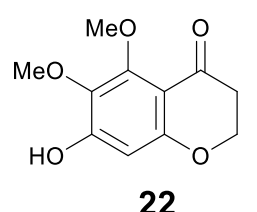

To a solution of 1-(4-(benzyloxy)-6-hydroxy-2,3-dimethoxyphenyl)ethanone 21 (100 mg, 0.33 mmol) in toluene $(2.0 \mathrm{~mL})$ was added $N, N$-dimethylformamide dimethyl acetal $(0.052 \mathrm{~mL}, 0.39$ mmol). After stirring for $18 \mathrm{~h}$ at $80{ }^{\circ} \mathrm{C}$, the mixture was cooled to $0{ }^{\circ} \mathrm{C}$ and $c$ - $\mathrm{HCl}(0.2 \mathrm{~mL})$ was added. After stirring for $1 \mathrm{~h}$ at $50{ }^{\circ} \mathrm{C}$, The reaction mixture was diluted with ethyl acetate and the organic phase was washed with water and brine, and dried over anhydrous $\mathrm{MgSO}_{4}$. The solvent was removed under reduced pressure and purified by flash column chromatograph on silica gel (ethyl 
acetate : $n$-hexane $=1: 2$ ) to afford 7-(benzyloxy)-5,6-dimethoxy-4H-chromen-4-one 21' (101 mg, 97\%). ${ }^{1} \mathrm{H}-\mathrm{NMR}\left(\mathrm{CDCl}_{3}, 600 \mathrm{MHz}\right) \delta 7.63(\mathrm{~d}, 1 \mathrm{H}, J=6.0 \mathrm{~Hz}), 7.46-7.40(\mathrm{~m}, 4 \mathrm{H}), 7.37(\mathrm{t}, 1 \mathrm{H}, J=6.6$ $\mathrm{Hz}), 6.7(\mathrm{~s}, 1 \mathrm{H}), 6.1(\mathrm{~d}, 1 \mathrm{H}, J=6.0 \mathrm{~Hz}), 5.20(\mathrm{~s}, 2 \mathrm{H}), 3.97(\mathrm{~s}, 3 \mathrm{H}), 3.92(\mathrm{~s}, 3 \mathrm{H}) ;{ }^{13} \mathrm{C}-\mathrm{NMR}(150 \mathrm{MHz}$, $\left.\mathrm{CDCl}_{3}\right) \delta 176.2,156.8,154.6,152.9,140.7,135.5,128.8,128.4,127.2,114.2,113.8,97.6,70.9,62.1$, $61.5,30.9$.

An anhydrous $\mathrm{MeOH}$ solution of chromen-4-one 21' (46.8 mg, 0.15mmol) and 10\% Pd/C (15.9mg) was placed under an atmosphere of hydrogen. After stirring for $1 \mathrm{~h}$, the reaction mixture was diluted with EtOAc, filtered through a Celite pad and concentrated under reduced pressure. The residue was purified by flash column chromatography on silica gel (ethyl acetate / $n$-hexanes $=1: 2$ ) to afford the hydrogenated compound 22 (33 mg, 99\%).; ${ }^{1} \mathrm{H}-\mathrm{NMR}\left(\mathrm{CDCl}_{3}, 600 \mathrm{MHz}\right) \delta 6.31(\mathrm{~s}, 2 \mathrm{H}), 4.43(\mathrm{t}, 2 \mathrm{H}, J$ $=6.6 \mathrm{~Hz}), 3.90(\mathrm{~s}, 3 \mathrm{H}), 3.90(\mathrm{~s}, 3 \mathrm{H}), 2.72(\mathrm{~d}, 2 \mathrm{H}, J=6.6 \mathrm{~Hz}) \cdot{ }^{13} \mathrm{C}-\mathrm{NMR}\left(150 \mathrm{MHz}, \mathrm{CDCl}_{3}\right) \delta 189.3$, 160.1 155.5, 153.3, 135.1, 109.6, 98.9, 66.6, 61.5, 61.4, 38.7; HRMS (ESI): mass calcd for $\mathrm{C}_{11} \mathrm{H}_{12} \mathrm{O}_{5}$ $[\mathrm{M}+\mathrm{H}]^{+}, 224.0685$; found, 224.0677.

\section{Benzyl 4-((5,6-dimethoxy-4-oxochroman-7-yl)oxy)butanoate (23)}

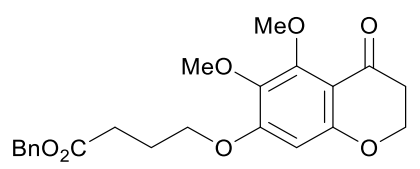

23

To an acetone $(3 \mathrm{~mL})$ solution of 7-hydroxy-5,6-dimethoxychroman-4-one (22) (77 mg, 0.34 mmol), benzyl 4-bromobutanoate ( $88 \mathrm{mg}, 0.34 \mathrm{mmol})$ and $\mathrm{K}_{2} \mathrm{CO}_{3}(142 \mathrm{mg}, 1.0 \mathrm{mmol})$ were added. After refluxing for $24 \mathrm{~h}$, the reaction mixture was diluted with ethyl acetate and the organic phase was washed with water and brine, dried over anhydrous $\mathrm{MgSO}_{4}$ and concentrated under reduced pressure. The residue was purified by flash column chromatography (ethyl acetate / $n$-hexanes $=1: 2$ ) to afford the butanoate (23) $(110 \mathrm{mg}, 80 \%) .{ }^{1} \mathrm{H}-\mathrm{NMR}\left(600 \mathrm{MHz}, \mathrm{CDCl}_{3}\right) \delta 7.30(\mathrm{~s}, 5 \mathrm{H}), 6.18(\mathrm{~s}, 1 \mathrm{H}), 5.09$ (s, 2H), $4.39(\mathrm{t}, 2 \mathrm{H}, J=6.0 \mathrm{~Hz}), 4.01(\mathrm{t}, 2 \mathrm{H}, J=6.0 \mathrm{~Hz}), 3.87(\mathrm{~s}, 3 \mathrm{H}), 3.73(\mathrm{~s}, 3 \mathrm{H}), 2.67(\mathrm{t}, 2 \mathrm{H}, J=6.0$ $\mathrm{Hz}), 2.57(\mathrm{t}, 2 \mathrm{H}, J=7.2 \mathrm{~Hz}), 2.17(\mathrm{~m}, 2 \mathrm{H}) ;{ }^{13} \mathrm{C}-\mathrm{NMR}\left(150 \mathrm{MHz}, \mathrm{CDCl}_{3}\right) \delta 189.1,172.6,159.9,158.5$, $154.4,137.4,135.8,128.5,128.3,128.2,109.6,96.8,67.5,66.8,66.4,61.6,61.2,38.7,30.5,24.2$; HRMS (EI): mass calcd for $\mathrm{C}_{22} \mathrm{H}_{24} \mathrm{O}_{7}[\mathrm{M}+\mathrm{H}]^{+}, 400.1522$; found, 400.1529 . 


\section{Benzyl (E)-4-((3-(3-hydroxy-4-methoxybenzylidene)-5,6-dimethoxy-4-oxochroman-7-}

yl)oxy)butanoate (24)

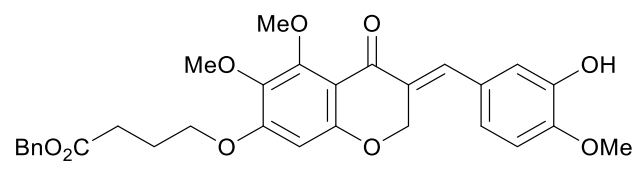

24

To solution of butanoate $(\mathbf{2 3})(56 \mathrm{mg}, 0.139 \mathrm{mmol})$ in benzene $(2 \mathrm{~mL})$ was added isovanillin $(22$ $\mathrm{mg}, 0.139 \mathrm{mmol})$ and $p$-toluenesulfonic acid $(3 \mathrm{mg}, 0.01 \mathrm{mmol})$ at $0{ }^{\circ} \mathrm{C}$. The reaction mixture was refluxed for $20 \mathrm{~h}$. After cooling to room temperature, the reaction mixture was concentrated under reduced pressure. The residue was purified by flash column chromatography on silica gel (ethyl acetate : $n$-hexane $=1: 2)$ to afford $24(40 \mathrm{mg}, 54 \%)$. ${ }^{1} \mathrm{H}-\mathrm{NMR}\left(600 \mathrm{MHz}, \mathrm{CDCl}_{3}\right) \delta 7.71(\mathrm{~s}, 1 \mathrm{H})$, $7.32(\mathrm{~m}, 5 \mathrm{H}), 6.88(\mathrm{~d}, 1 \mathrm{H}, J=8.4 \mathrm{~Hz}), 6.85(\mathrm{~d}, 1 \mathrm{H}, J=1.8 \mathrm{~Hz}), 6.83(\mathrm{dd}, 1 \mathrm{H}, J=8.4$ and $1.8 \mathrm{~Hz})$, $6.19(\mathrm{~s}, 1 \mathrm{H}), 5.21(\mathrm{~s}, 2 \mathrm{H}), 5.11(\mathrm{~s}, 2 \mathrm{H}), 4.04(\mathrm{t}, 2 \mathrm{H}, J=6.0 \mathrm{~Hz}), 3.95(\mathrm{~s}, 3 \mathrm{H}), 3.92(\mathrm{~s}, 3 \mathrm{H}), 3.77(\mathrm{~s}, 3 \mathrm{H})$, 2.59 (t, $2 \mathrm{H}, J=7.2 \mathrm{~Hz}), 2.19(\mathrm{~m}, 2 \mathrm{H}) ;{ }^{13} \mathrm{C}-\mathrm{NMR}\left(150 \mathrm{MHz}, \mathrm{CDCl}_{3}\right) \delta 179.5,172.7,159.3,158.4$, $154.8,147.5,145.5,137.9,136.2,135.8,130.2$, 128.6, 128.3, 128.2, 128.1, 123.2, 115.7, 110.7, 110.5 , 96.9, 67.6, 67.5, 66.4, 61.6, 61.3, 56.0, 30.5, 24.3; HRMS (ESI): mass calcd for $\mathrm{C}_{30} \mathrm{H}_{30} \mathrm{O}_{9}[\mathrm{M}+\mathrm{H}]^{+}$, 535.1968; found, 535.1987.

\section{4-((3-(3-(((tert-B utoxycarbonyl)-L-phenylalanyl)oxy)-4-methoxybenzyl)-5,6-dimethoxy-4-} oxochroman-7-yl)oxy)butanoic acid (25)

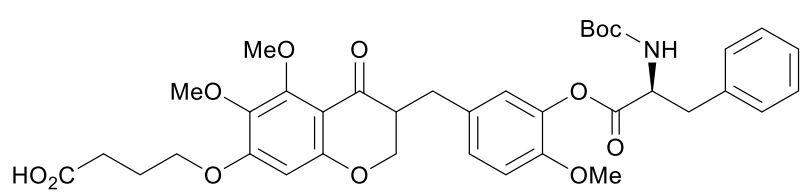

25

To a $\mathrm{CH}_{2} \mathrm{Cl}_{2}$ solution ( $2 \mathrm{~mL}$ ) of benzylidene (24) $(18.8 \mathrm{mg}, 0.035 \mathrm{mmol})$ were added Boc-Phe-OH (10 mg, $0.035 \mathrm{mmol})$, EDCI ( $8 \mathrm{mg}, 0.052 \mathrm{mmol})$ and DMAP (1 mg, $0.007 \mathrm{mmol})$. After stirring for $17 \mathrm{~h}$, the reaction mixture was diluted with $\mathrm{CH}_{2} \mathrm{Cl}_{2}$ and washed with water and brine, dried over anhydrous $\mathrm{MgSO}_{4}$ and concentrated under reduced pressure. The residue was purified by flash column chromatography on silica gel (ethyl acetate / $n$-hexane $=1: 2$ ) to afford the acylated benzylidene 24' (19 mg, 70\%). ${ }^{1} \mathrm{H}-\mathrm{NMR}\left(600 \mathrm{MHz}, \mathrm{CDCl}_{3}\right) \delta 7.70(\mathrm{~s}, 1 \mathrm{H}), 7.33(\mathrm{~m}, 10 \mathrm{H}), 7.16(\mathrm{dd}, 1 \mathrm{H}, J=9.0$ and $2.4 \mathrm{~Hz}), 7.00(\mathrm{~d}, 1 \mathrm{H}, J=9.0 \mathrm{~Hz}), 6.87(\mathrm{~s}, 1 \mathrm{H}), 6.21(\mathrm{~s}, 1 \mathrm{H}), 5.16(\mathrm{~d}, 2 \mathrm{H}, J=1.8 \mathrm{~Hz}), 5.12(\mathrm{~s}, 2 \mathrm{H})$, $4.88(\mathrm{~m}, 1 \mathrm{H}), 4.05$ (t, 2H, J = 6.6 Hz), 3.96 (s, 3H), 3.85 (s, 3H), 3.78 (s, 3H), $3.32(\mathrm{dd}, 1 \mathrm{H}, J=14.4$ 
and $6.0 \mathrm{~Hz}), 3.22(\mathrm{dd}, 1 \mathrm{H}, J=13.8$ and $6.0 \mathrm{~Hz}), 2.60(\mathrm{t}, 2 \mathrm{H}, J=7.2 \mathrm{~Hz}), 2.20(\mathrm{~m}, 2 \mathrm{H}), 1.41(\mathrm{~s}, 9 \mathrm{H})$; ${ }^{13} \mathrm{C}-\mathrm{NMR}\left(150 \mathrm{MHz}, \mathrm{CDCl}_{3}\right) \delta 179.3,172.7,171.1,170.0,159.3,158.5,154.9,151.8,139.2,137.9$, $135.8,134.9,130.7,129.5,128.6,128.3,128.2,127.5,127.1,124.1,112.4,110.6,96.9,67.6,67.4$, 66.4, 61.6, 61.3 , 55.9, 54.3, 38.2, 31.5, 30.5, 29.9, 29.7, 28.3, 28.1, 24.3; HRMS (ESI): mass calcd for $\mathrm{C}_{44} \mathrm{H}_{47} \mathrm{NO}_{12}[\mathrm{M}+\mathrm{H}]^{+}, 782.3175$; found, 782.3164 .

A solution of the acylated benzylidene 24' (19 mg, $0.024 \mathrm{mmol})$ and 10\% Pd/C (4 mg) in ethyl acetate $(2 \mathrm{~mL})$ was placed under an atmosphere of hydrogen. After stirring for $24 \mathrm{~h}$, the reaction mixture was diluted with ethyl acetate, filtered through a short pad of silica gel and concentrated under reduced pressure. The residue was purified by flash column chromatography on silica gel (ethyl acetate $/ n$-hexane $=1: 2)$ to afford the butanoic acid (25) $(16 \mathrm{mg}, 99 \%) .{ }^{1} \mathrm{H}-\mathrm{NMR}\left(600 \mathrm{MHz}, \mathrm{CDCl}_{3}\right)$ $\delta 7.32(\mathrm{~m}, 5 \mathrm{H}), 7.05(\mathrm{~d}, 1 \mathrm{H}, J=7.8 \mathrm{~Hz}), 6.89(\mathrm{~d}, 1 \mathrm{H}, J=8.4 \mathrm{~Hz}), 6.80(\mathrm{~s}, 1 \mathrm{H}), 6.24(\mathrm{~s}, 1 \mathrm{H}), 4.85(\mathrm{~m}$, $1 \mathrm{H}), 4.24(\mathrm{dd}, 1 \mathrm{H}, J=11.4$ and $3.6 \mathrm{~Hz}), 4.07(\mathrm{~m}, 3 \mathrm{H}), 3.90(\mathrm{~s}, 3 \mathrm{H}), 3.78(\mathrm{~s}, 3 \mathrm{H}), 3.77$ (s, 3H), 3.32 (dd, $1 \mathrm{H}, J=7.8 \mathrm{~Hz}), 3.20(\mathrm{~m}, 2 \mathrm{H}), 2.70(\mathrm{~m}, 1 \mathrm{H}), 2.60(\mathrm{t}, 1 \mathrm{H}, J=12 \mathrm{~Hz}), 2.43(\mathrm{t}, 2 \mathrm{H}, J=7.2 \mathrm{~Hz}), 2.15(\mathrm{~m}$, $2 \mathrm{H})$. A carboxylic acid proton in the C7-butyric acid moiety was not observed; ${ }^{13} \mathrm{C}-\mathrm{NMR}(150 \mathrm{MHz}$, $\left.\mathrm{CDCl}_{3}\right) \delta 191.0,177.4,170.1,159.6,159.0,155.0,154.4,149.6,139.2,137.6,136.0,131.1,129.6$, $128.5,127.6,127.0,123.3,112.6,108.4,96.7,79.9,68.9,68.5,65.0,61.6,61.2,55.8,54.3,52.0,48.2$, 40.8, 38.2, 32.3, 31.9, 29.7, 29.6, 28.3, 25.1; HRMS (ESI): mass calcd for $\mathrm{C}_{37} \mathrm{H}_{43} \mathrm{NO}_{12}[\mathrm{M}+\mathrm{H}]^{+}$, 694.2864; found, 694.2852.

5-((5,6-Dimethoxy-4-0xo-7-(4-0xo-4-((3-(4-(4-((1-(13-0xo-17-((3aS,4S,6aR)-2-oxohexahydro-1Hthie no[3,4-d]imidazol-4-yl)-3,6,9-trioxa-12-azahe ptade cyl)-1H-1,2,3-triazol-4-

yl)me thoxy)benzoyl)phenoxy)propyl)amino)butoxy)chroman-3-yl)methyl)-2-me thoxyphenyl (tert-butoxycarbonyl)-L-phenylalaninate (5)

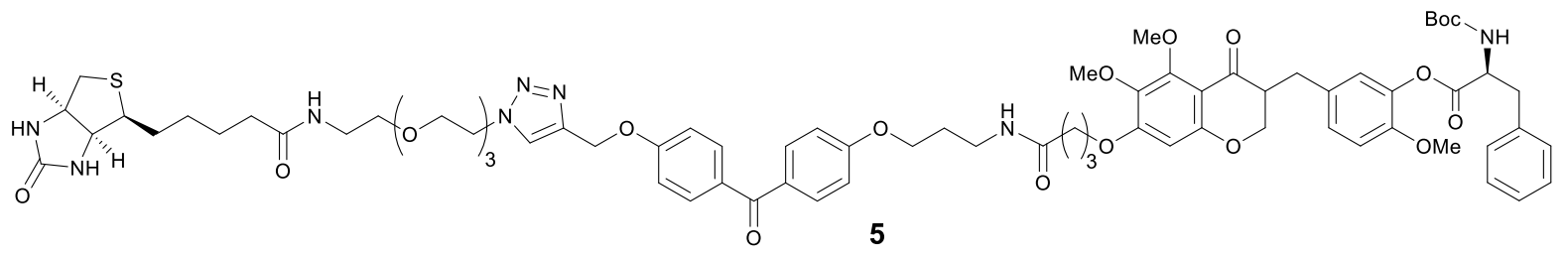

To a solution of 25 (12 mg, $0.016 \mathrm{mmol})$ in DMF (1 mL) at room temperature were added HBTU (9 $\mathrm{mg}, 0.019 \mathrm{mmol})$ and DIPEA $(2 \mu \mathrm{L}, 0.048 \mathrm{mmol})$. The reaction mixture was stirred at room temperature for $0.5 \mathrm{~h}$ and a solution of benzophenone-biotin amine (9) $(14 \mathrm{mg}, 0.016 \mathrm{mmol})$ in DMF $(0.5 \mathrm{~mL})$ was added to the mixture. The resulting mixture was stirred at room temperature for $18 \mathrm{~h}$ and quenched at $0{ }^{\circ} \mathrm{C}$ with $\mathrm{H}_{2} \mathrm{O}$. The mixture was extracted three times with ethyl acetate and the 
combined extracts were washed with brine, dried over anhydrous $\mathrm{MgSO}_{4}$ and concentrated under reduced pressure. The residue was purified by flash column chromatography on silica gel $(\mathrm{MeOH} /$ $\left.\mathrm{CH}_{2} \mathrm{Cl}_{2}=1: 30\right)$ to afford the amide (5) $(8.2 \mathrm{mg}, 36 \%)$. ${ }^{1} \mathrm{H}-\mathrm{NMR}\left(400 \mathrm{MHz}, \mathrm{CDCl}_{3}\right) \delta 7.86(\mathrm{~s}, 1 \mathrm{H})$, $7.75(\mathrm{dd}, 4 \mathrm{H}, J=8.8$ and $2.8 \mathrm{~Hz}), 7.31-7.25(\mathrm{~m}, 4 \mathrm{H}), 7.05(\mathrm{~d}, 3 \mathrm{H}, J=8.8 \mathrm{~Hz}), 6.91(\mathrm{t}, 3 \mathrm{H}, J=8.4$ $\mathrm{Hz}), 6.80(\mathrm{~s}, 1 \mathrm{H}), 6.53(\mathrm{~s}, 1 \mathrm{H}), 6.22(\mathrm{~s}, 1 \mathrm{H}), 6.18(\mathrm{~s}, 1 \mathrm{H}), 5.91(\mathrm{~s}, 1 \mathrm{H}), 5.26(\mathrm{~s}, 2 \mathrm{H}), 5.04(\mathrm{~d}, 1 \mathrm{H}, J=8$ $\mathrm{Hz}), 4.84(\mathrm{~d}, 1 \mathrm{H}, J=6.8 \mathrm{~Hz}), 4.56(\mathrm{t}, 2 \mathrm{H}, J=4.8 \mathrm{~Hz}), 4.45(\mathrm{~m}, 1 \mathrm{H}), 4.25(\mathrm{~m}, 2 \mathrm{H}), 4.08(\mathrm{~m}, 4 \mathrm{H}), 3.88$ (m, 5H), 3.77 (s, 3H), $3.77(\mathrm{~s}, 3 \mathrm{H}), 3.64-3.53(\mathrm{~m}, 10 \mathrm{H}), 3.48-3.44(\mathrm{~m}, 4 \mathrm{H}), 3.39(\mathrm{t}, 2 \mathrm{H}, J=5.2 \mathrm{~Hz})$, $3.33(\mathrm{dd}, 1 \mathrm{H}, J=14.4$ and $6 \mathrm{~Hz}), 3.21-3.09(\mathrm{~m}, 4 \mathrm{H}), 2.86(\mathrm{~m}, 1 \mathrm{H}), 2.72(\mathrm{~m}, 2 \mathrm{H}), 2.61(\mathrm{~m}, 1 \mathrm{H}), 2.42$ $(\mathrm{t}, 2 \mathrm{H}, J=7.6 \mathrm{~Hz}), 2.18-2.12(\mathrm{~m}, 4 \mathrm{H}), 2.03-1.98(\mathrm{~m}, 2 \mathrm{H}), 1.63-1.56(\mathrm{~m}, 4 \mathrm{H}), 1.46-1.39(\mathrm{~m}, 11 \mathrm{H}) ;{ }^{13} \mathrm{C}-$ NMR $\left(100 \mathrm{MHz}, \mathrm{CDCl}_{3}\right) \delta 194.3,191.0,173.5,172.3,171.1,170.1,164.0,162.0,161.4,159.7$, $158.5,154.4,149.6,143.1,139.2,137.4,136.0,132.2$, 132.2 , 131.0, 131.0, 130.5, 129.6, 128.5, 127.6, $127.0,124.4,123.3,114.3,113.9,112.6,108.6,96.9,79.9,70.4,70.3,70.2,70.0,69.9,69.8,69.3$, 68.9, 68.0, 66.1, 61.9, 61.8, 61.7, 61.3, 60.4, 60.2, 55.8, 55.5, 54.3, 50.6, 50.3, 48.1, 42.5, 40.4, 39.1, 38.2, 36.9, 35.7, 32.6, 31.7, 29.0, 28.3, 28.1, 25.5, 24.8; HRMS (ESI): mass calcd for $\mathrm{C}_{74} \mathrm{H}_{92} \mathrm{~N}_{8} \mathrm{O}_{19} \mathrm{SNa}[\mathrm{M}+\mathrm{Na}]^{+}, 1451.6097$; found, 1451.6054 . 


\section{LC-MS analysis of four photoaffinity probes}

LC-MS data were obtained using a Waters system (Waters 2489 UV/Visible Detector, Waters 3100 Mass, Waters 515 HPLC pump, Waters 2545 Binary Gradient Module, Waters Reagent Manager, and Waters 2767 Sample Manager) by using SunFire C18 column $(4.6 \times 50 \mathrm{~mm}, 5 \mu \mathrm{m}$ particle size), isocratic elution at $40 \%$ acetonitrile in water for $60 \mathrm{~min}$; flow rate: $1.4 \mathrm{~mL} / \mathrm{min}$

\begin{tabular}{|l|l|}
\hline LC conditions & MS conditions \\
\hline System : Waters Allance HPLC system & System: Waters 3100 mass detector \\
Flow rate: $1.4 \mathrm{~mL} / \mathrm{min}$ & Mode: Positive electrospray (ES+) \\
Mobile phase A: $\mathrm{H}_{2} \mathrm{O}$ & Capillary: $3.5 \mathrm{kV}$ \\
Mobile phase B: Acetonitrile & Extractor: $1 \mathrm{~V}$ \\
Column: Sunfire $\mathrm{C} 185.0 \mu \mathrm{V} 4.6 \mathrm{x} 50 \mathrm{~mm}$ Column & RF Lens: $0.1 \mathrm{~V}$ \\
Sample diluent: $\mathrm{DMSO}$ & Source temp.: $120{ }^{\circ} \mathrm{C}$ \\
Injection volume: $10 \mu \mathrm{L}$ & Desolvation temp.: $350{ }^{\circ} \mathrm{C}$ \\
PDA scan range: 200 to $600 \mathrm{~nm}$ & Desolvation Gas: $650 \mathrm{~L} / \mathrm{h}$ \\
Data management: MassLynx Software & \\
Isocratic: $40 \%$ Acetonitrile in $\mathrm{H}_{2} \mathrm{O}$ &
\end{tabular}




\section{1) Compound 3}
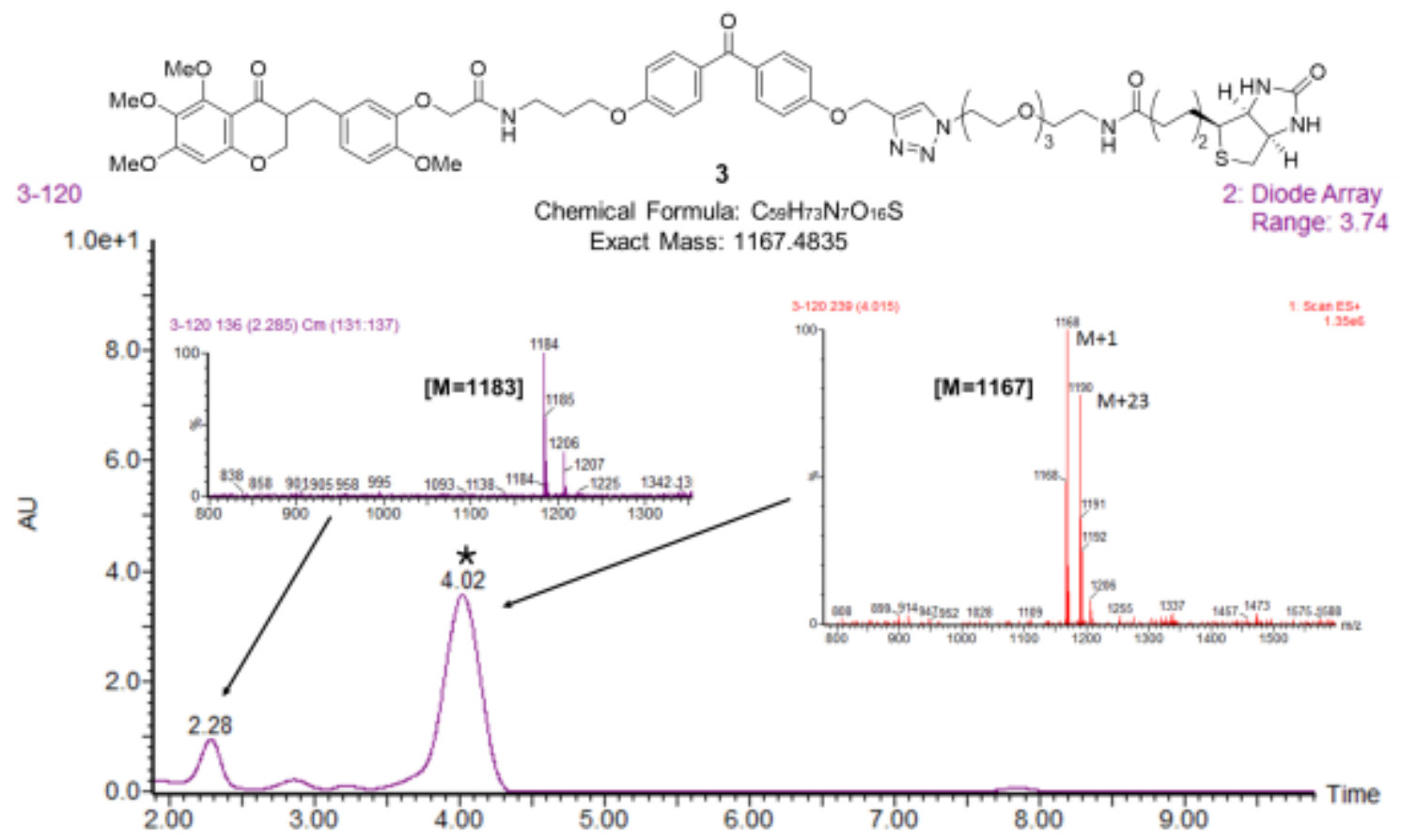

\section{2) Compound 4a}

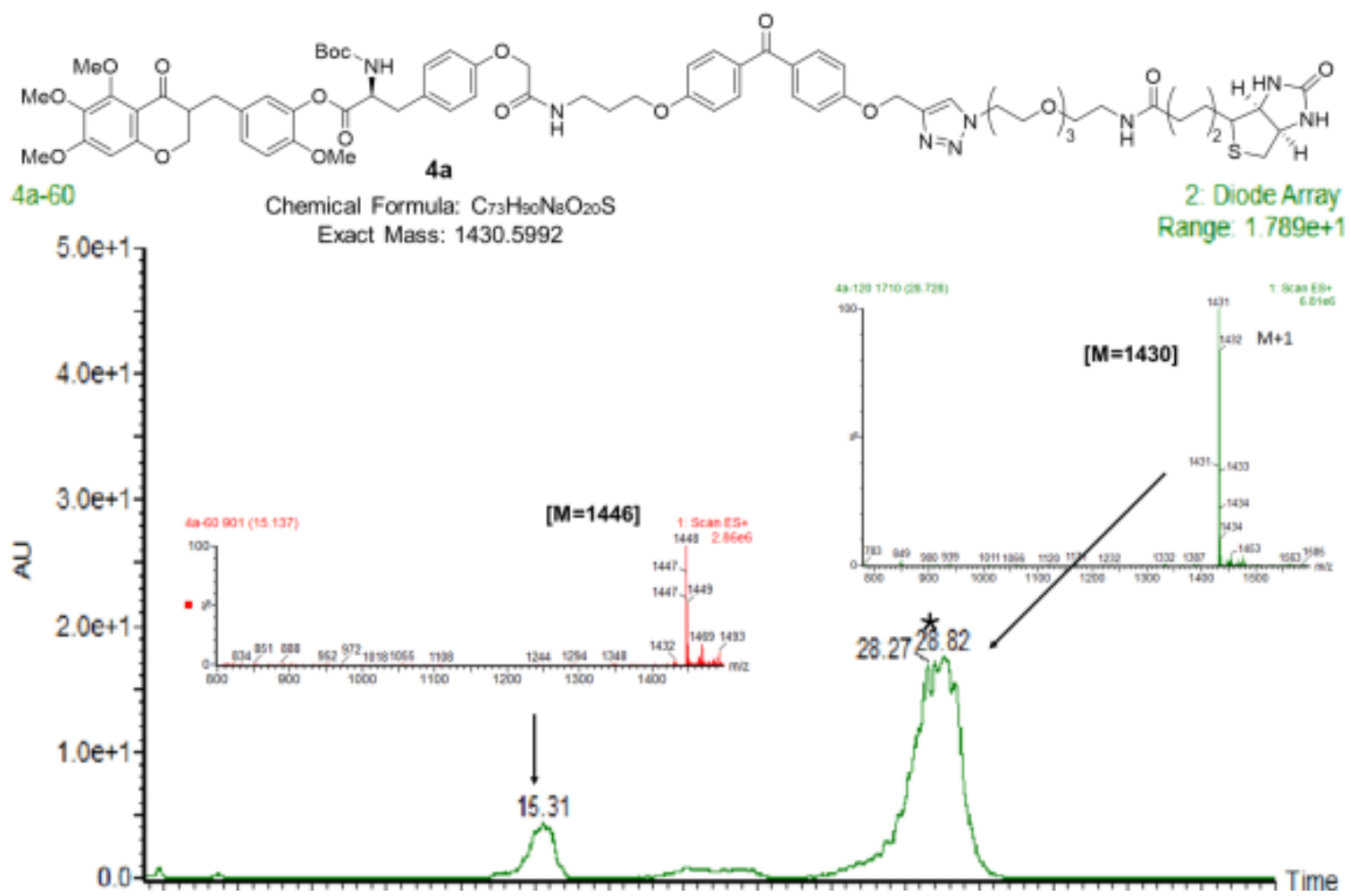




\section{3) Compound 4b}

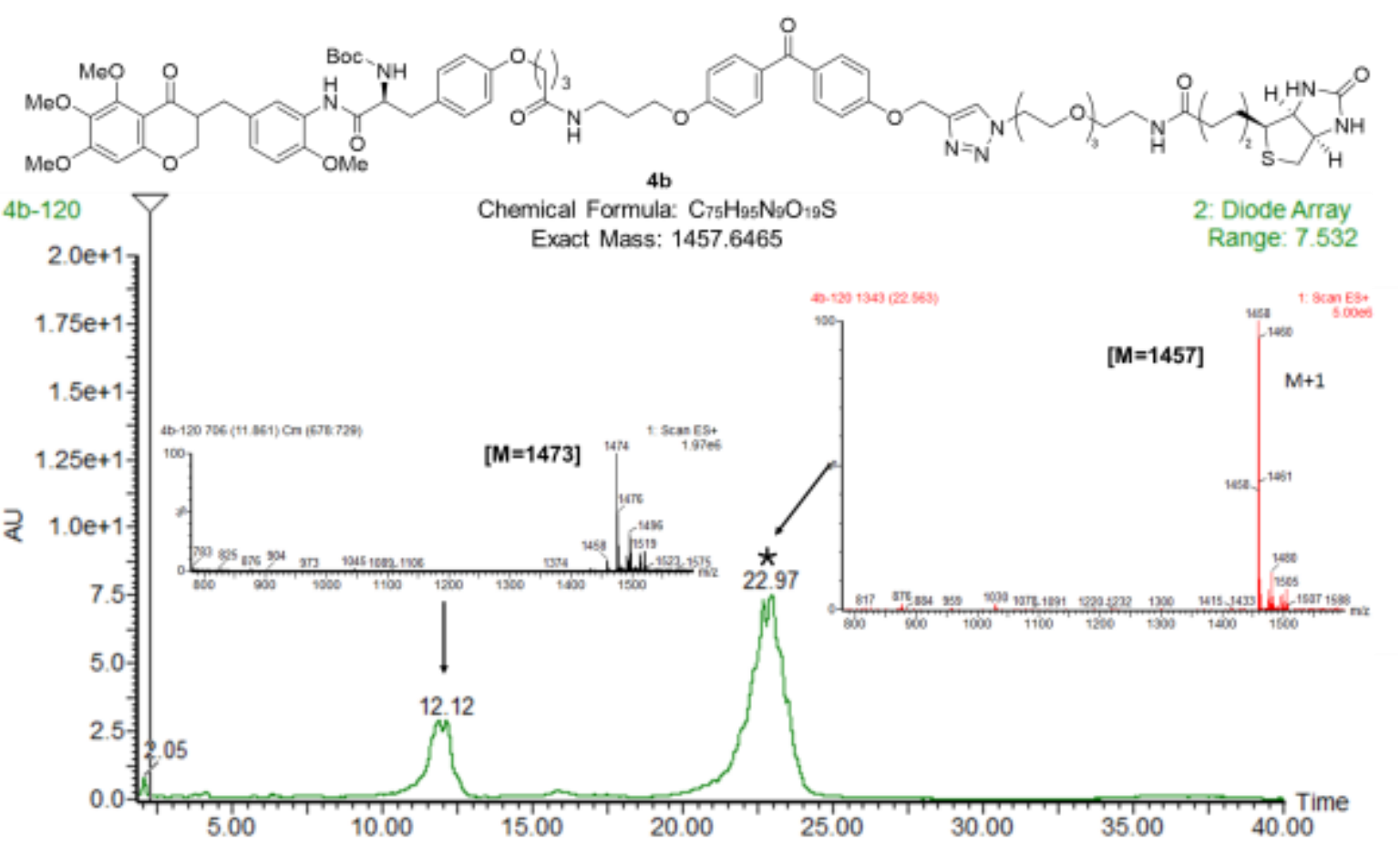

\section{4) Compound 5}

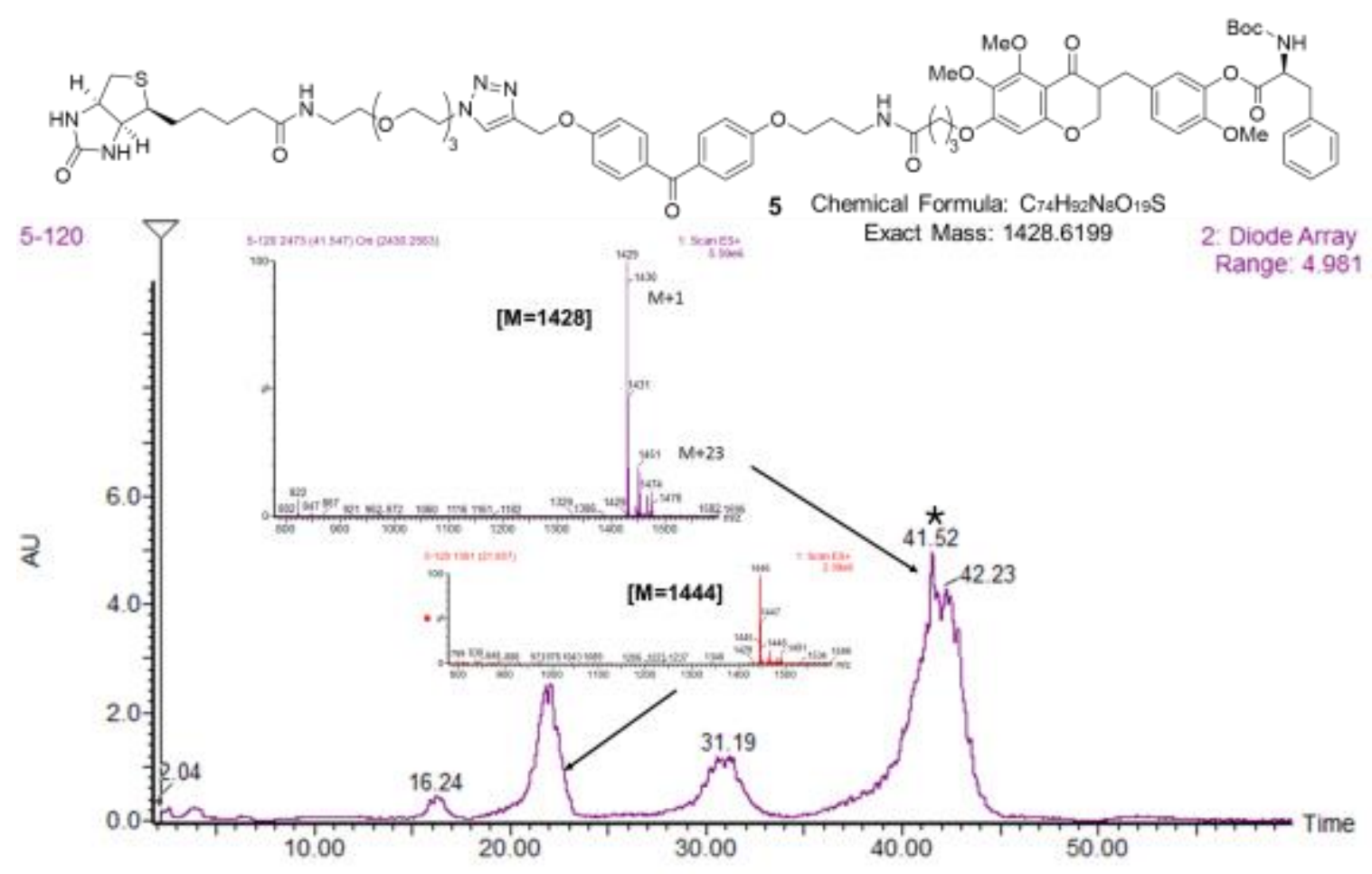




\section{Biological Methods}

\section{Materials}

EBM-2 and IMDM growth media were purchased from Lonza (Walkersville, MD, USA). RPMI and Ham's/F-10 media were purchased from Thermo Scientific (Waltham, MA, USA). HRECs and Attachment Factor were purchased from Cell Systems (Kirkland, WA, USA). Clonetics® HUVECs were purchased from Lonza. All endothelial cells were used between passages 5 and 8. Endothelial Growth Medium (EGM-2) was prepared by mixing the contents of an EGM-2 "Bullet Kit" (Cat. no. CC-4176) with Endothelial Basal Medium (EBM) (Lonza). The EGM-2 "Bullet Kit" contains hydrocortisone, human fibroblast growth factor, vascular endothelial growth factor, R3-insulin like growth factor, ascorbic acid, human epidermal growth factor, gentamycin and heparin along with $2 \%$ fetal bovine serum (FBS). 92-1 cells ${ }^{1}$ (a kind gift from Dr. Martine Jager) were grown in RPMI medium containing 10\% FBS and 1\% penicillin-streptomycin (pen-strep). Y79 cells ${ }^{2}$ (a kind gift from Dr. Brenda Gallie) were grown in RB medium (IMDM + 10\% FBS $+55 \mu \mathrm{M} \beta$-mercaptoethanol + 10 $\mu \mathrm{g} / \mathrm{mL}$ insulin $+1 \%$ pen-strep). ARPE19 cells (ATCC, Manassas, VA, USA) were grown in Ham's/F-10 + 10\% FBS + 1\% pen-strep. Identity of 92-1 and Y79 cell lines was confirmed by short tandem repeat profiling.

\section{Cell Proliferation Assay}

The proliferation of cells was monitored by an alamarBlue based fluorescence assay. Five cell types were used: HRECs, HUVECs, 92-1, Y79 and ARPE19. Briefly, 2,500 cells in 100 $\mu \mathrm{L}$ growth medium were incubated in 96-well clear bottom black plates for 24 hours followed by 48 hours' incubation with different concentrations of each test compound (range: $0.5 \mathrm{nM}$ to $500 \mu \mathrm{M})$. At the end of the incubation, $11.1 \mu \mathrm{L}$ of alamarBlue reagent was added and 4 hours after, fluorescence readings were taken with excitation and emission wavelengths of $560 \mathrm{~nm}$ and $590 \mathrm{~nm}$ respectively. Data were analyzed and dose response curves generated using GraphPad Prism software (v. 6.0). Only those compounds that reduced cell number by $50 \%$ or more at the highest concentration tested (relative to DMSO control) were reported as having a $\mathrm{GI}_{50}<100 \mu \mathrm{M}$.

1. I. De Waard-Siebinga, D. J. Blom, M. Griffioen, P. I. Schrier, E. Hoogendoorn, G. Beverstock, E. H. Danen and M. J. Jager, Int. J. Cancer, 1995, 62, 155-161.

2. T. W. Reid, D. M. Albert, A. S. Rabson, P. Russell, J. Craft, E. W. Chu, T. S. Tralka and J. L. Wilcox, J. Natl. Cancer Inst., 1974, 53, 347-360. 


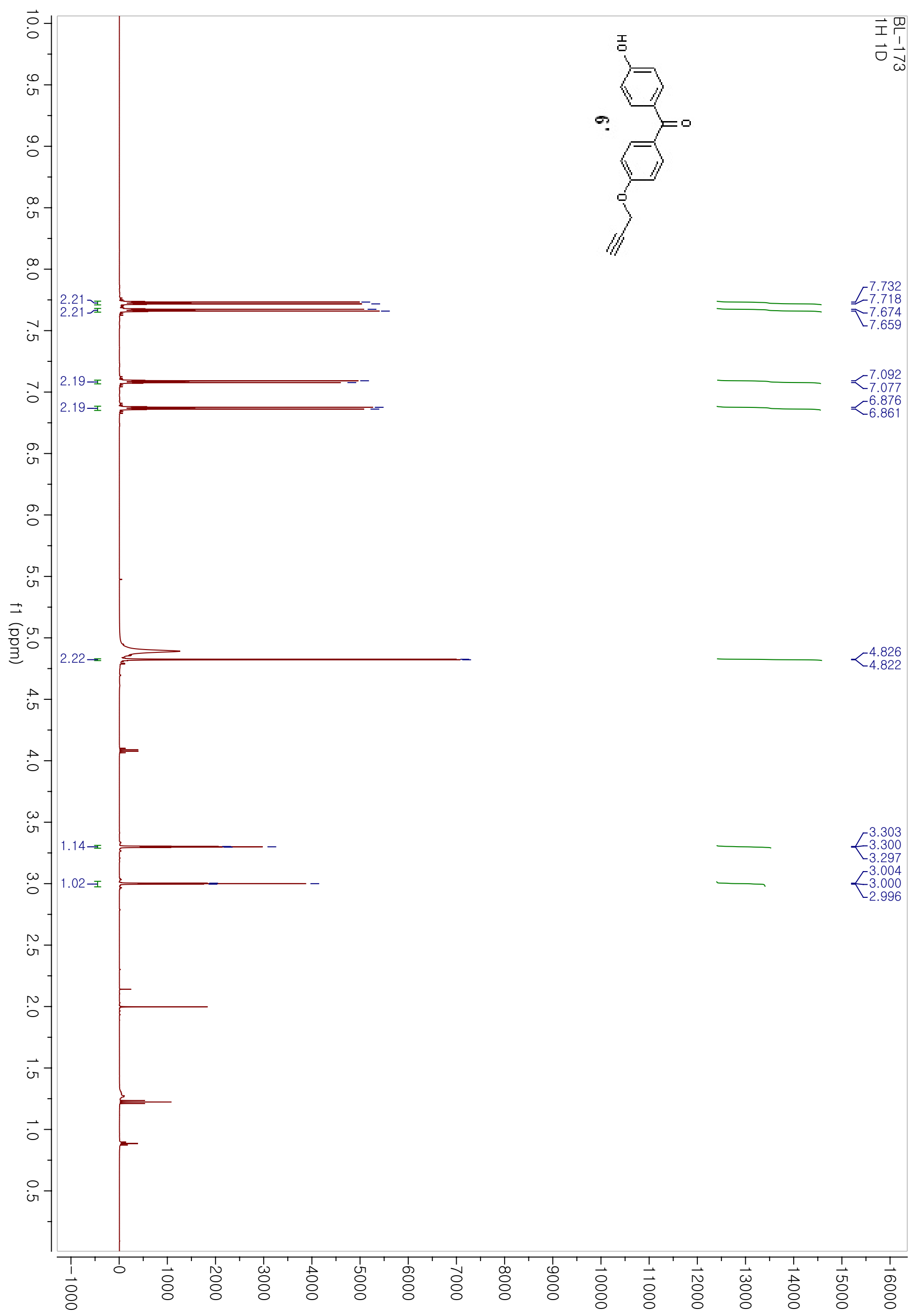




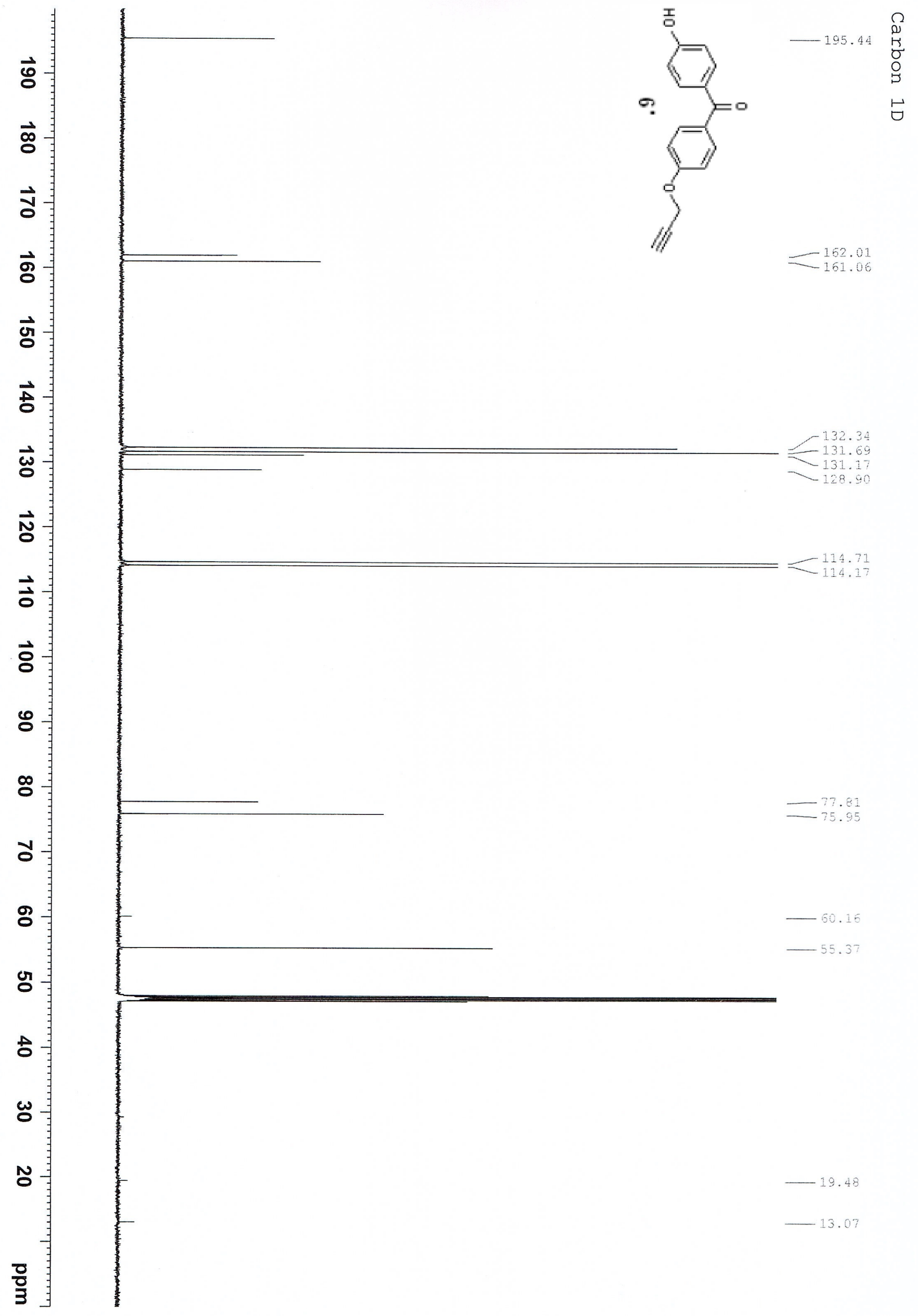




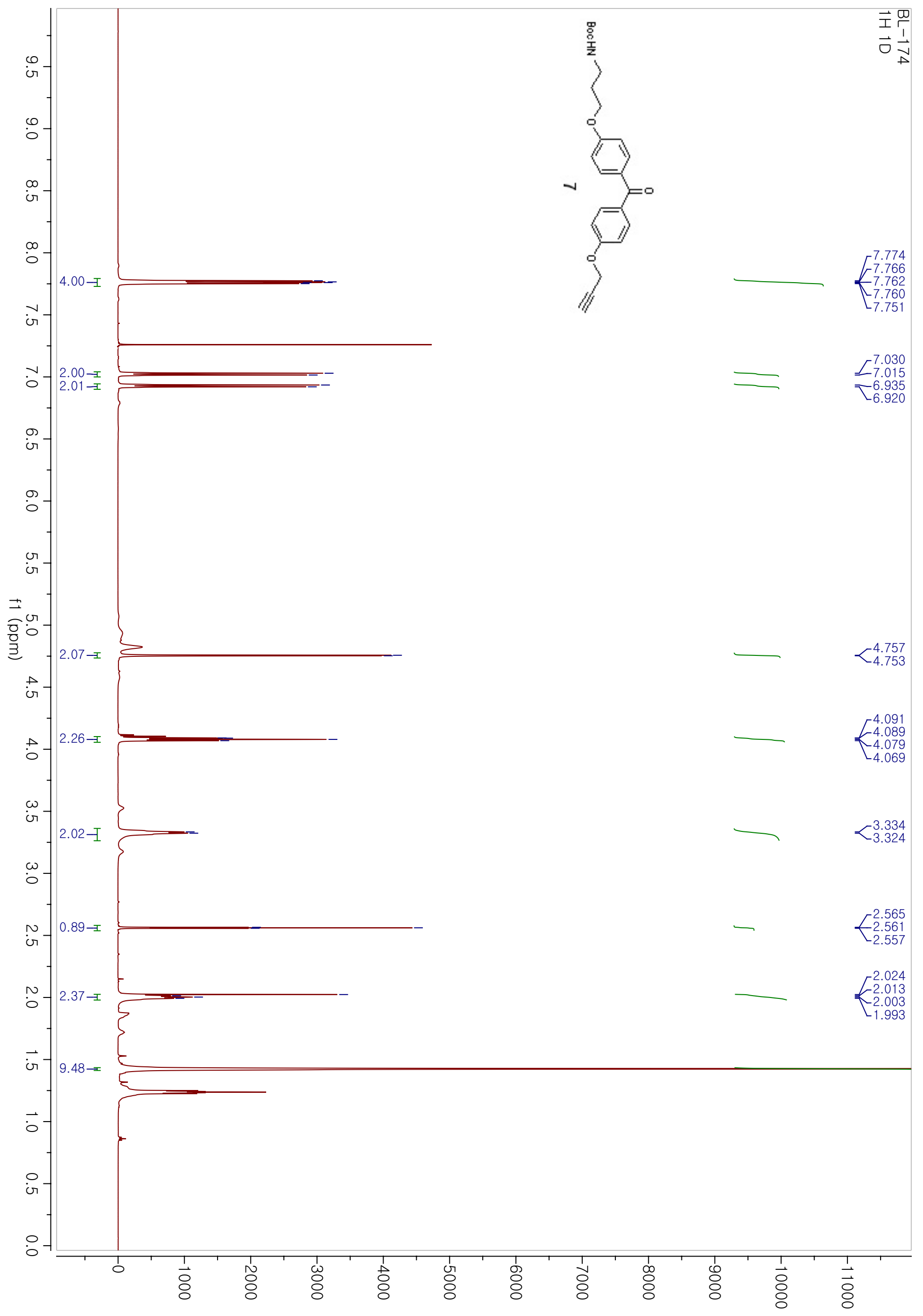




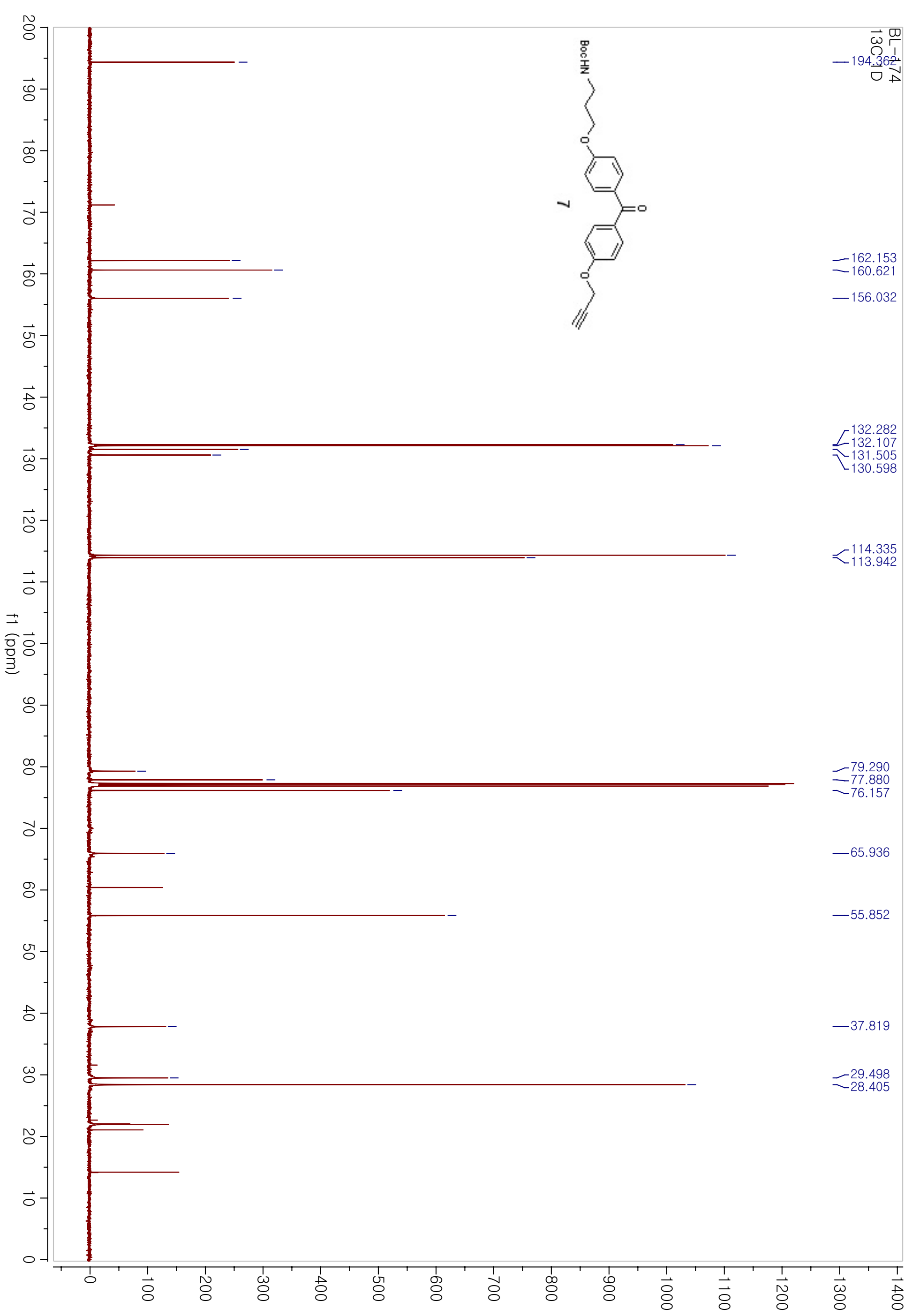




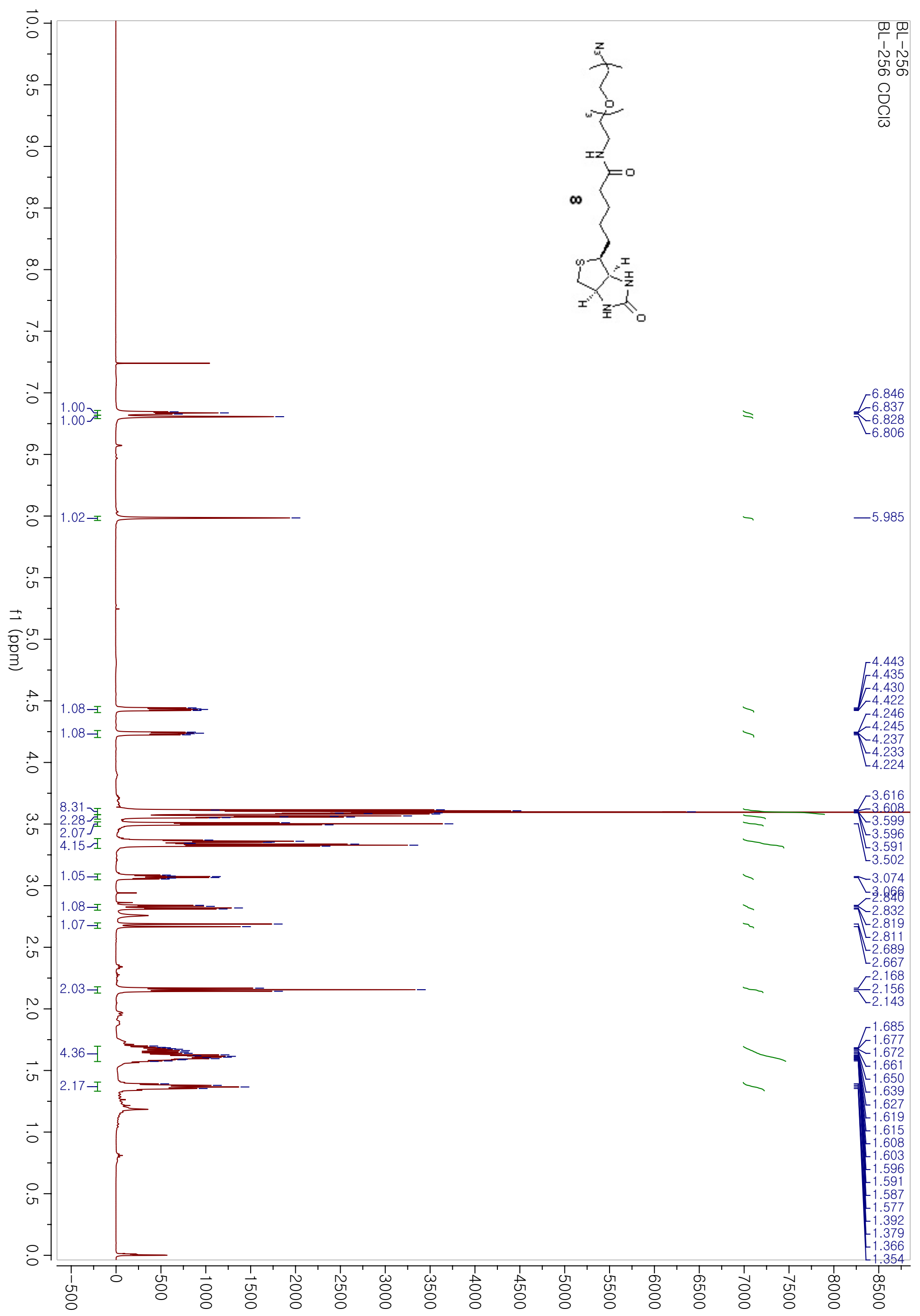




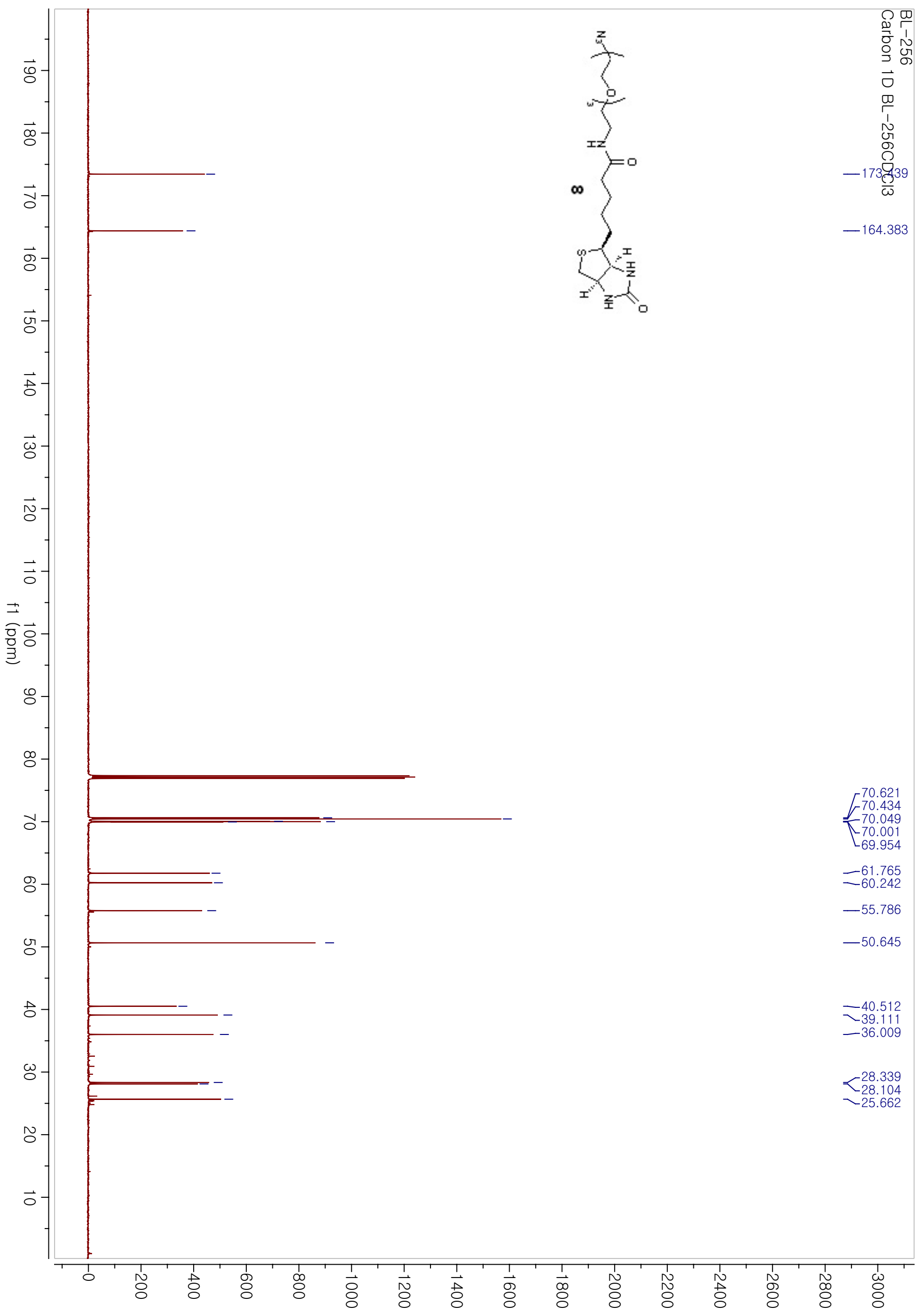




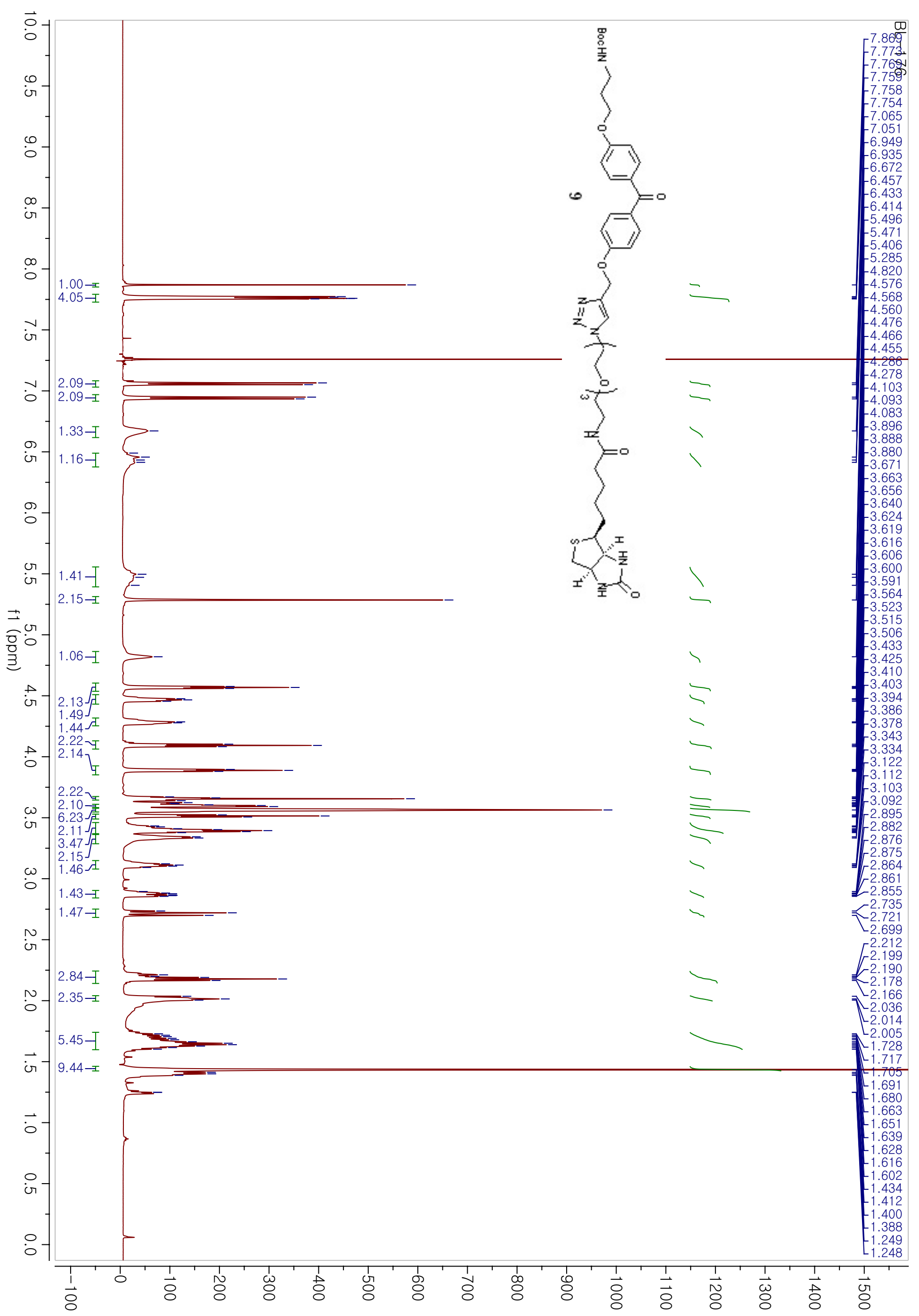




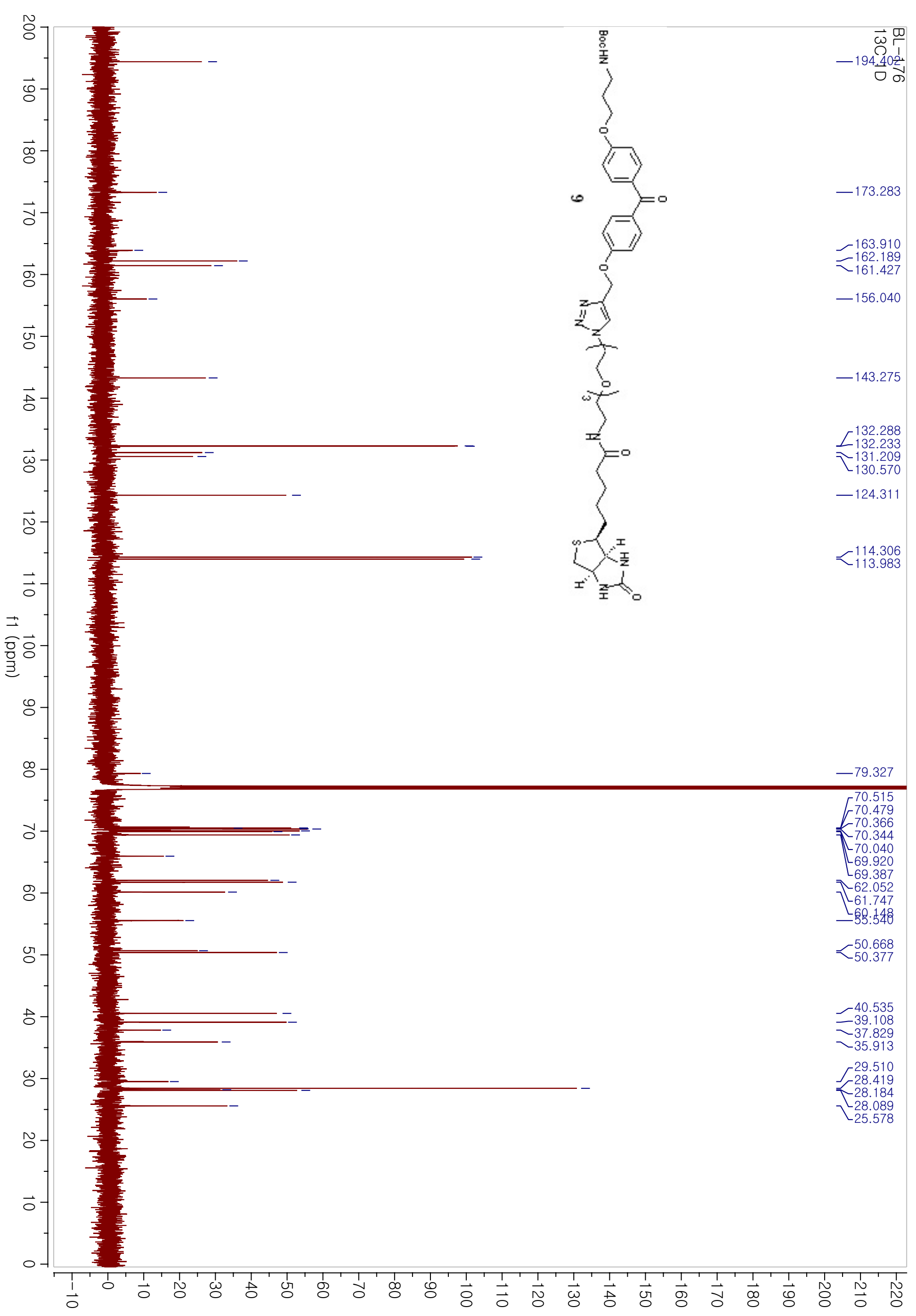




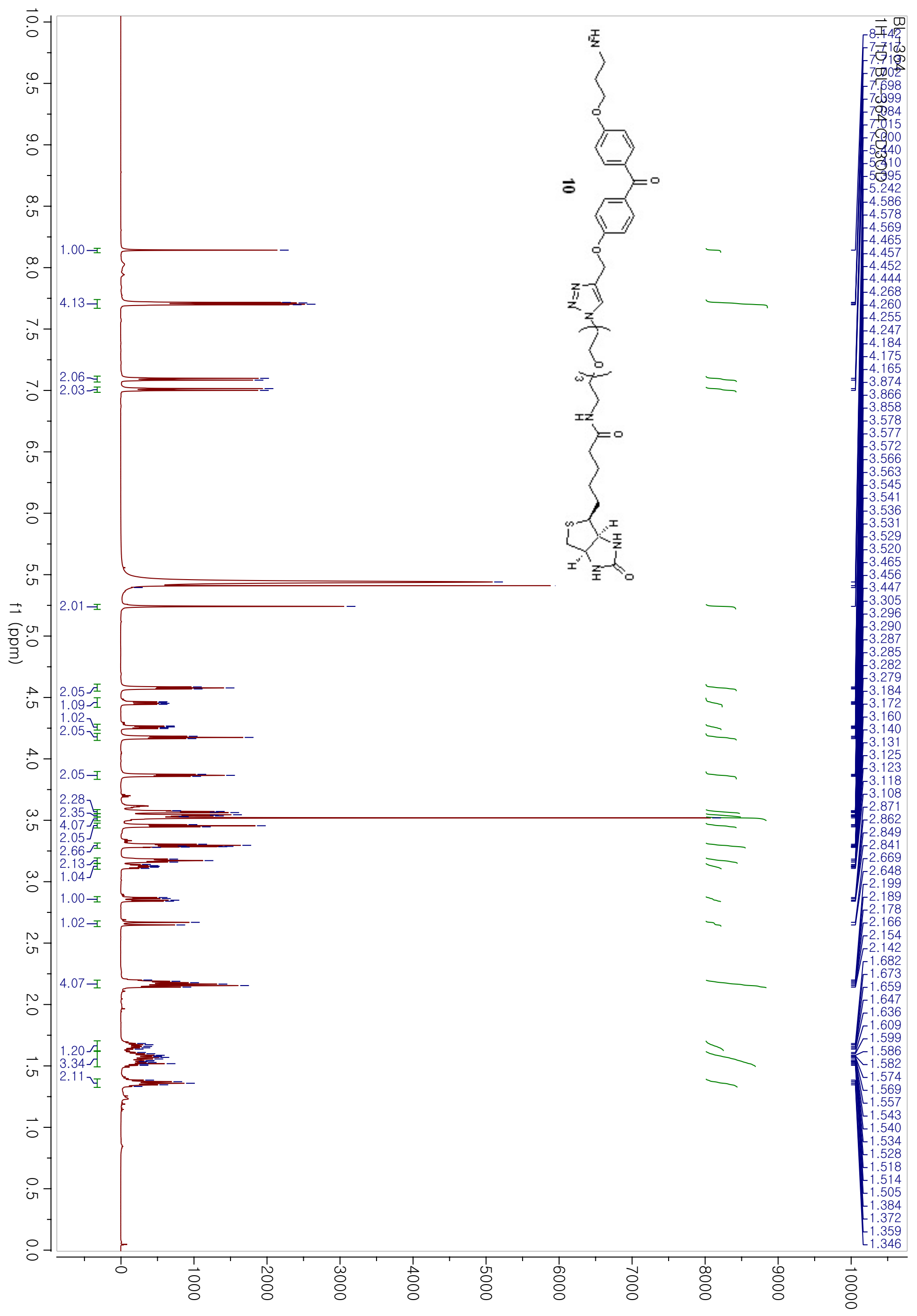




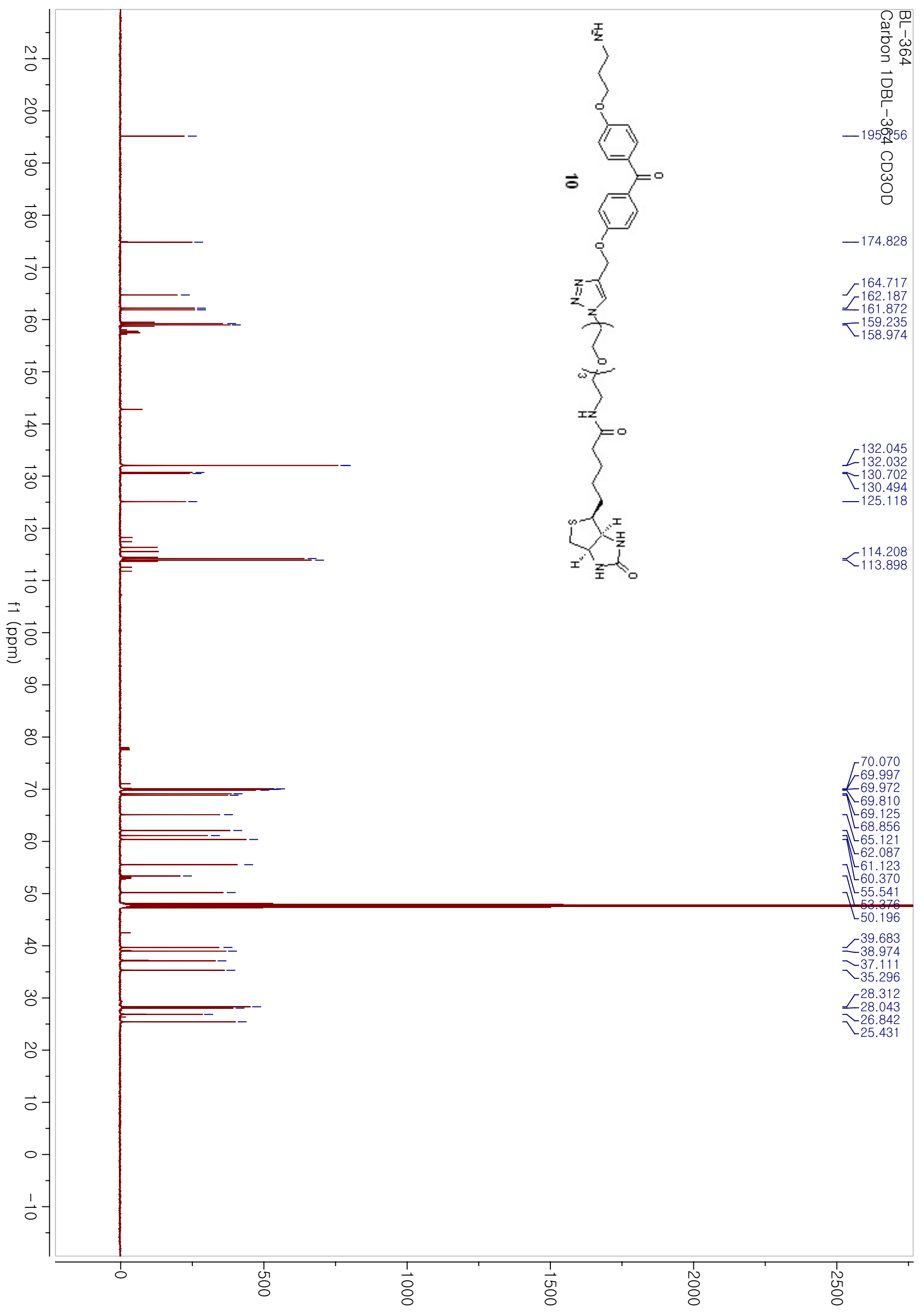




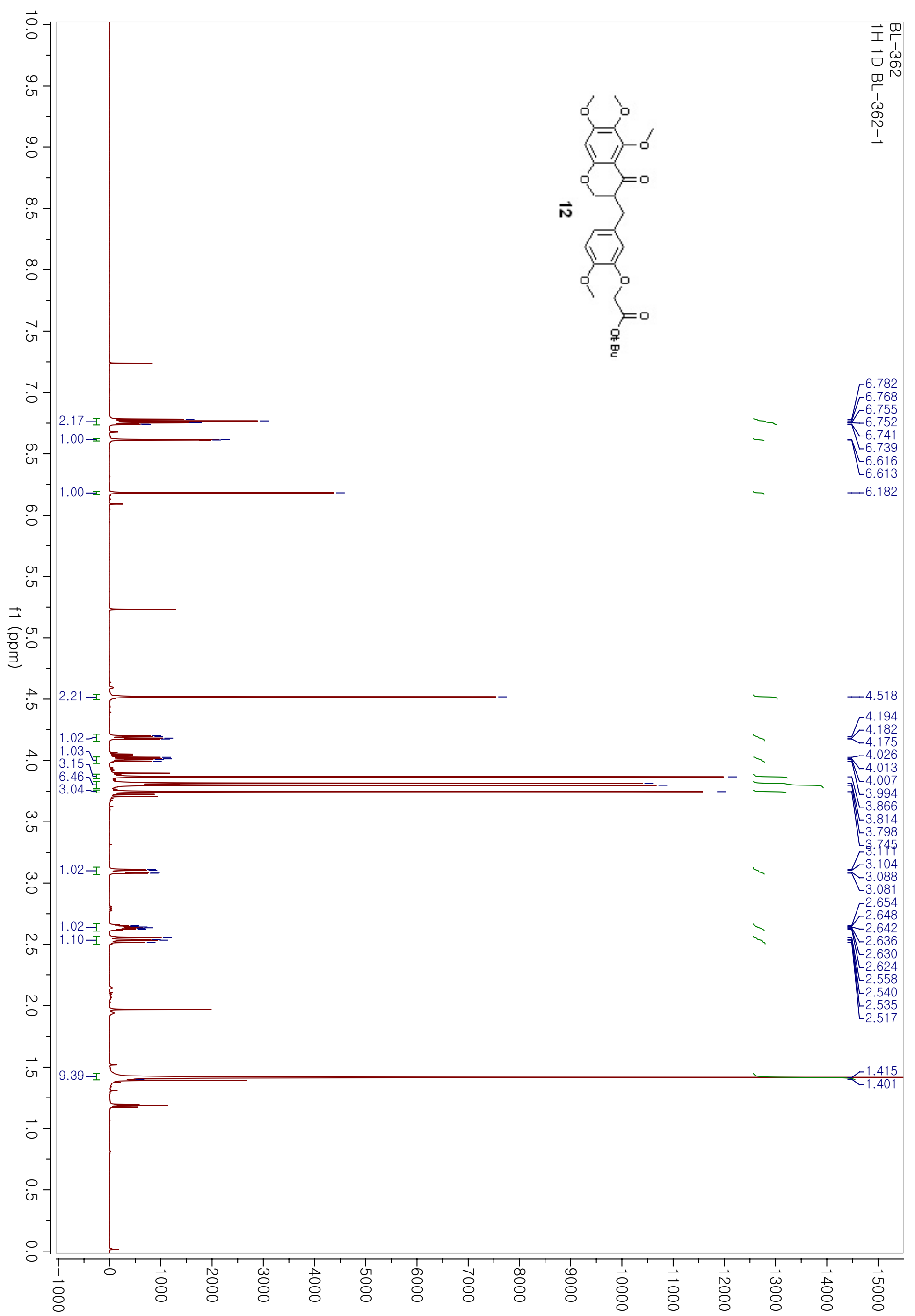




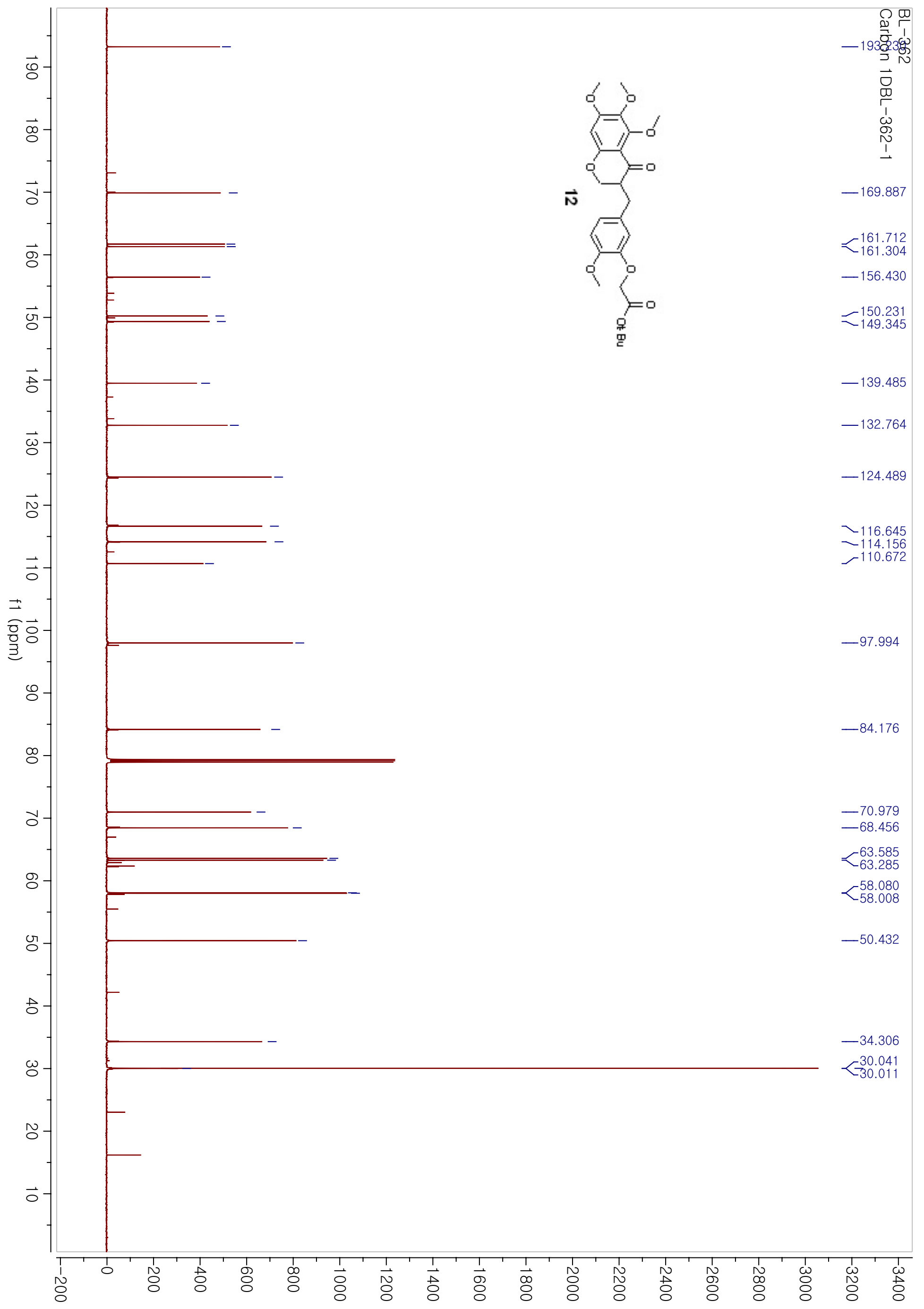




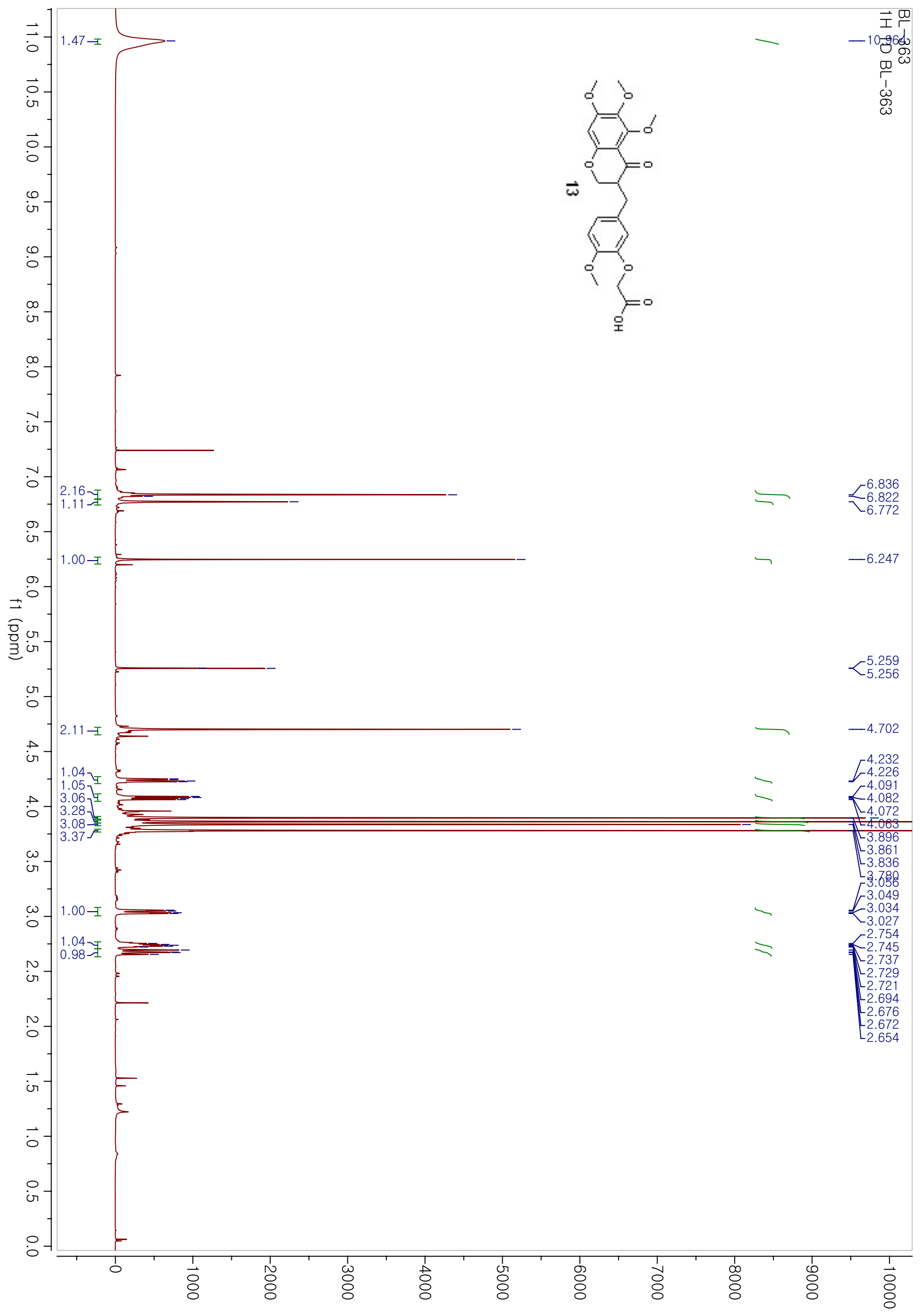




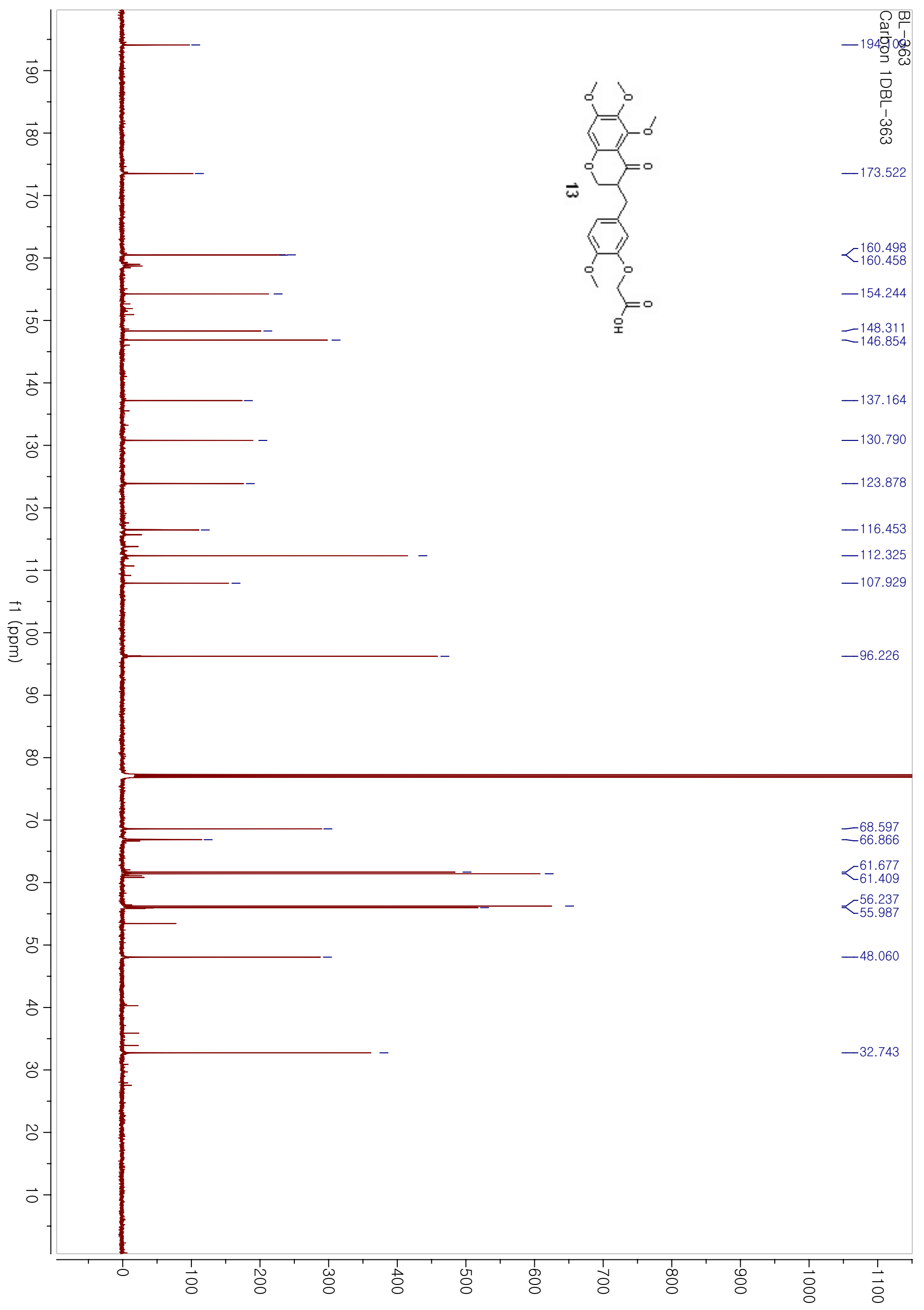




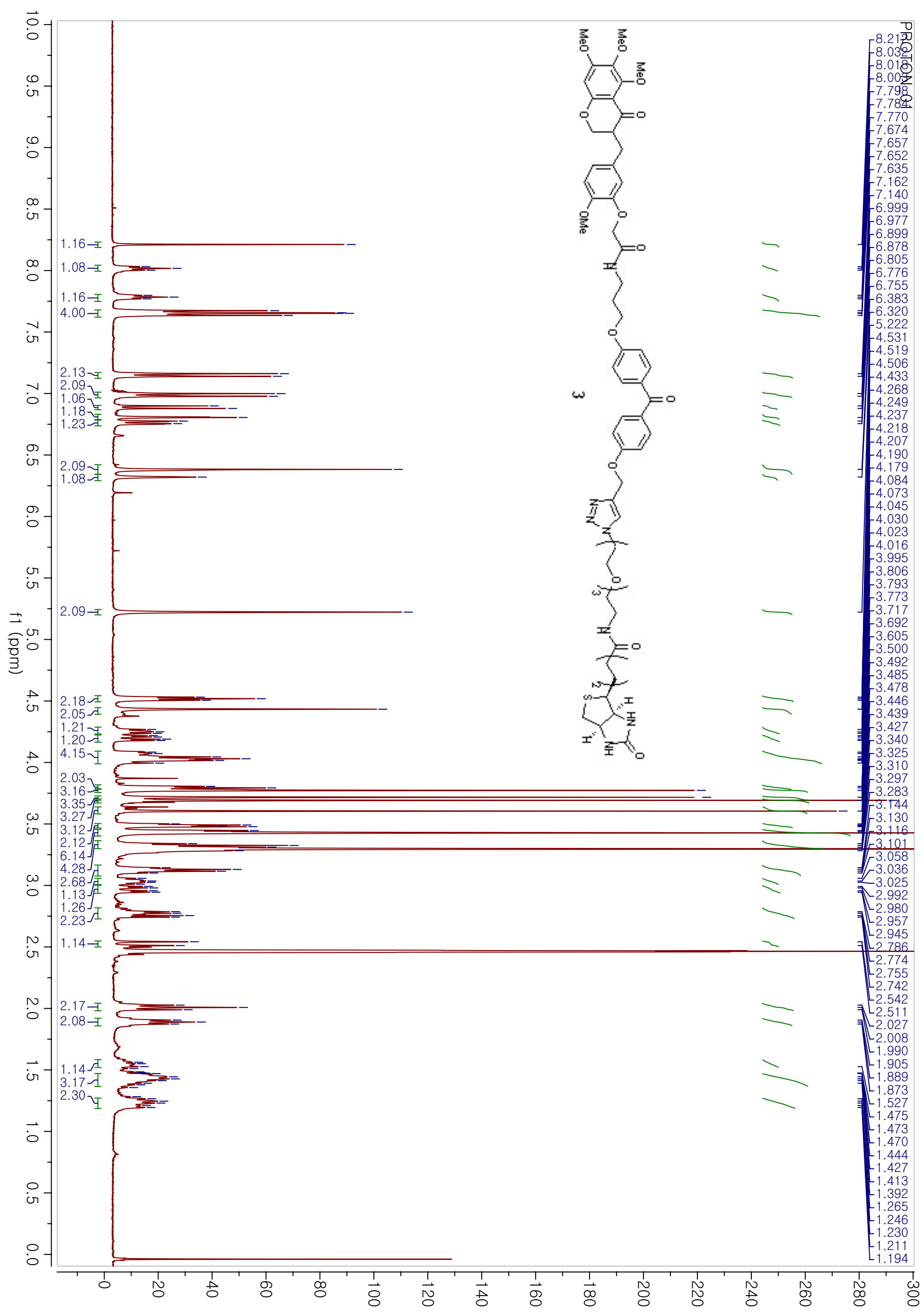




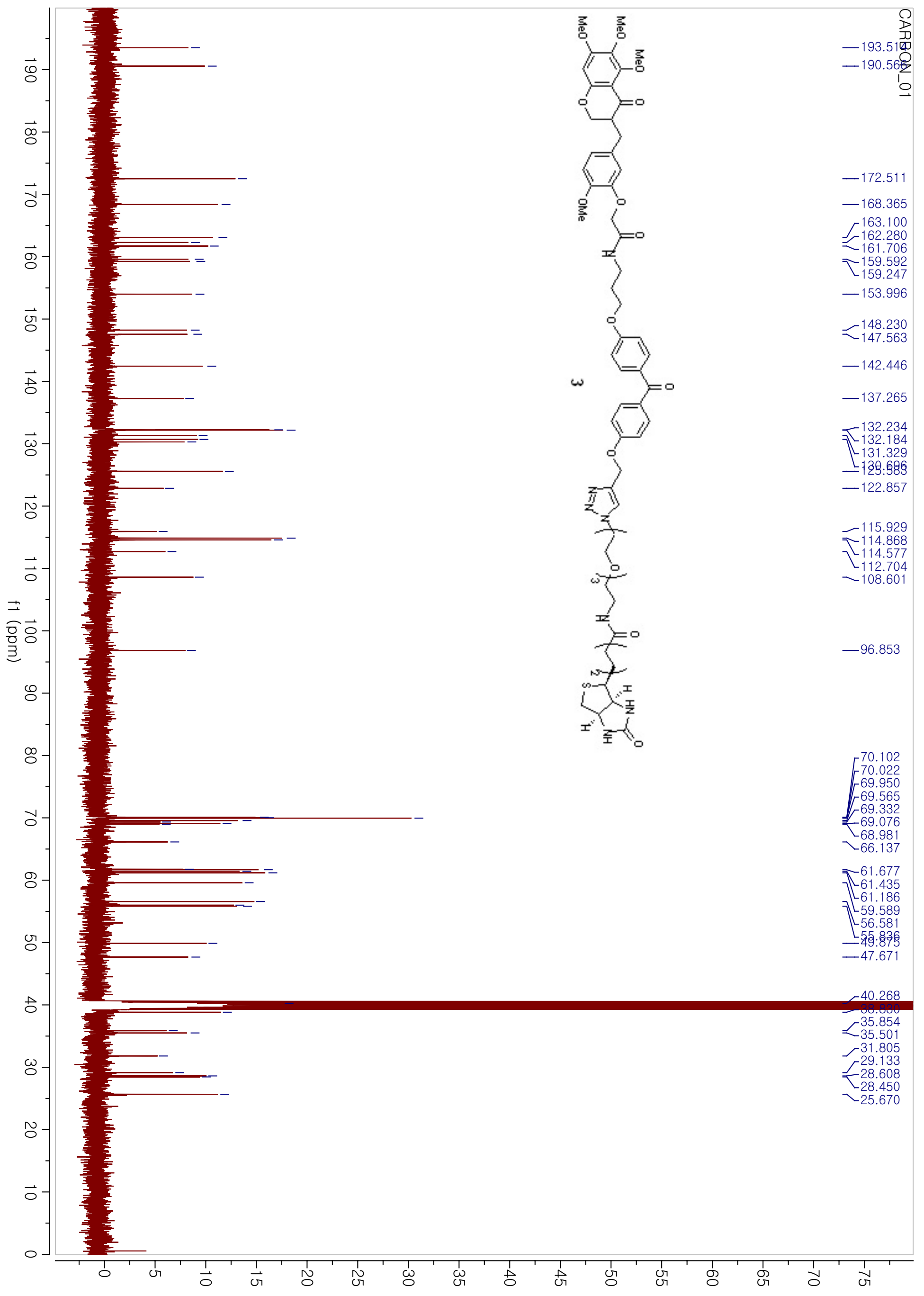




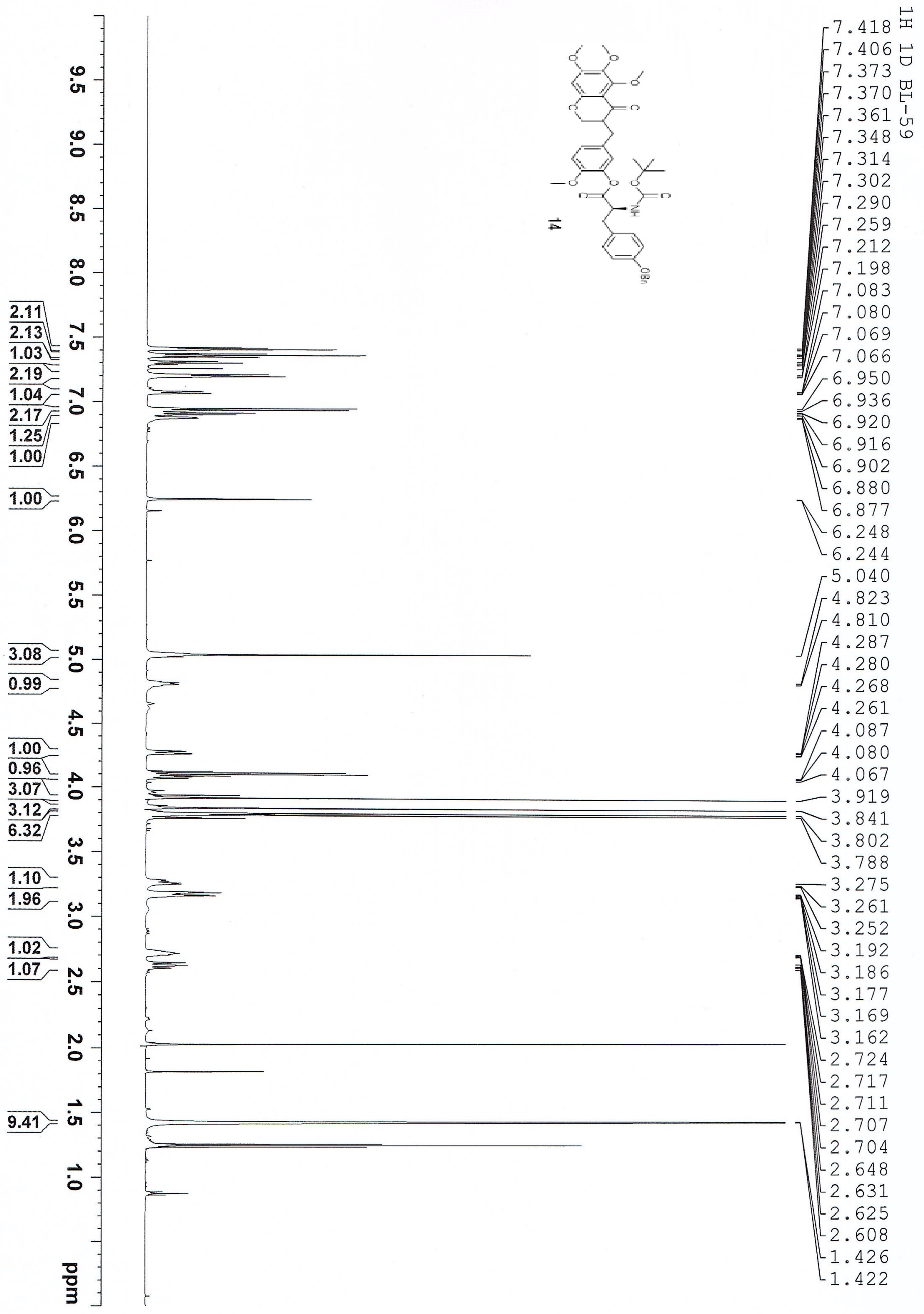




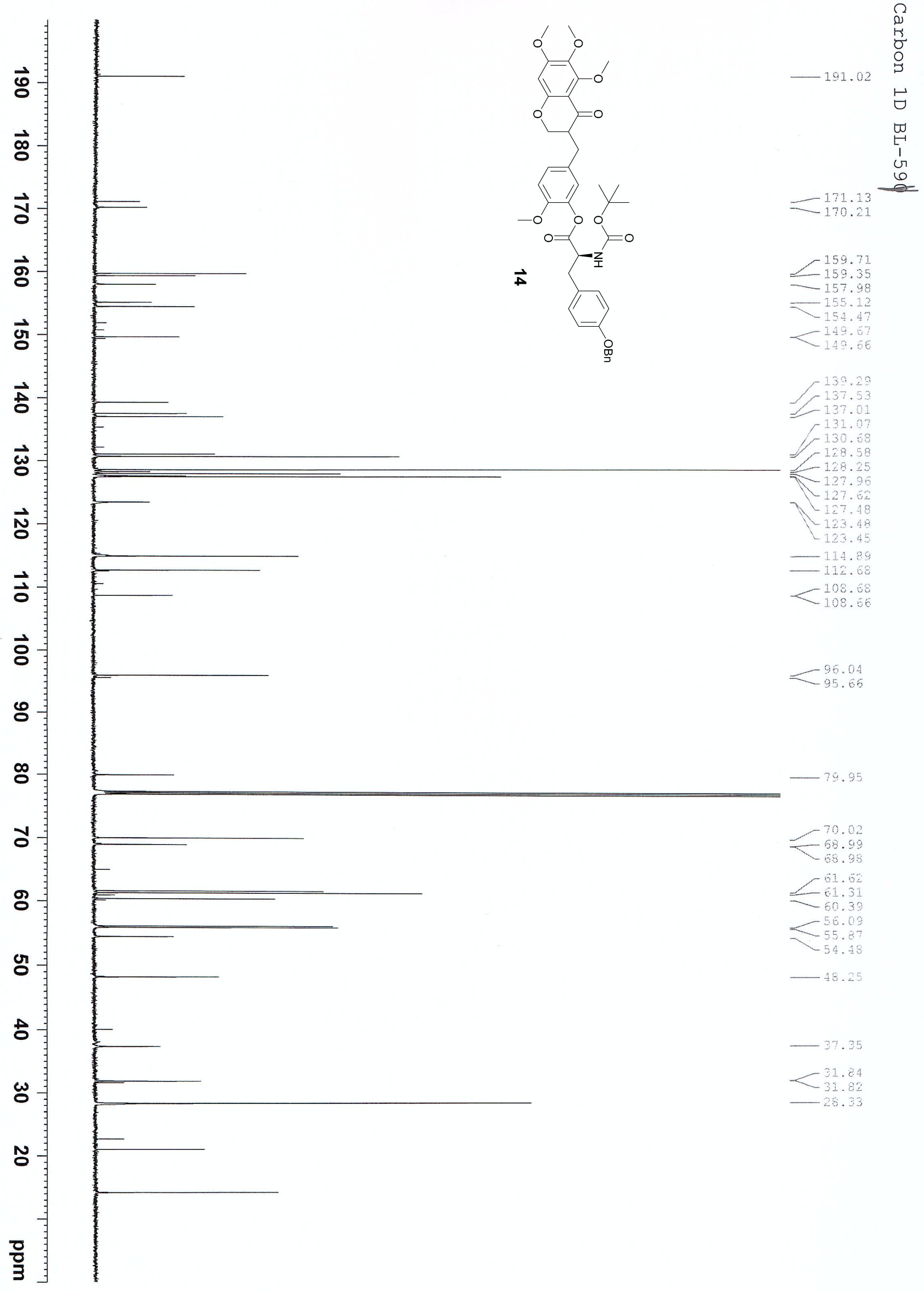




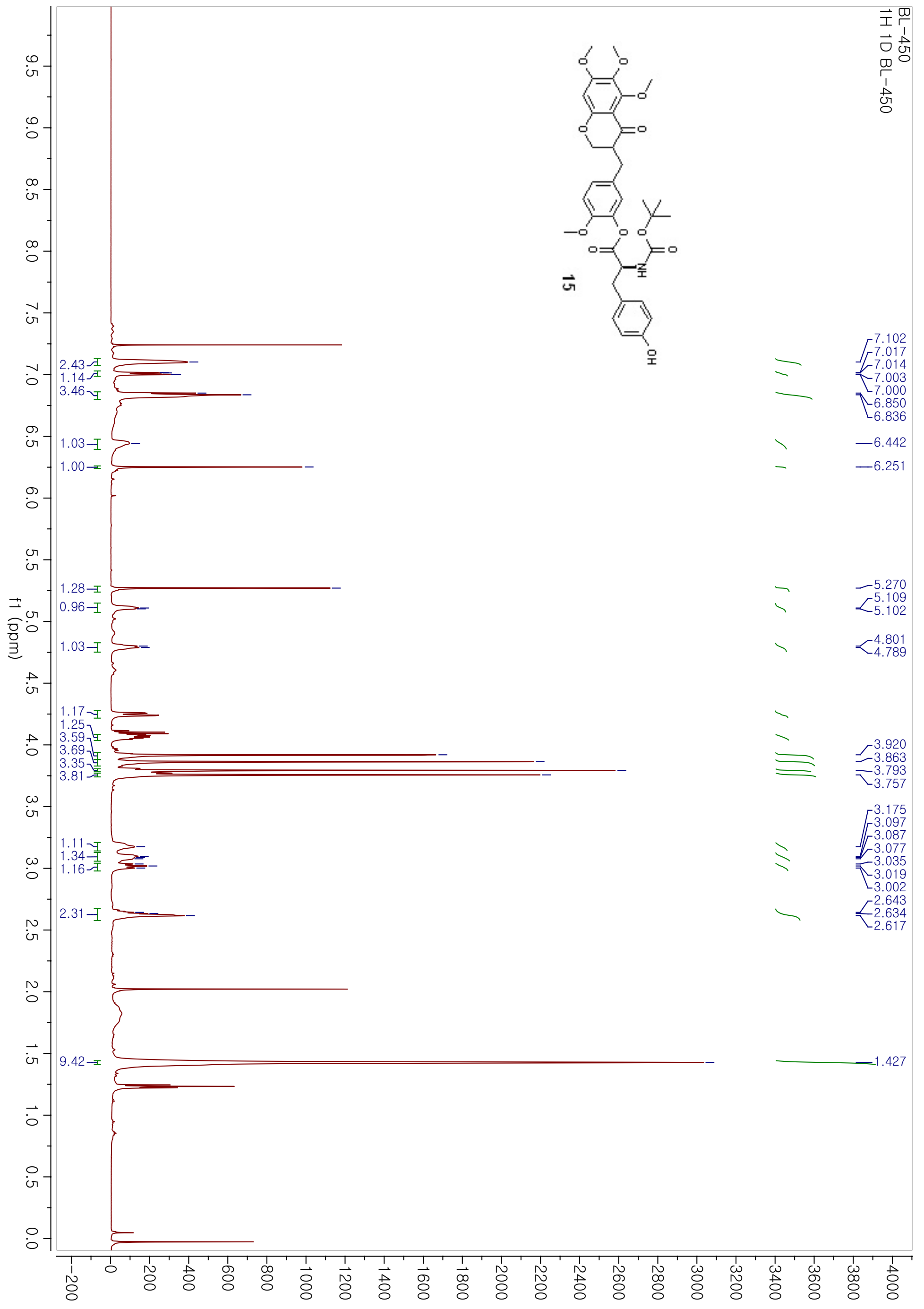




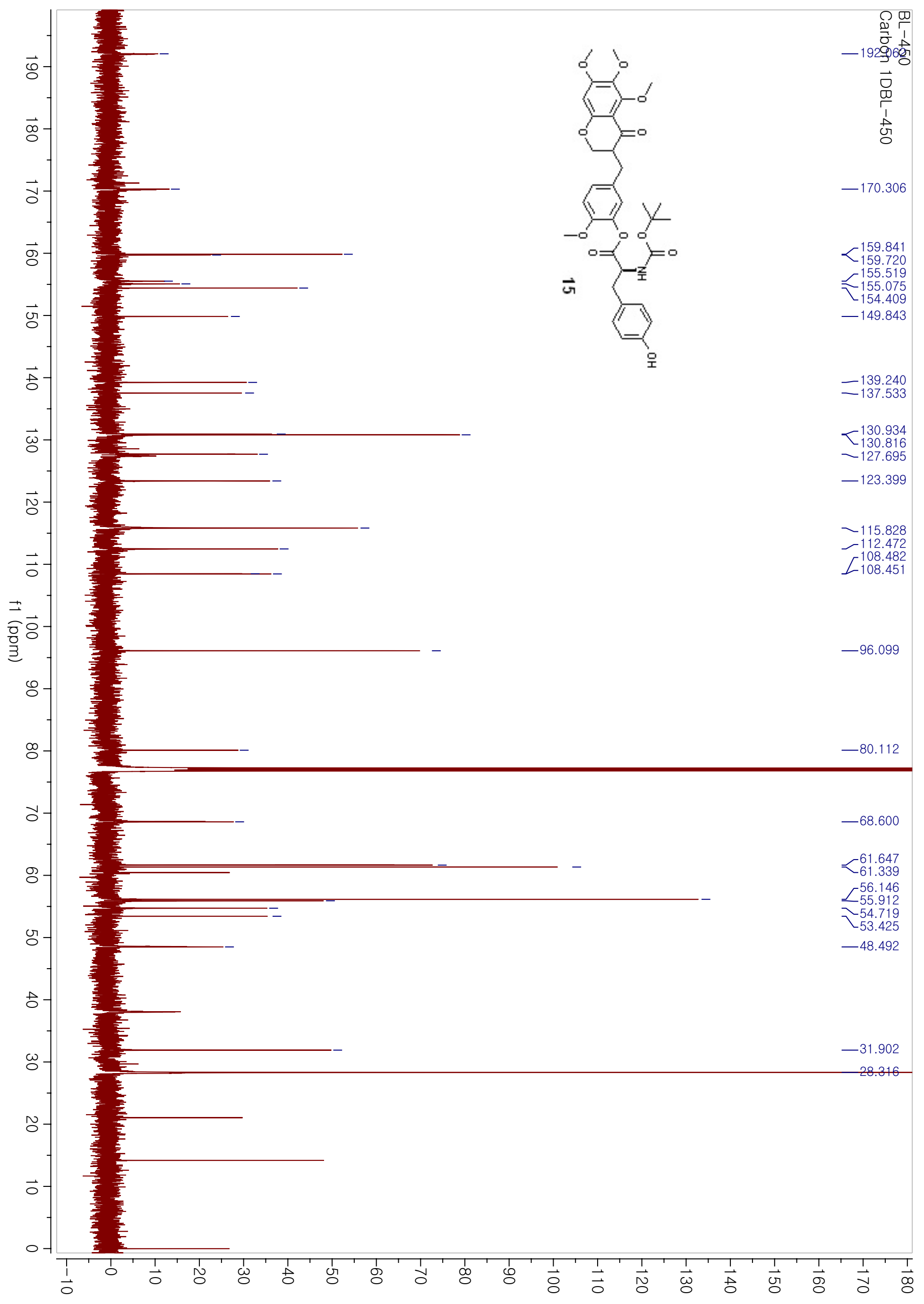




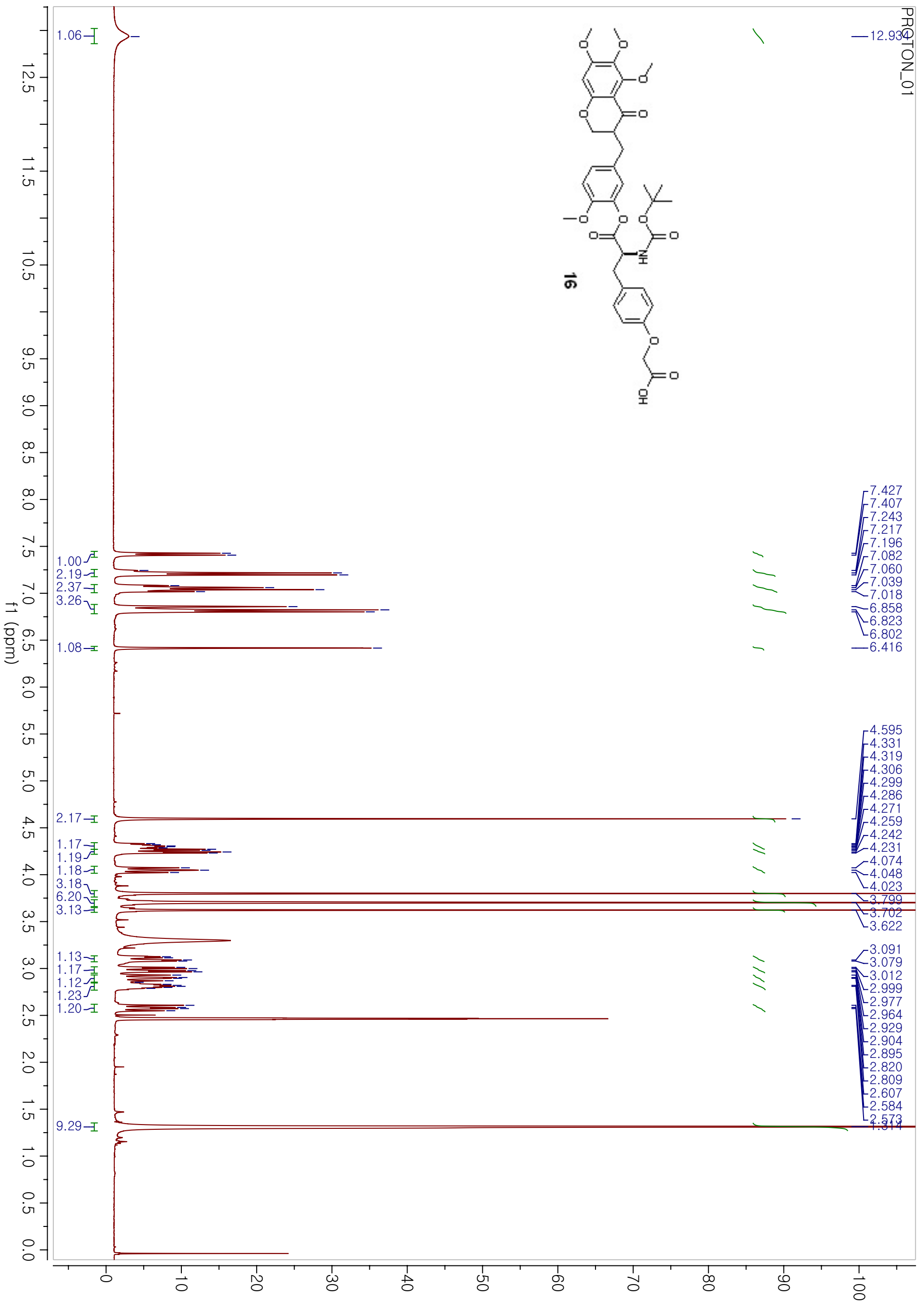




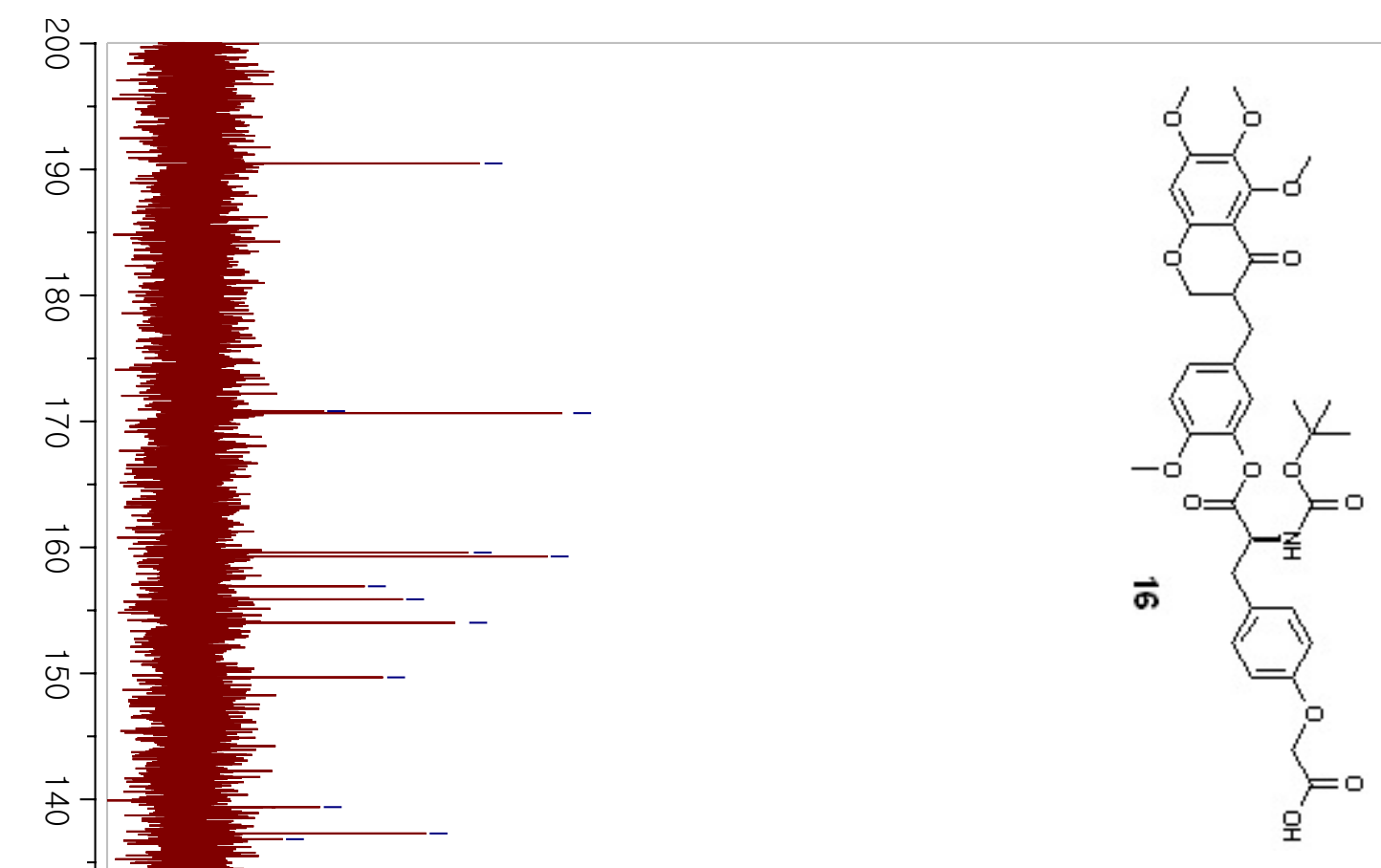

8
3
D
0
-190.2
1
오

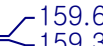

$-156.9$

155.9
-154.0

$-154.0$

$-149.7$

$\vec{\omega}$

흘

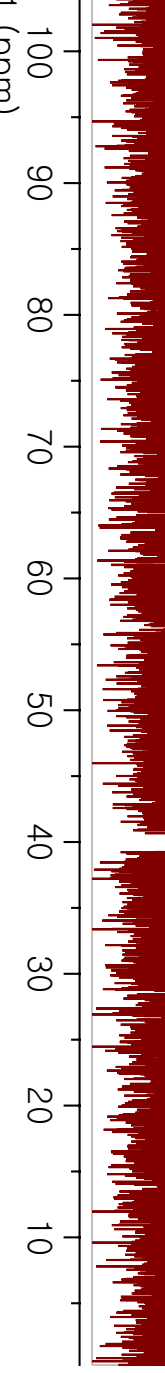

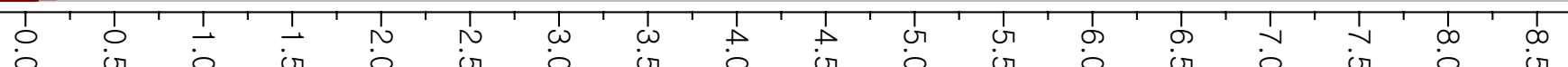




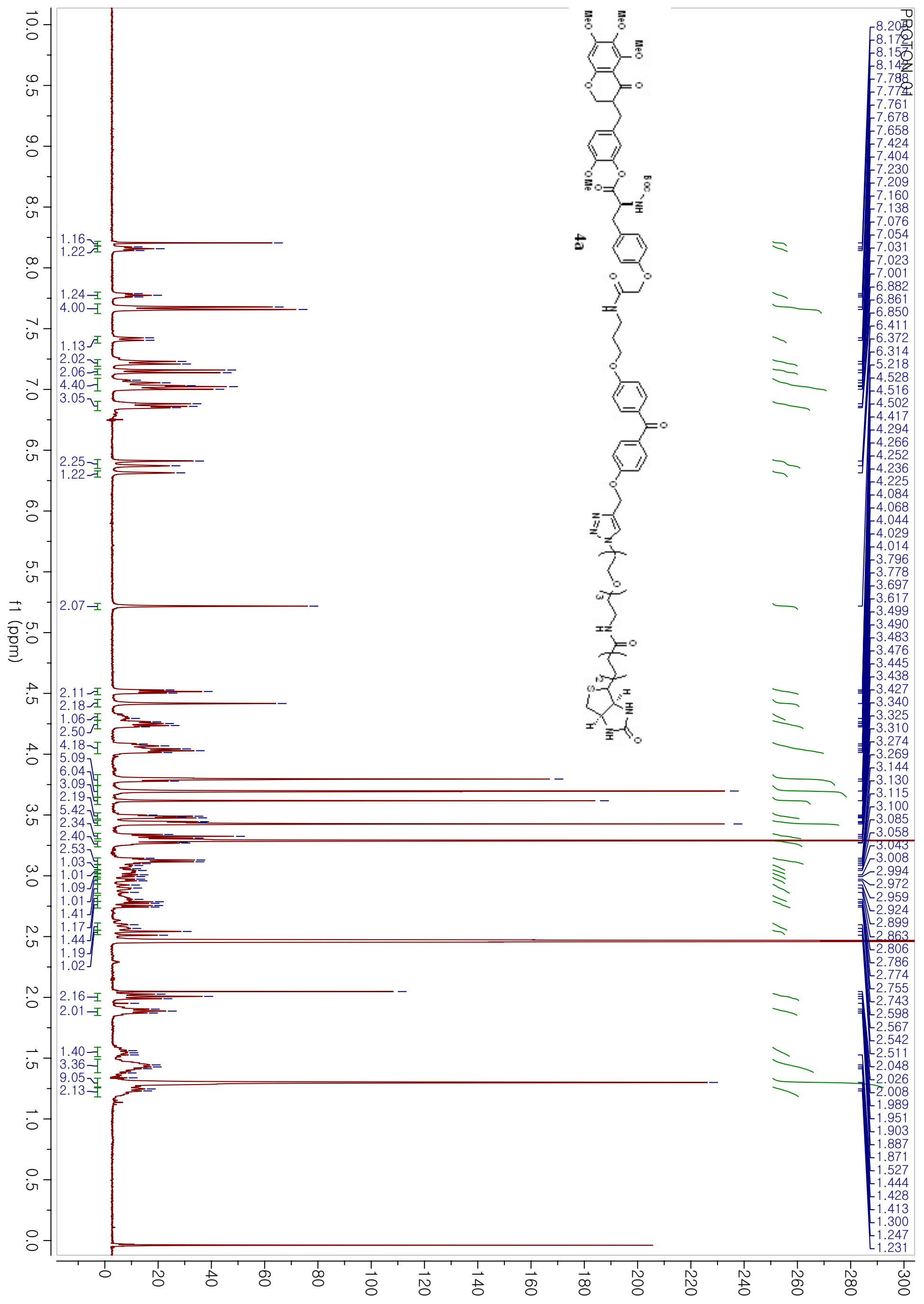




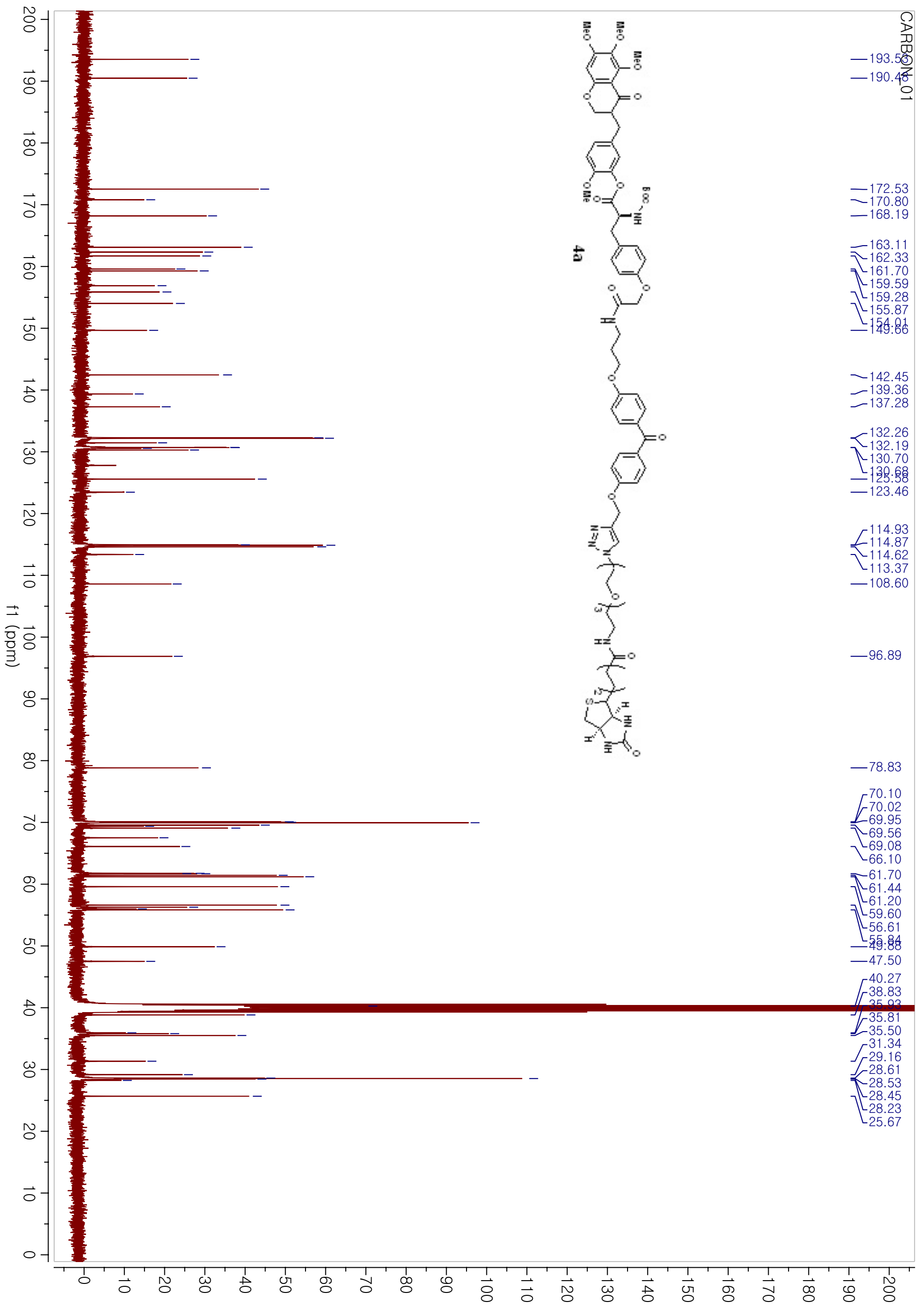




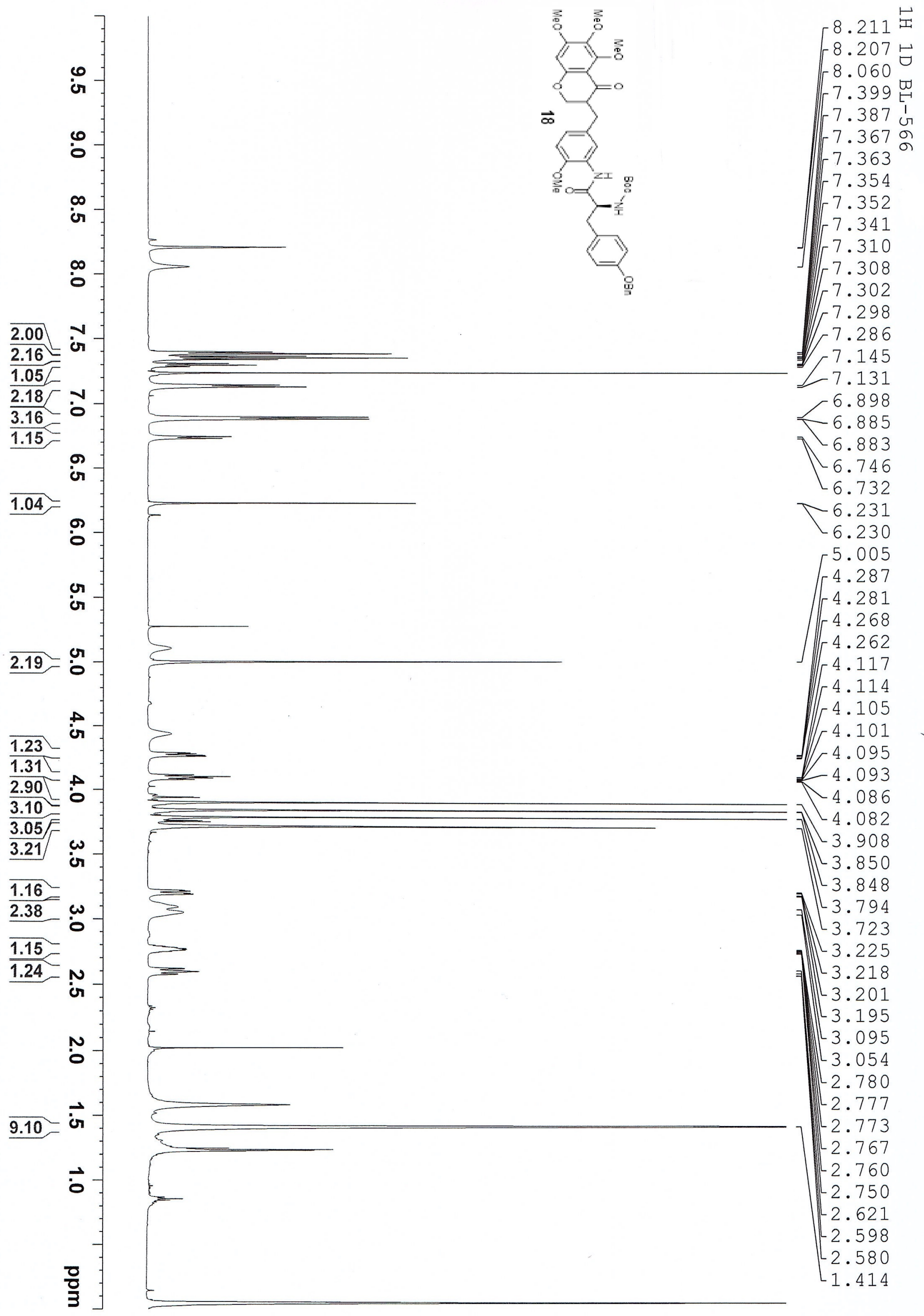




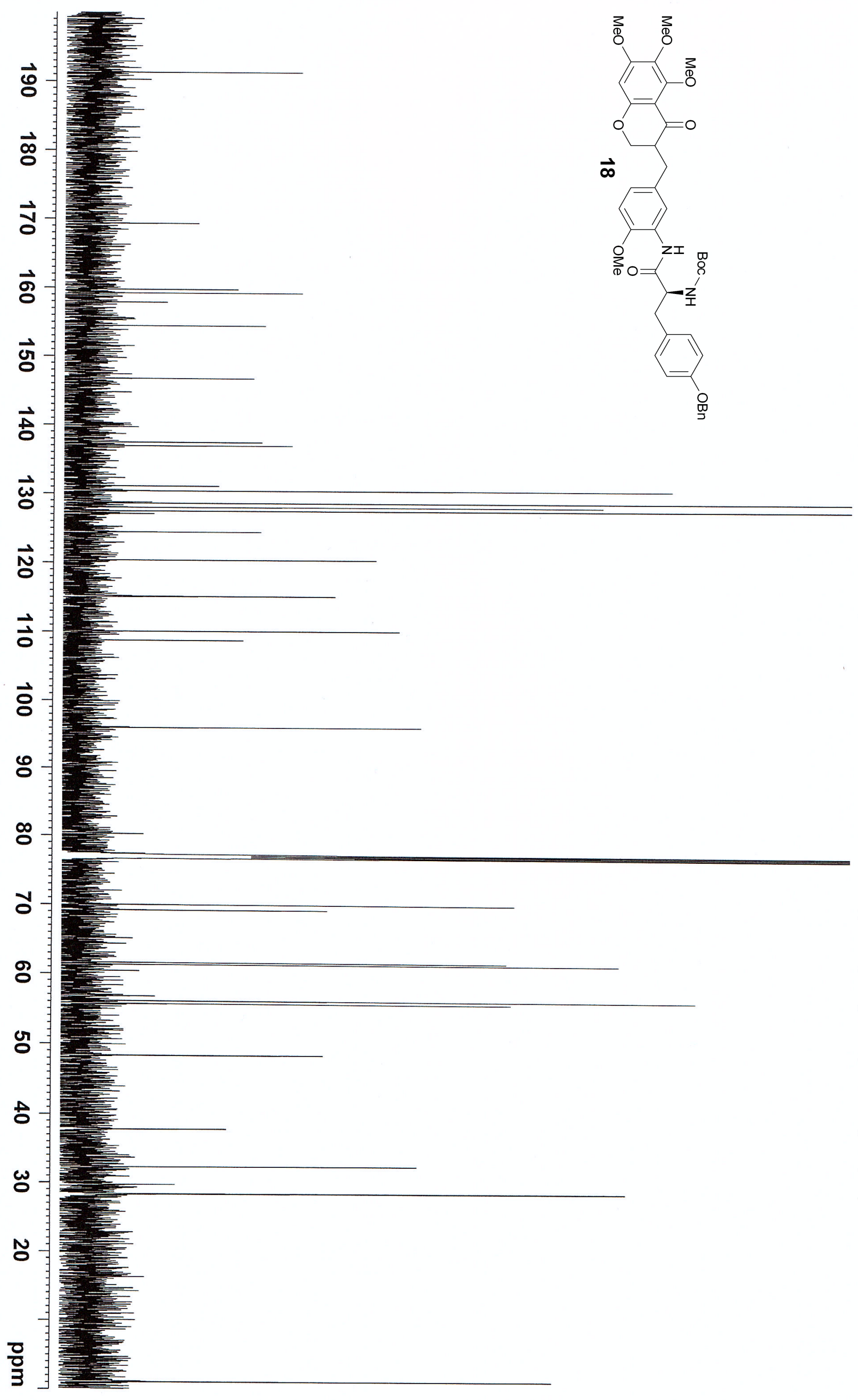




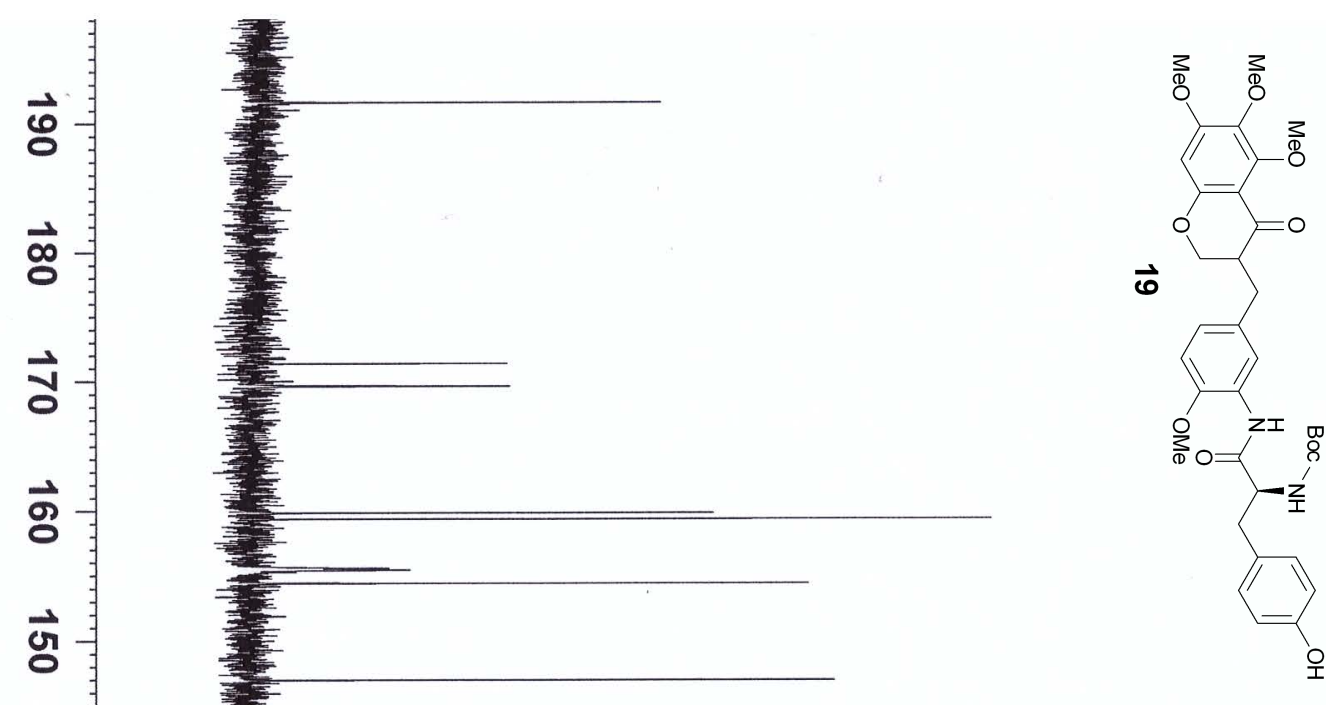

$\overrightarrow{0}$

$\vec{\omega}$

$\overrightarrow{0}$

$\overrightarrow{0}$

웅

$\dddot{\circ}$

$\stackrel{\circ}{\circ}$

ฮै

용

동

t

๘

ก

몰

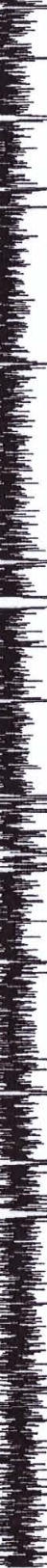




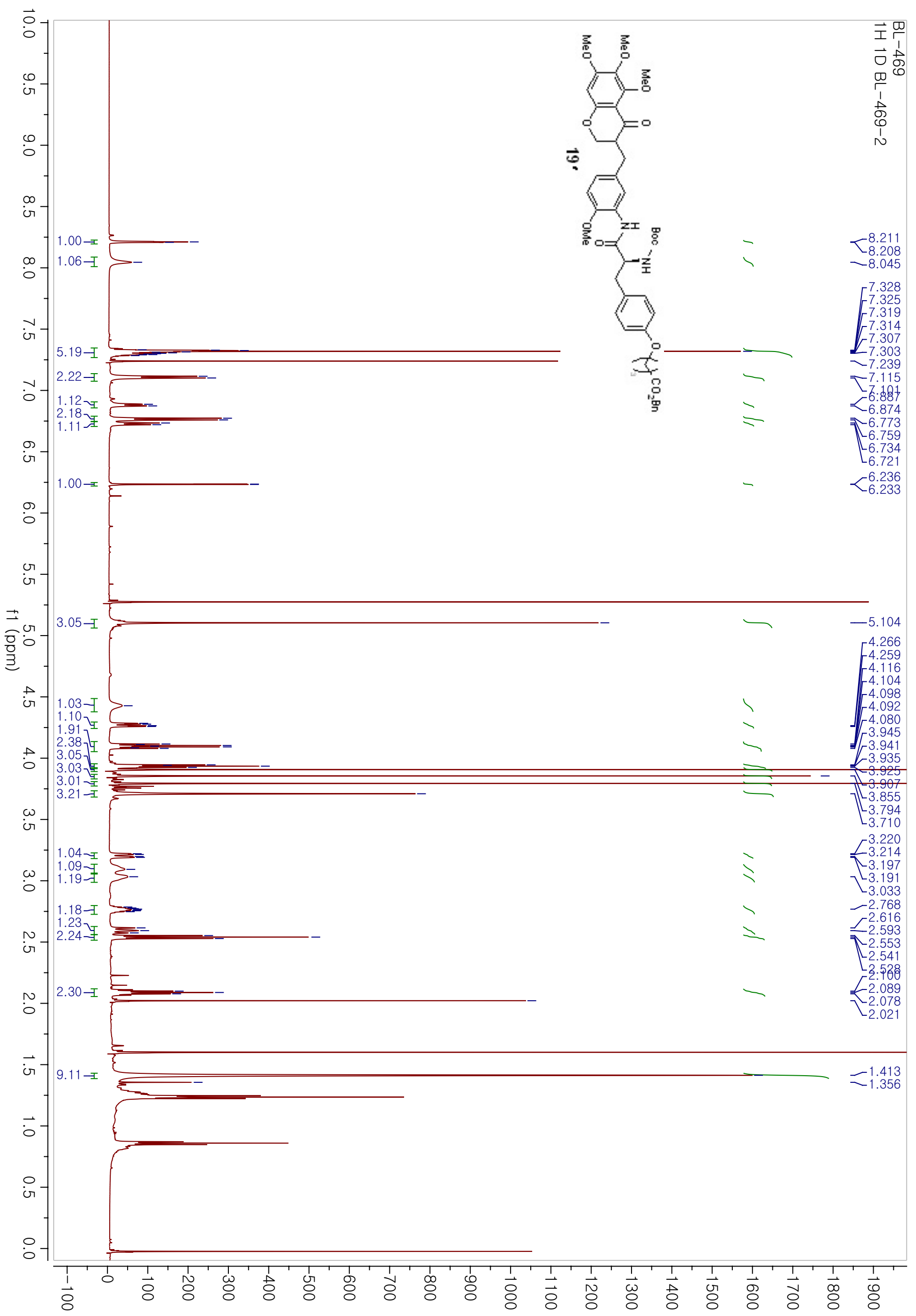




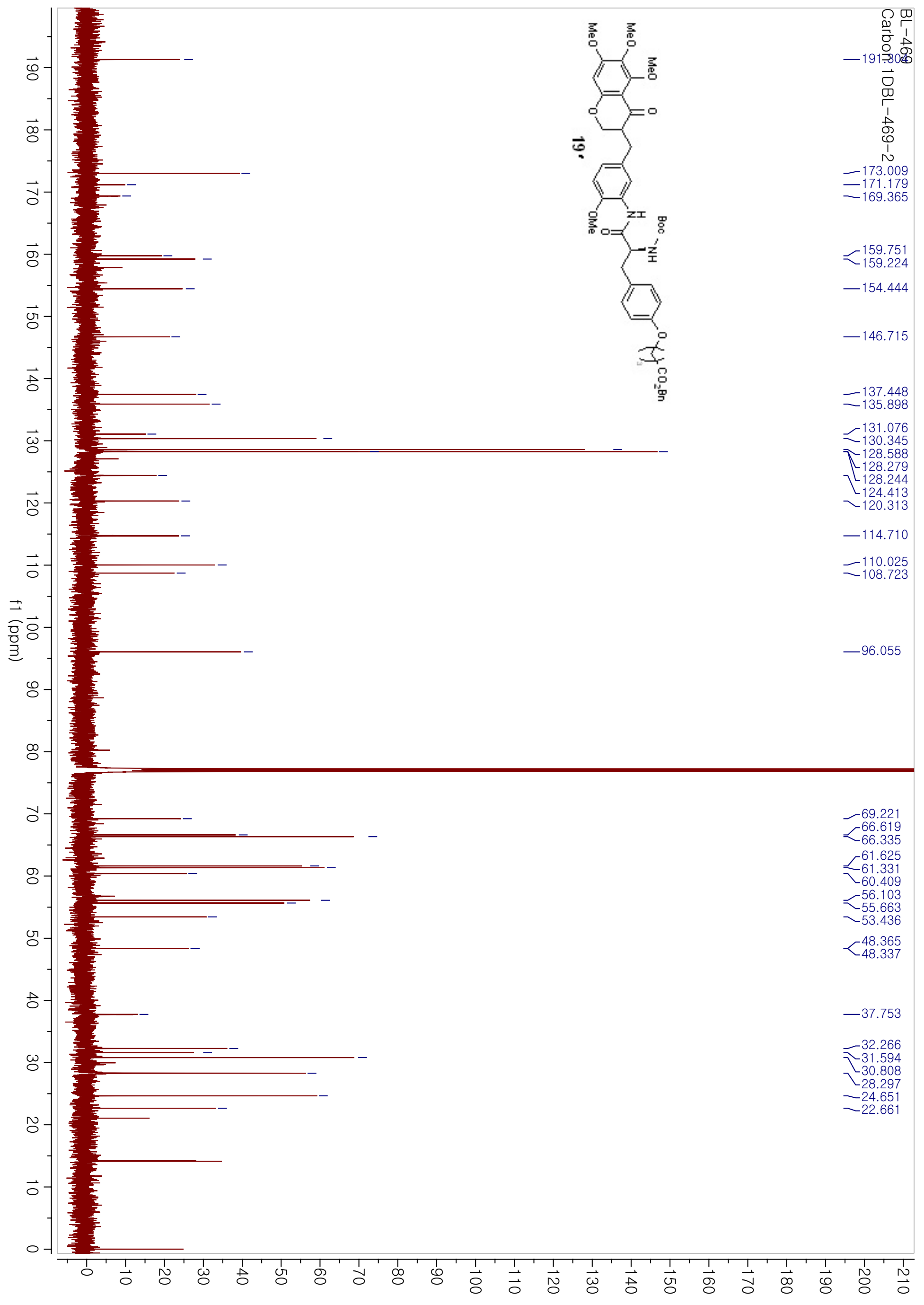




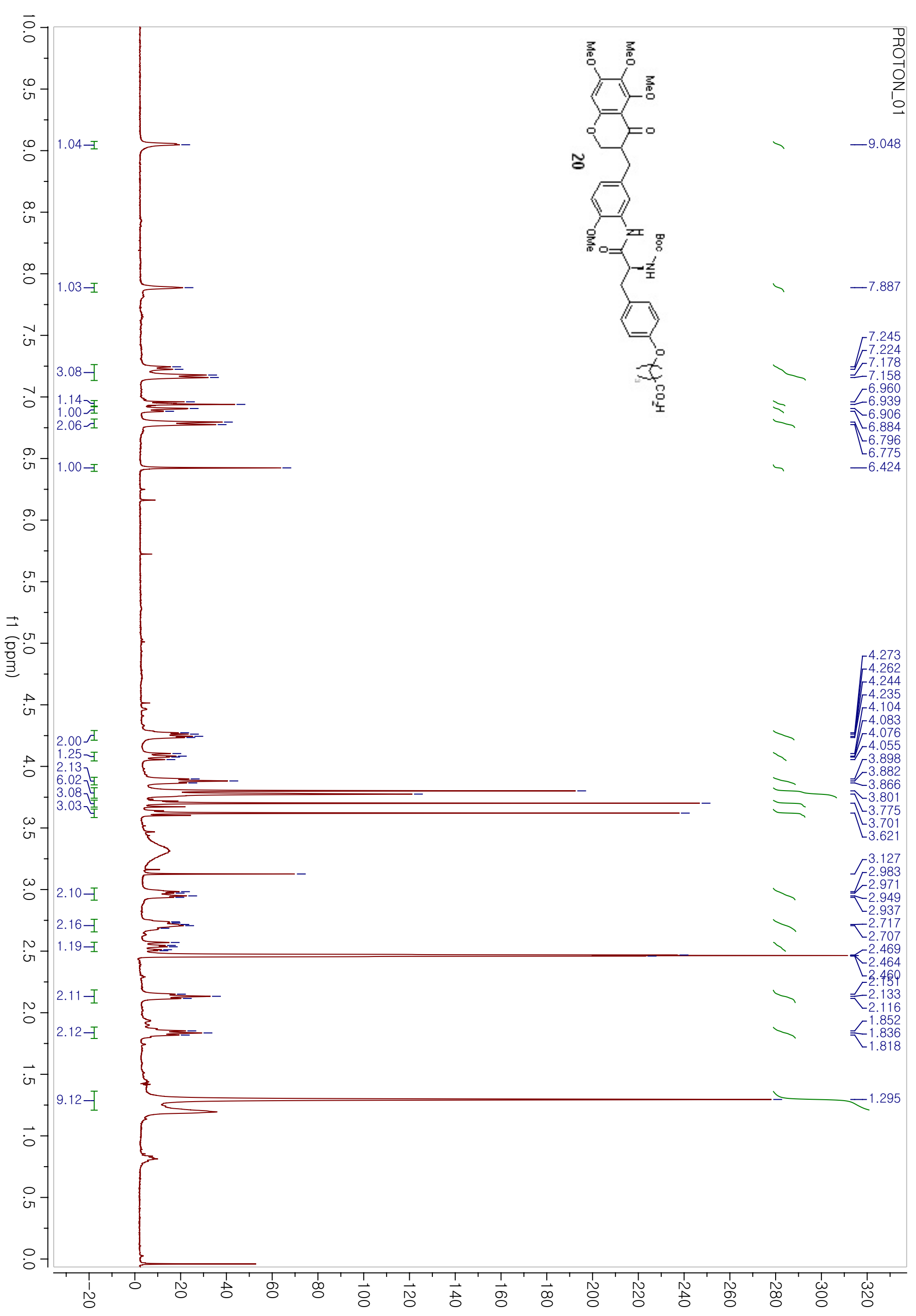




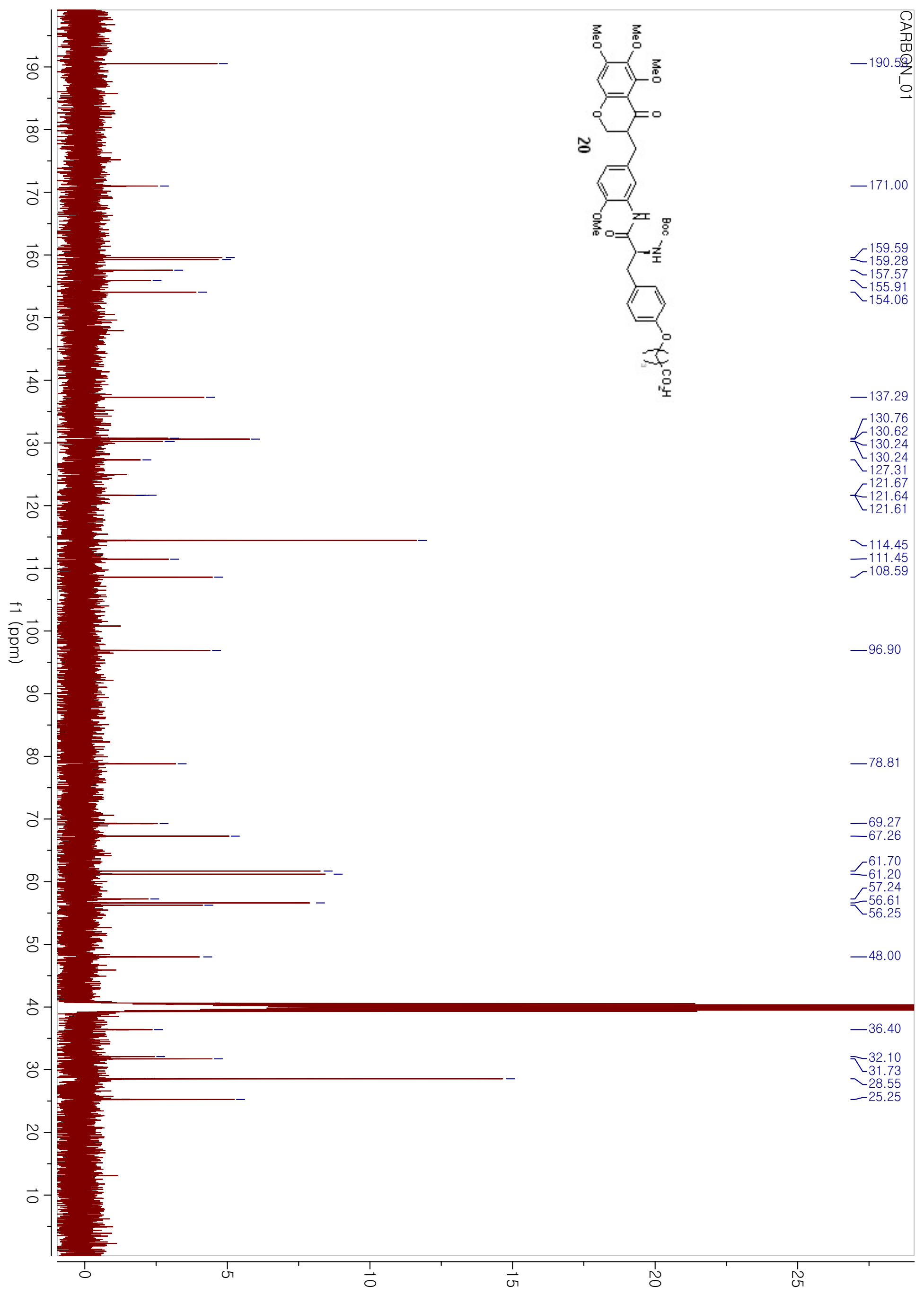




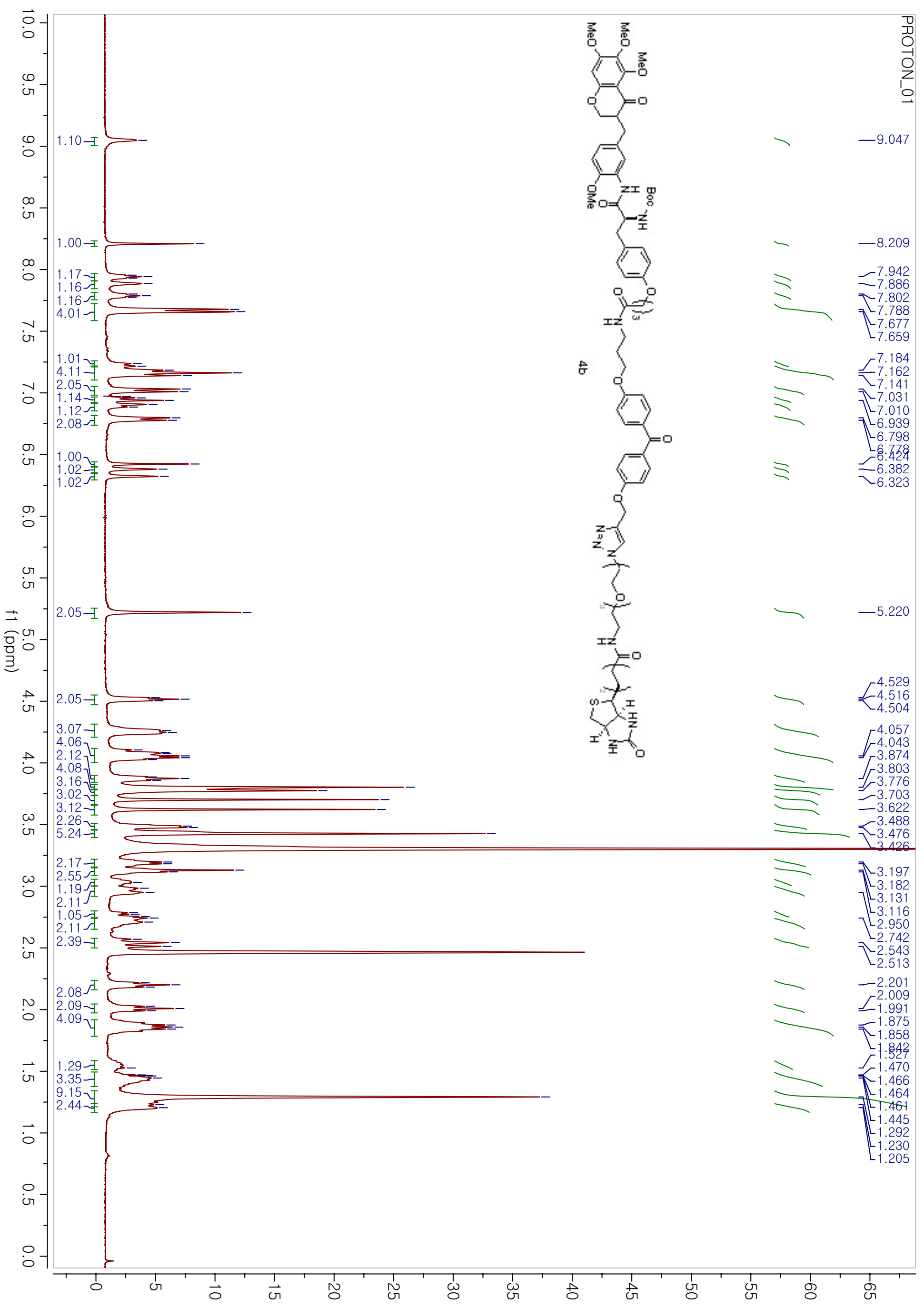




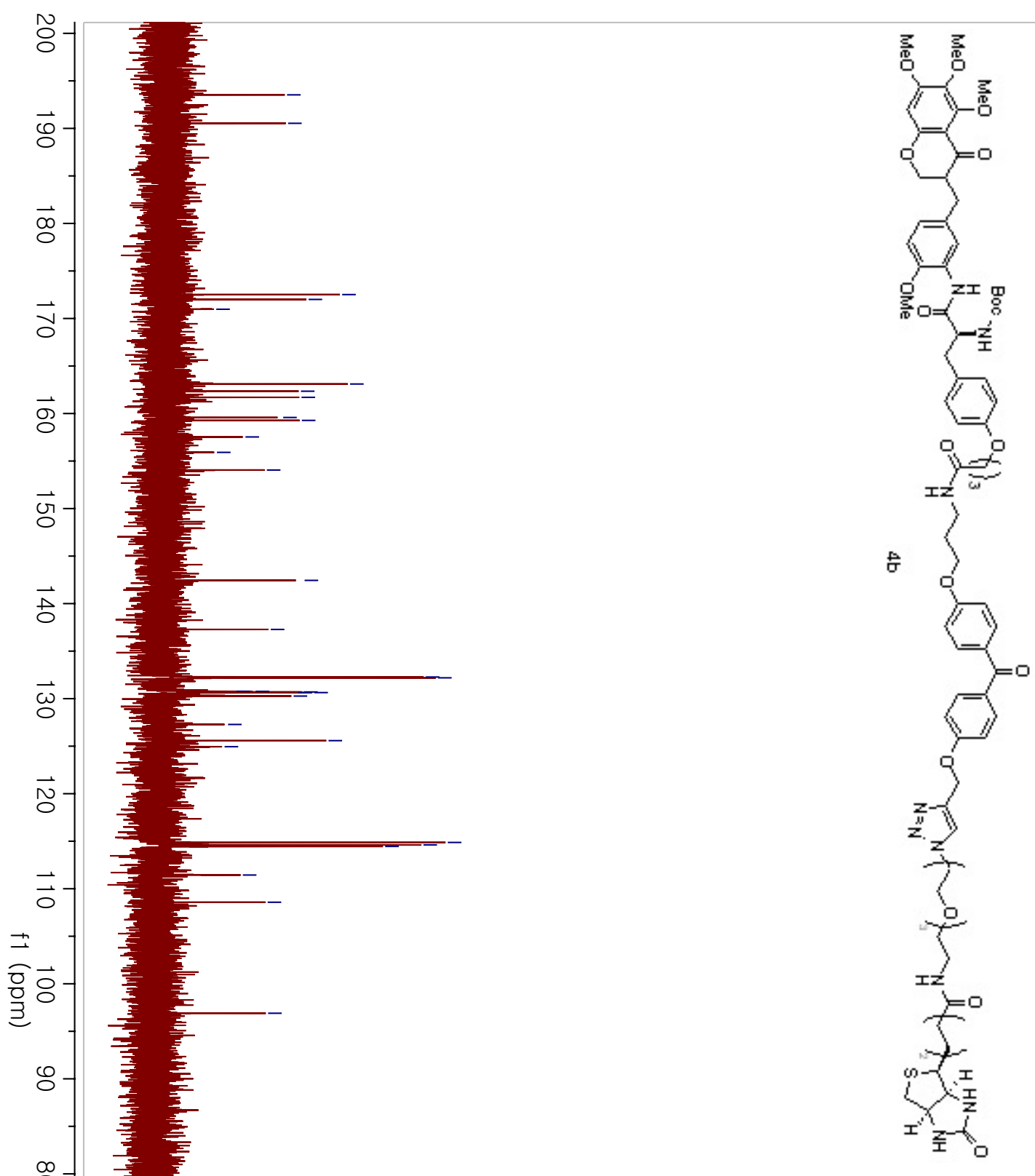

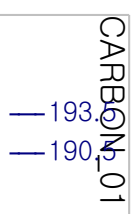

172.5
-172.0

$-171.0$

163.1

$-161.7$

$\tau^{159.6}$

$-157.5$

$+155.9$

$-142.4$

$-137.3$

132.3
-132.2

$-130.8$

130.8

$-130.7$

$-130.6$

130.3

125.9

$-114.6$

$-114.5$

$-108.6$

$-96.9$

$-78.8$

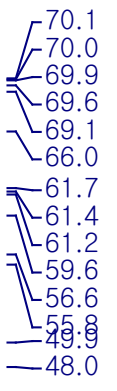

38.8

음

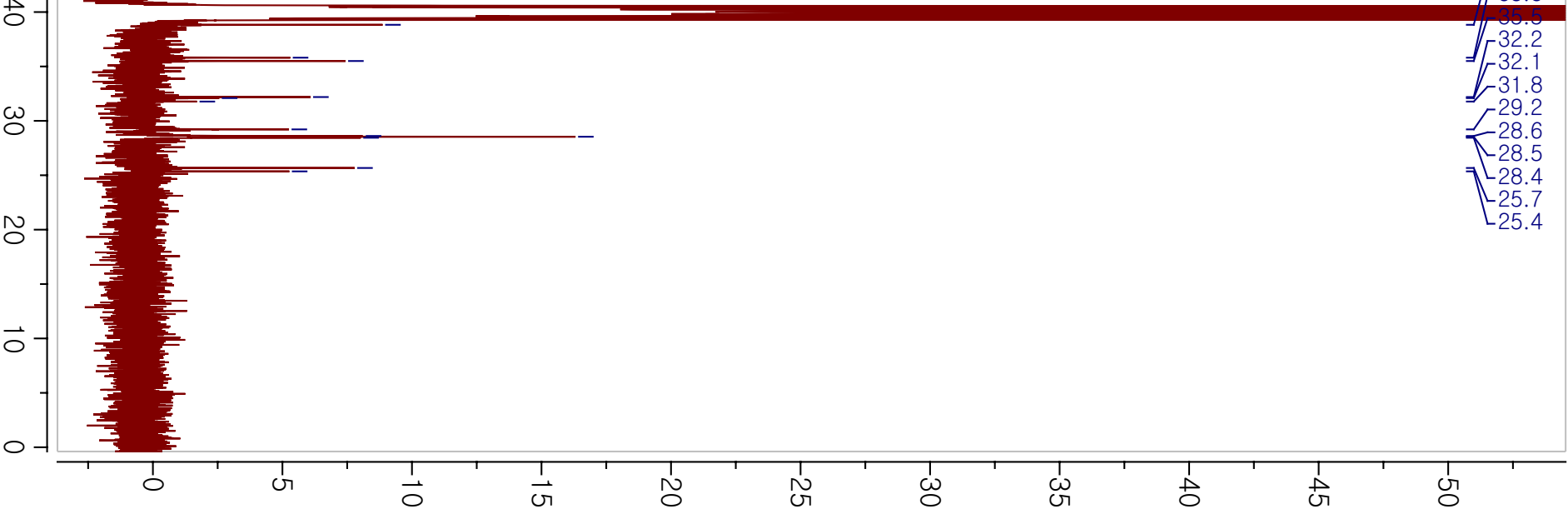




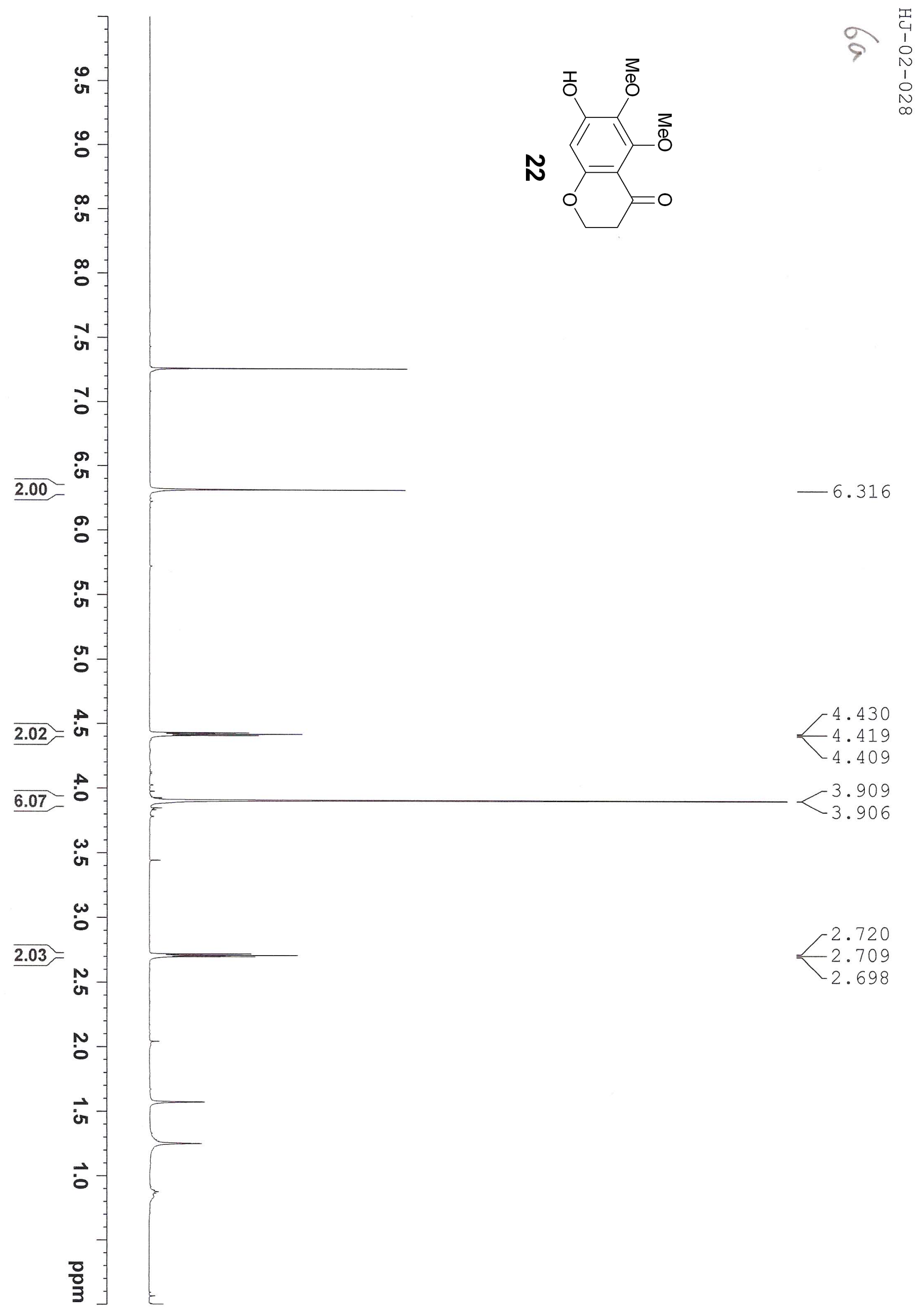




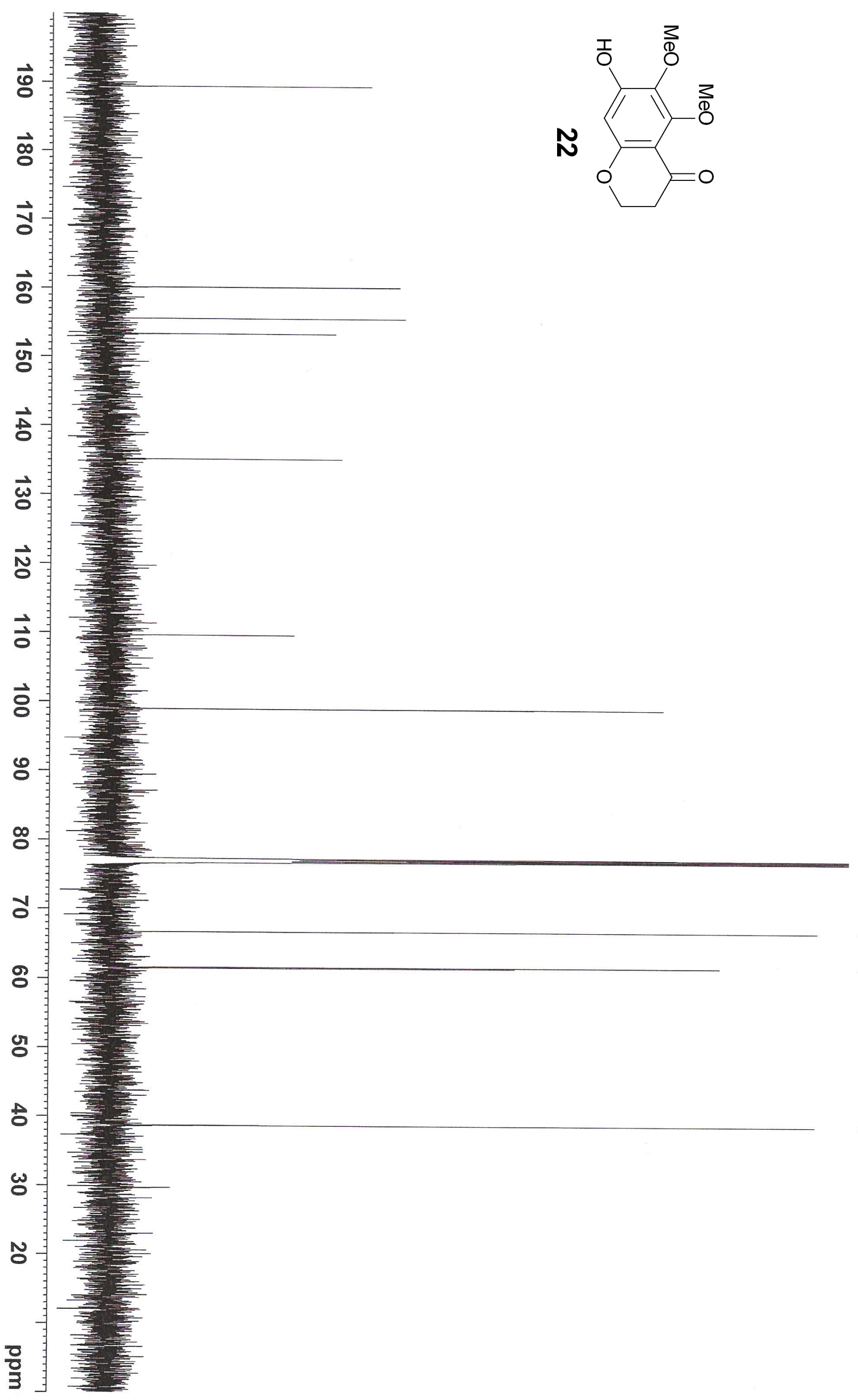




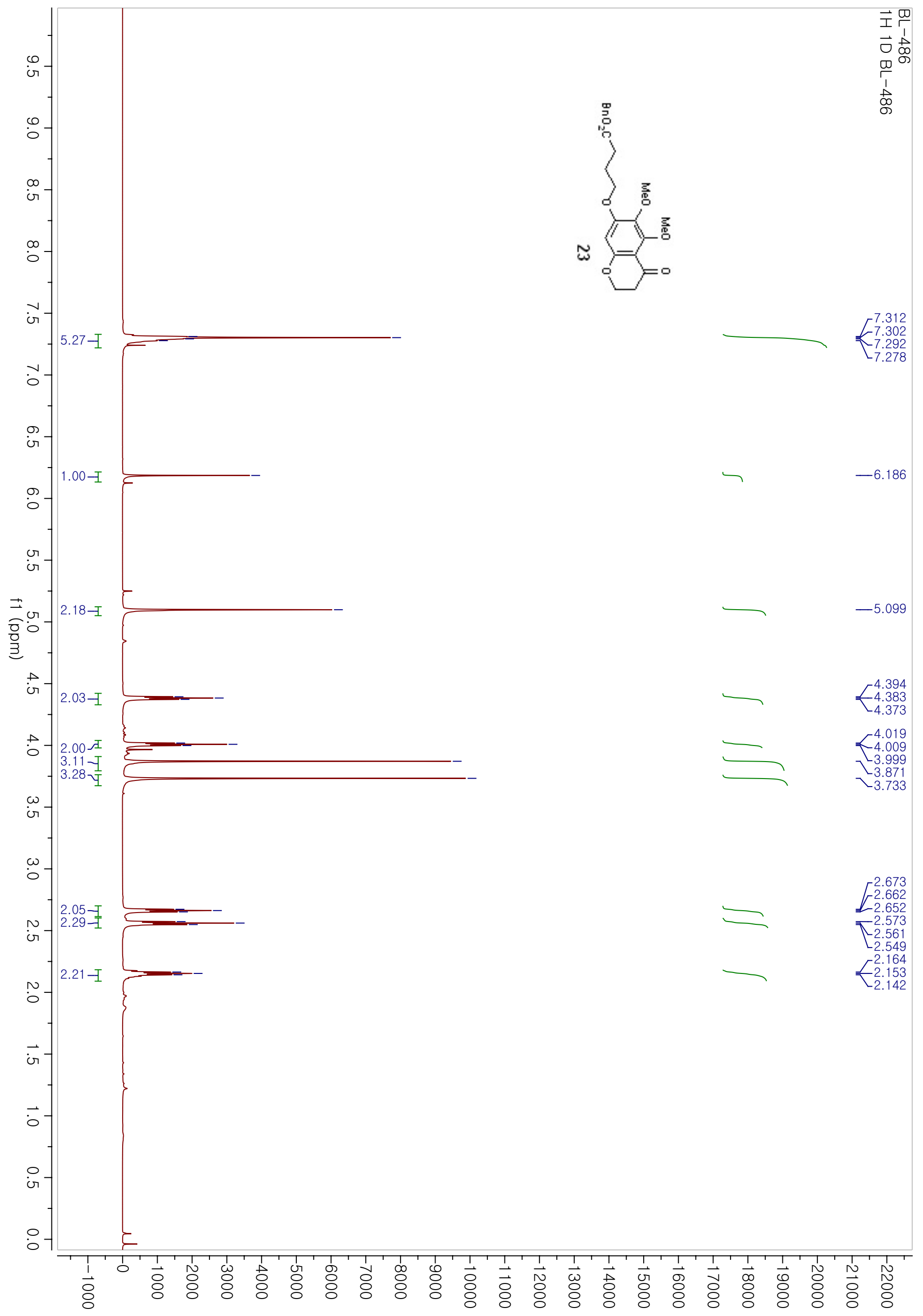




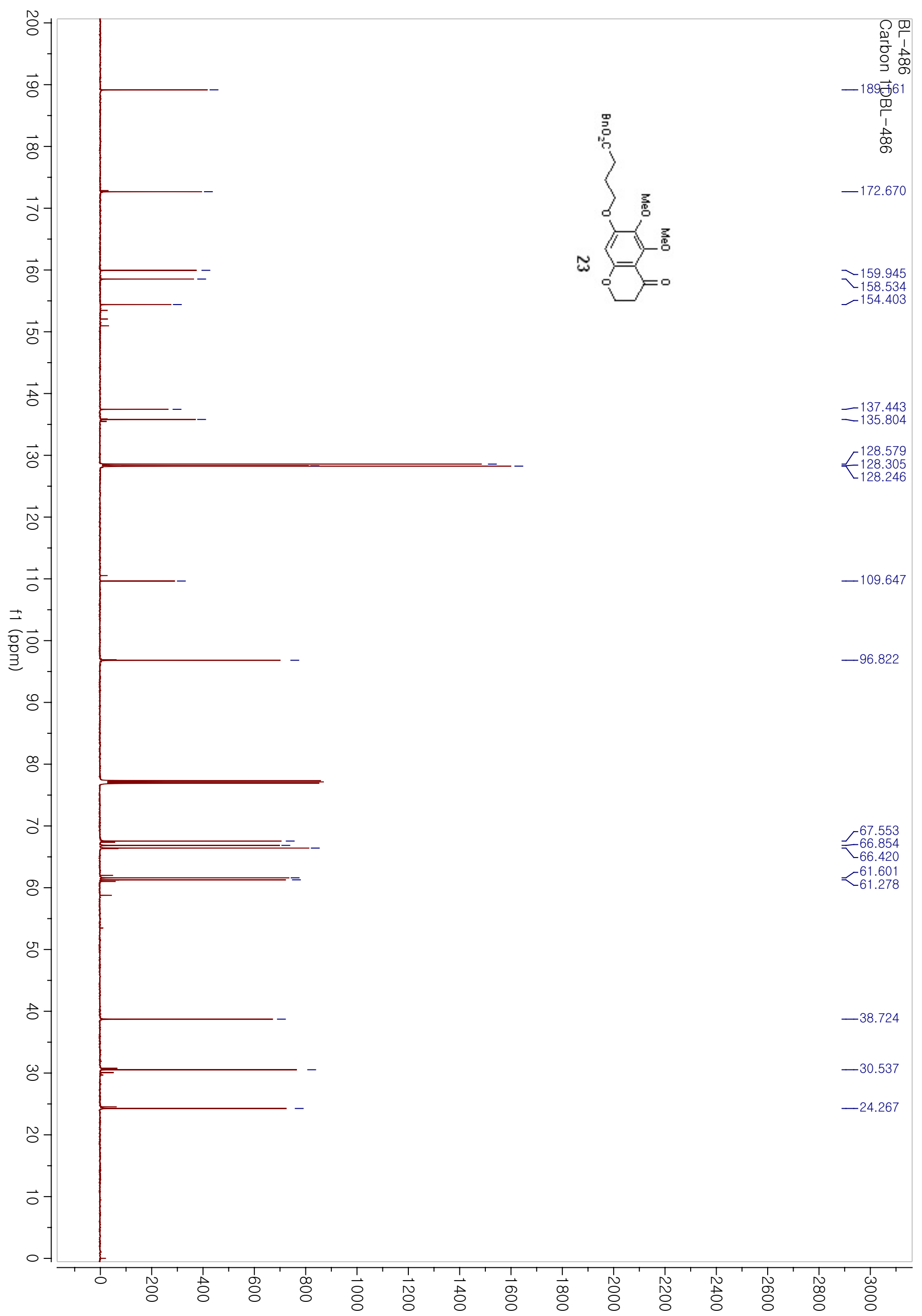




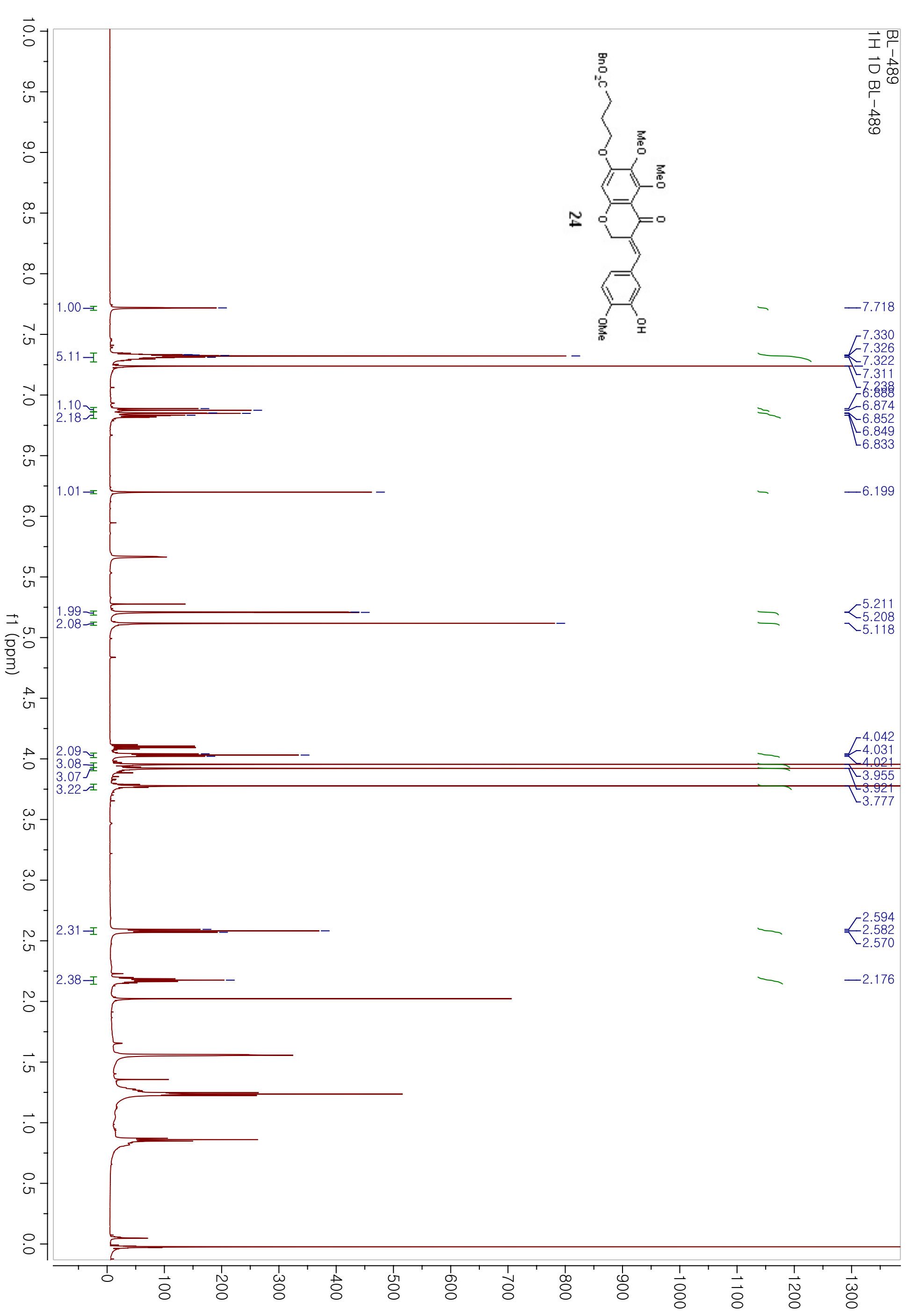



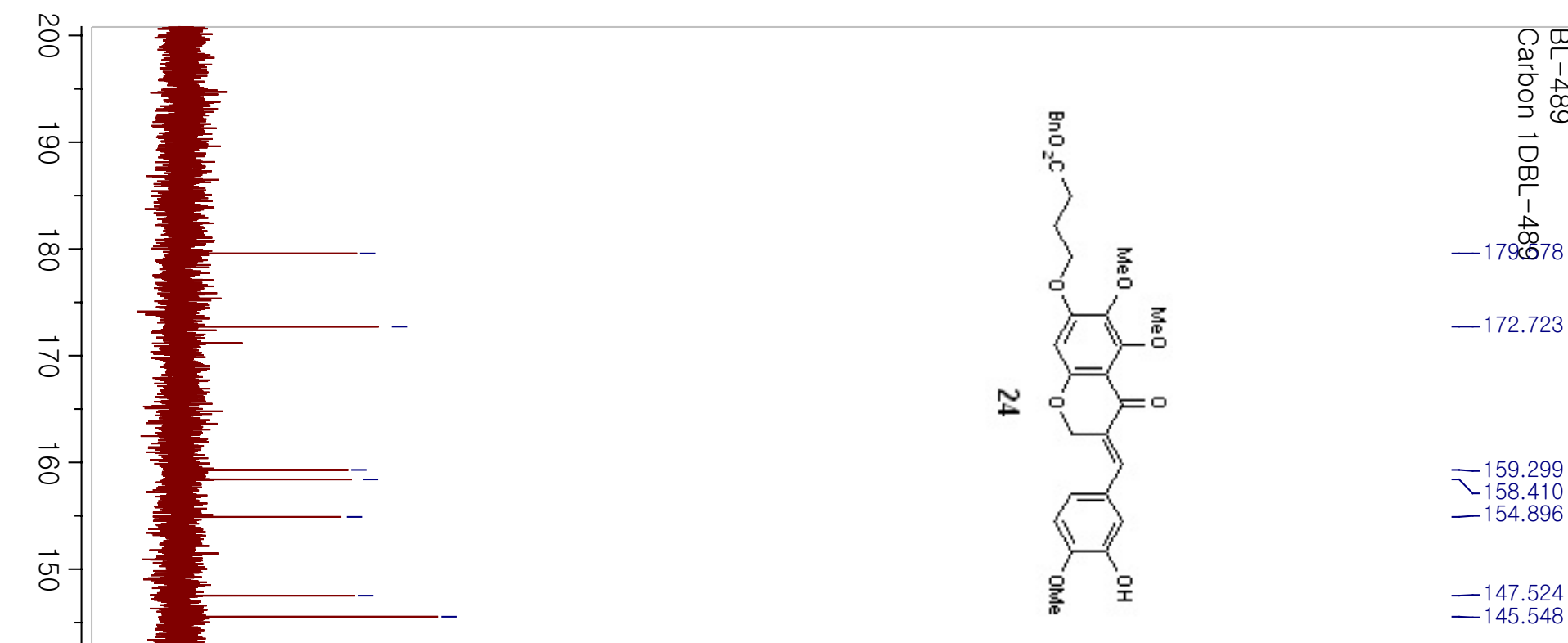

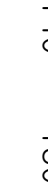

$\overrightarrow{+}$

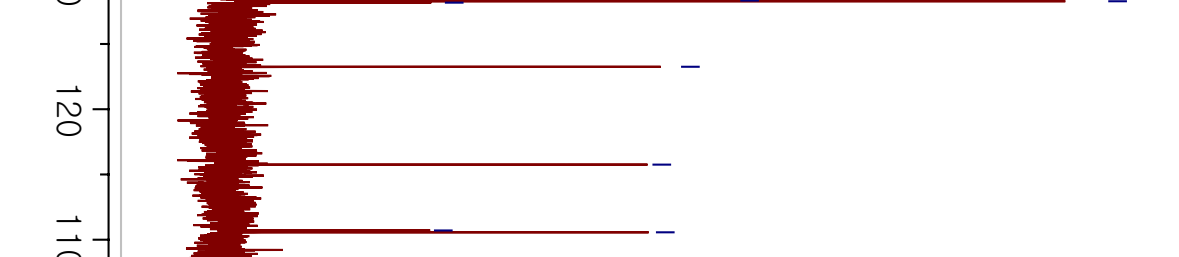

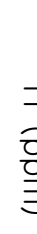

을

$\overrightarrow{0}$

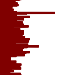

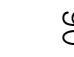

o

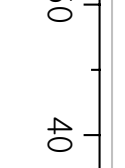

.

$\stackrel{\omega}{\circ}$

กั

$-$

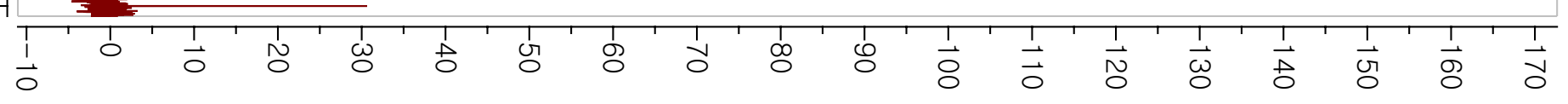




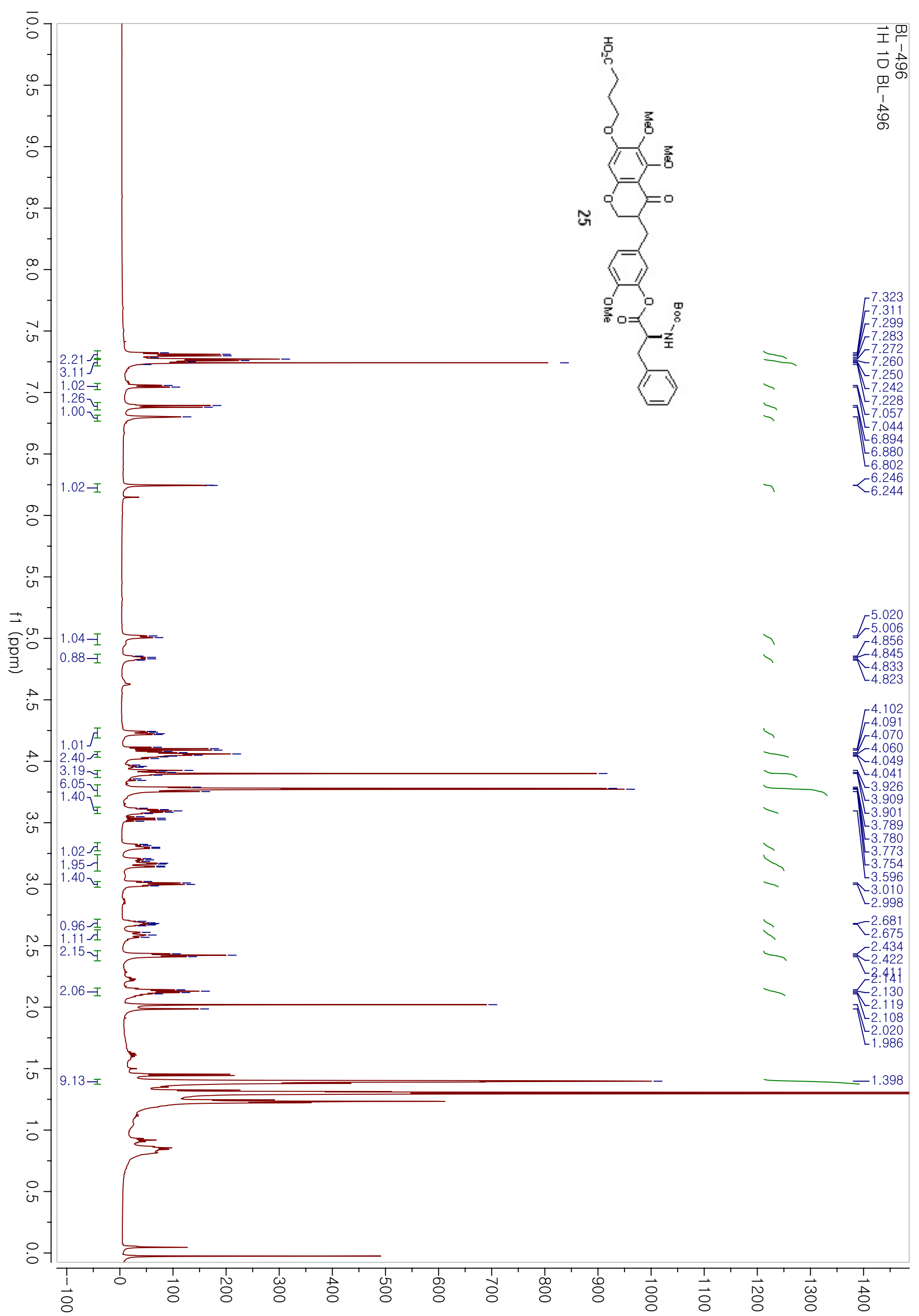




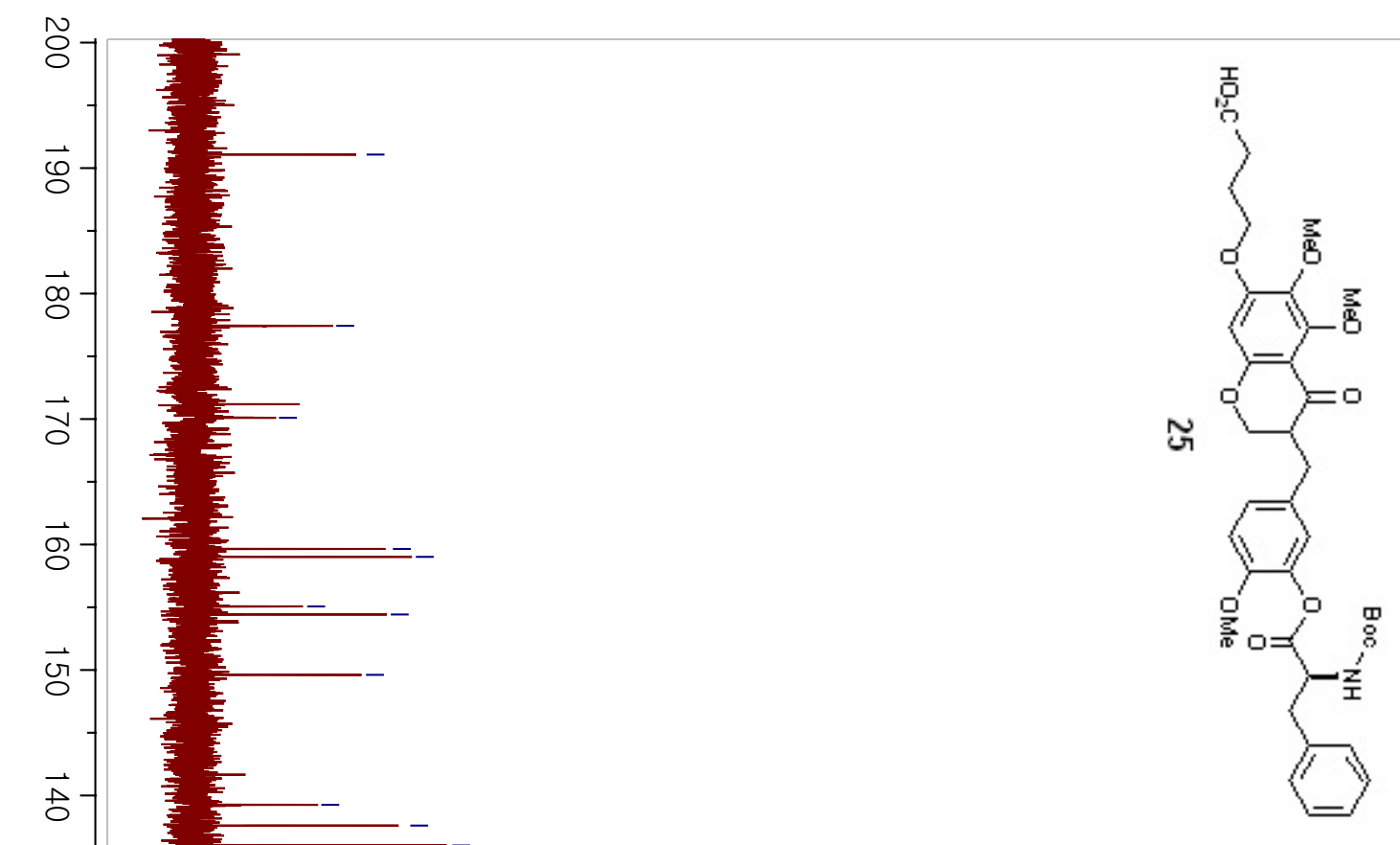

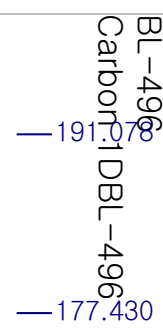

$-170.100$

$-159.647$

$-159.017$

155.068
-154.425

$-149.613$

$-139.257$

$-137.603$

$-136.015$

131.177

$-129.629$

128.550
-127.646

$-127.646$

$\backslash 123.344$

$-112.633$

$-108.420$

웅

$\overrightarrow{0}$

三

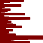

을

궁

$\circ$

- 79.988

$\infty$

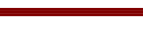

a

$$
8
$$

(1) 列

$\overrightarrow{0}$
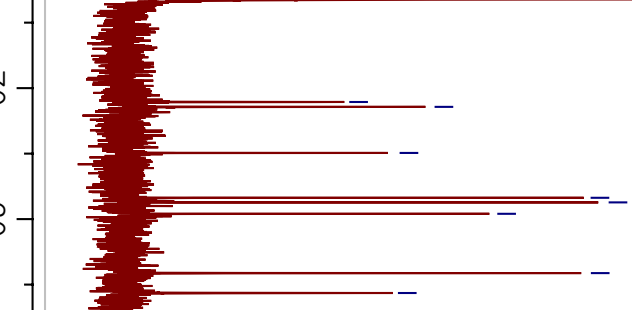

ज-

$-$

-

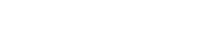

$-68.942$

$-68.570$

$-65.051$

$I_{61.276}^{6.627}$

$-60.405$

$\simeq 55.877$

$\sim 54.357$

$\checkmark 52.079$

$-48.231$

$-40.830$

$-38.289$

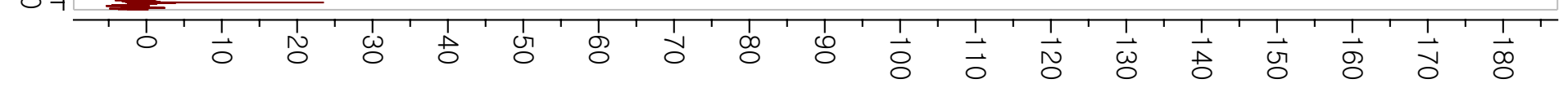




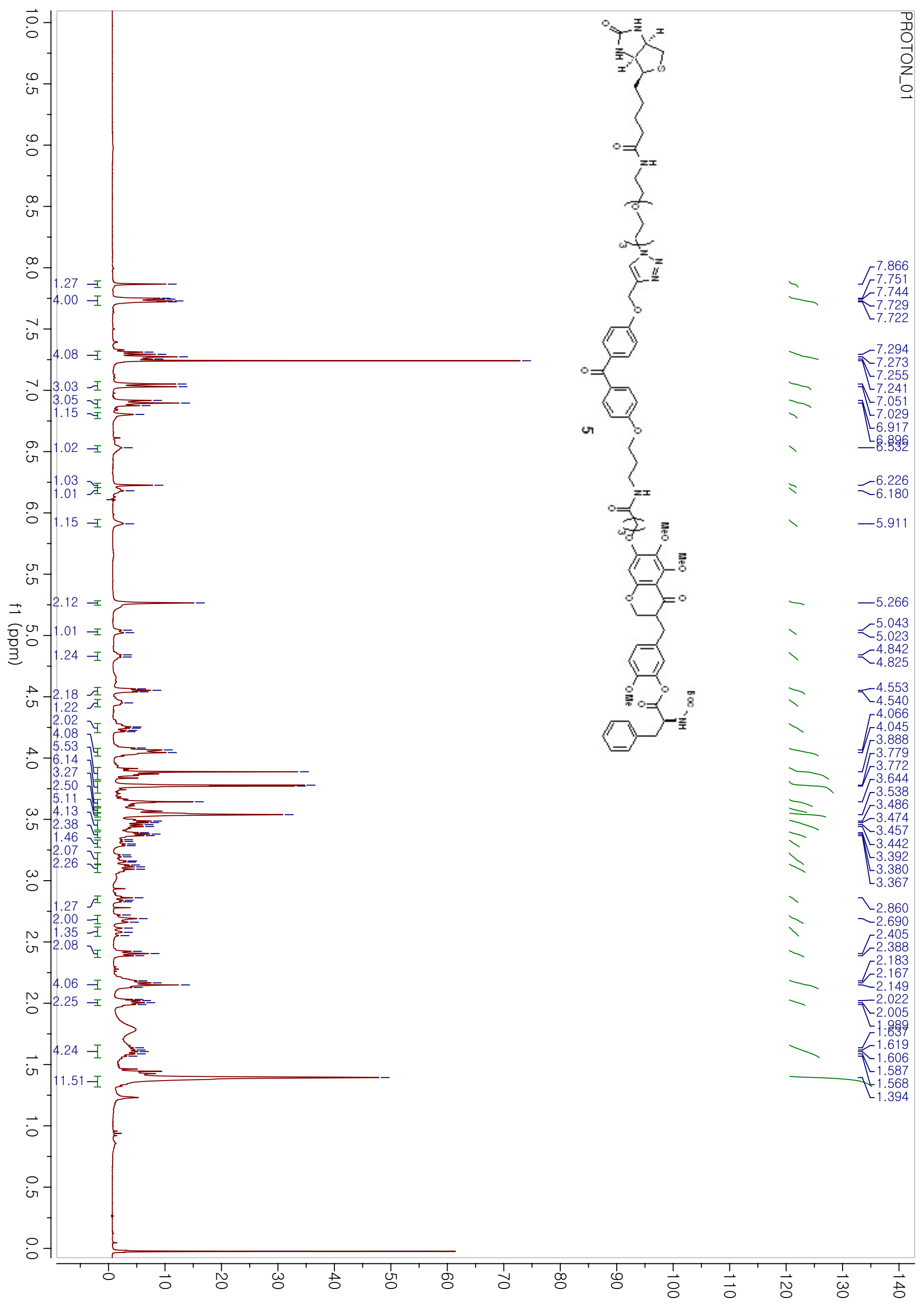




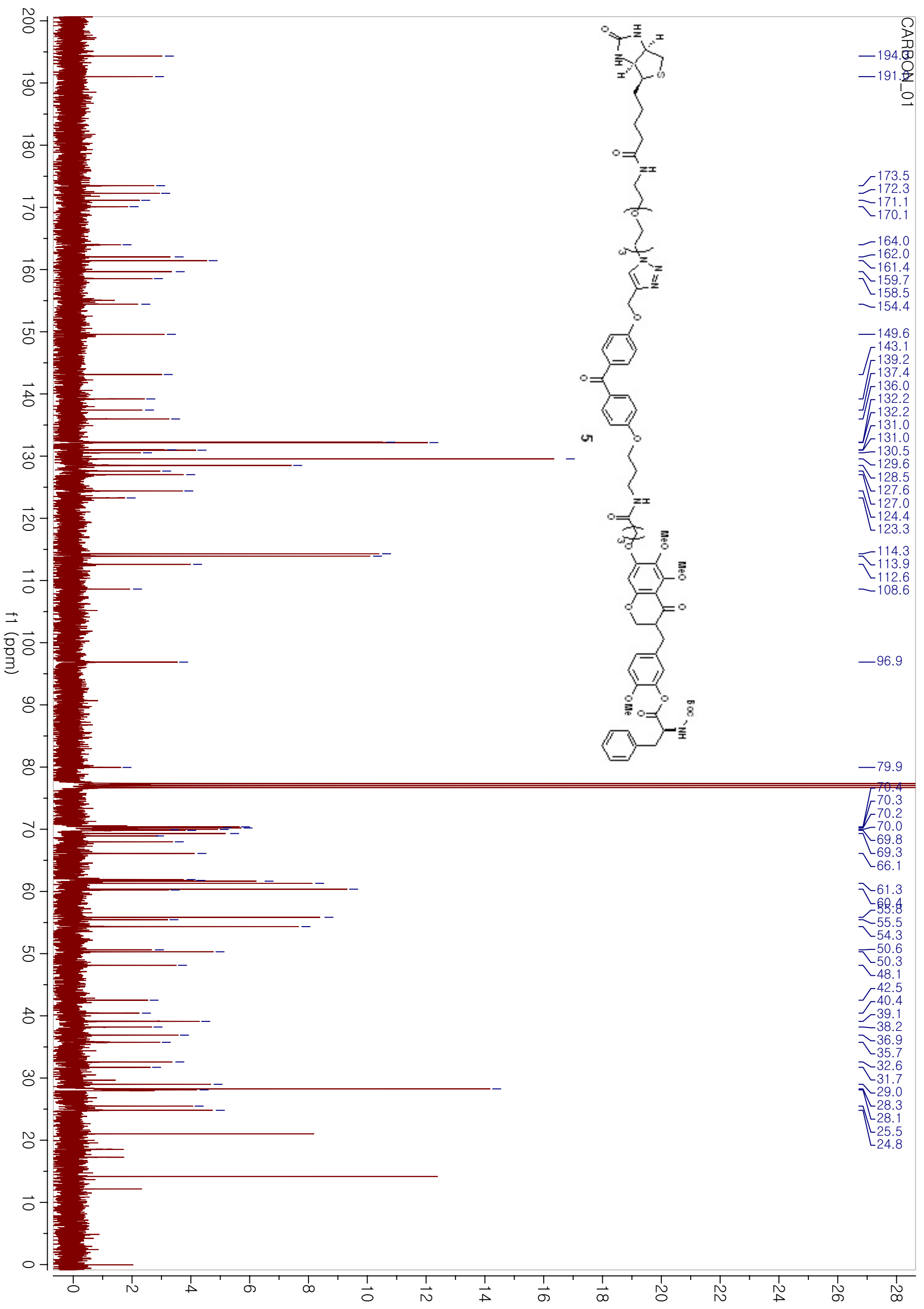

